\title{
An Introduction to Neurotechnologies, Transcranial Magnetic Stimulation and Transcranial Direct Current Stimulation: Their Applications in the Cognitive Enhancement and Rehabilitation
}

\author{
Mohammad Javad Eslamizade $^{1,2}$, Shahram Behbahanian ${ }^{3}$, Seyed Mohammad Mahdavi ${ }^{4}$, Mohsen Oftadehal ${ }^{3 *}$ \\ ${ }^{1}$ Department of Neuroscience, Faculty of Advanced Technologies in Medicine, Iran University of Medical Sciences, Tehran, Iran \\ ${ }^{2}$ Shefa Neuroscience Research Center, Khatam Alanbia Hospital, Tehran, Iran \\ ${ }^{3}$ Institute for Cognitive Science Studies, Institute Boulevard, Pardis City, Tehran, Iran \\ ${ }^{4}$ Department of Medical Physics, Malek-Ashtar University of Technology, Tehran, Iran
}

\section{A BSTRACT}

Introduction: Non-invasive brain stimulation technologies are a group of powerful tools that are exploited in manipulating functional properties of the brain. These technologies have a relatively long history in cognitive enhancement and rehabilitation, as well as in treating stress and depression. Of these technologies are transcranial magnetic stimulation (TMS) and transcranial direct current stimulation (tDCS). TMS technology works with producing magnetic fields emitted from a coil over the scalp to induce ion currents; however, in tDCS there is a direct connection of electrodes on the skin over the scalp to inject electrical currents yielding modulation in neural circuits. Conclusion: In this review, we reviewed over the literature concerning applications of neurotechnologies, TMS and tDCS, in cognitive enhancement and rehabilitation.

*Corresponding Author: Mohsen Oftadehal

E-mail:dr.oftadeh@gmail.com 


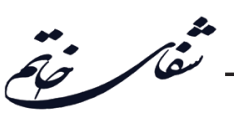

معرفى فناورى هاى تحريك مغناطيسى وراجمجمهاى و تحريك الكتريكى وراجمجمهاى: كاربردهاى آنها در ارتقاء عملكردهاى شناختى و باز توانى وريسى شناختى

\section{محمد جواد اسلامى زاده־'، شهرام بجببهانيان"، سيد محمد مهدوى"، محسن افتاده حال ״"}

'ئروه علوم اعصاب، دانشكده فن آورىهاى نوين يزشكى، دانشعاه علوم يزشكى ايران، تهران، ايران

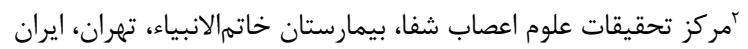

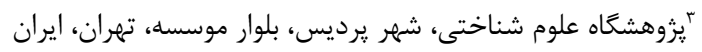

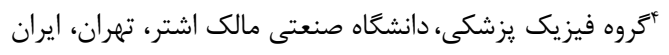

كليد وازهها:

إخ

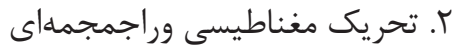

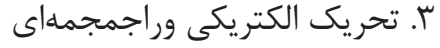

مقدمه: فناورىهاى تحريك كننده غيرتهاجمى مغز، ابزارى قدرتمند در تحريك و دستكارى

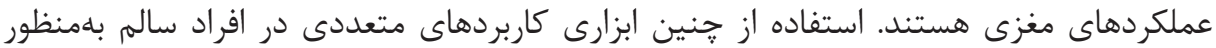

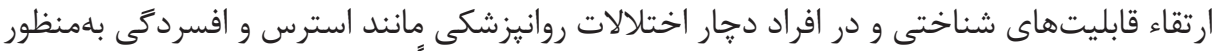

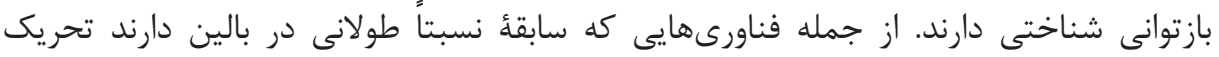

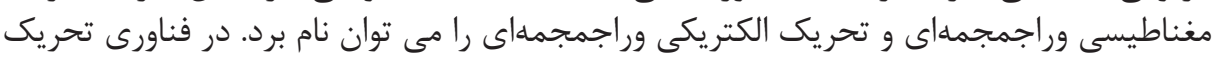

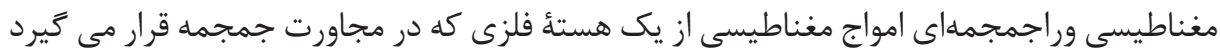

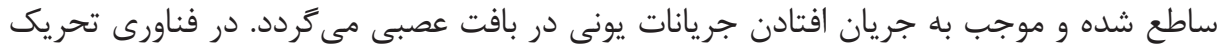

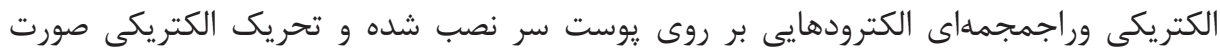

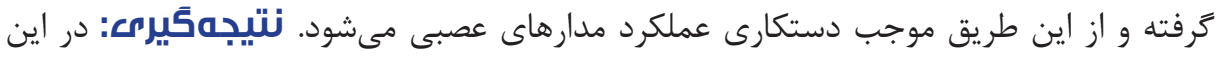

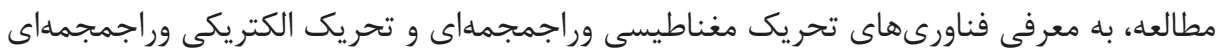

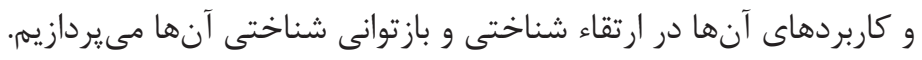

"نويسنده مسئول: محسن افتاده حال آدرس الكترونيكى: dr.oftadeh@gmail.com 


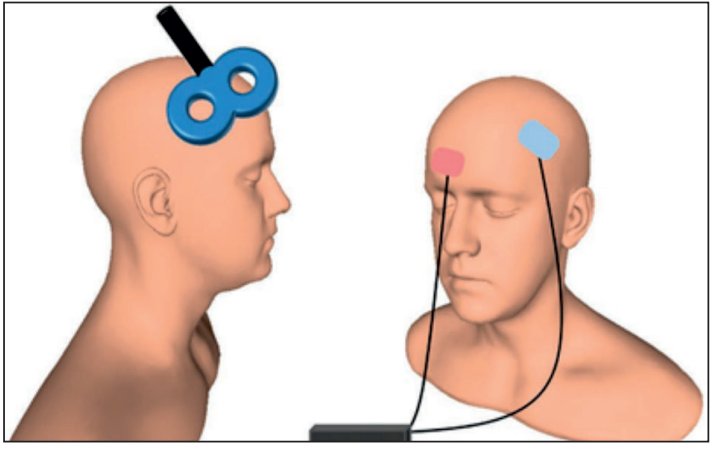

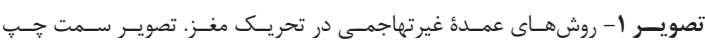

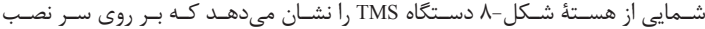

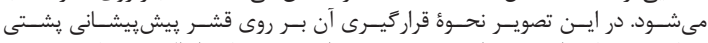

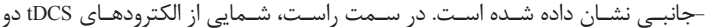

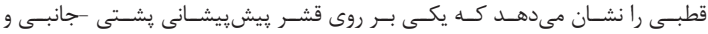

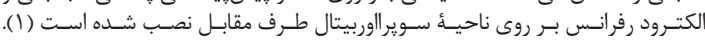

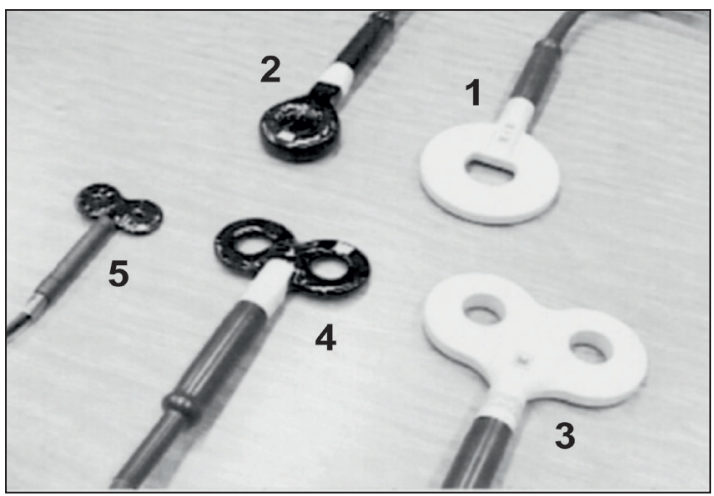

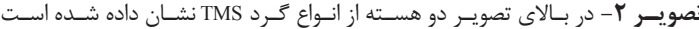

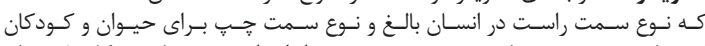

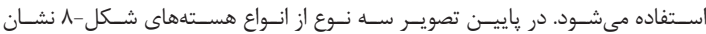

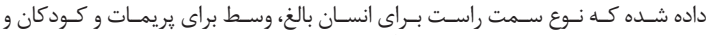

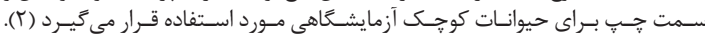

مقدمهاى بر TMS و بررسى مكانيسمهاى آن فنــاورى TMS در ســال 1919 معرفـى شــد و از آن ســال

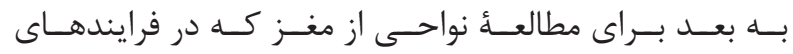

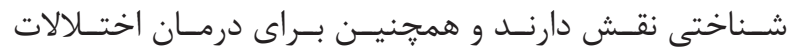

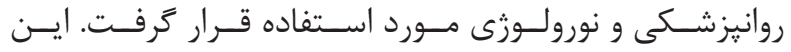

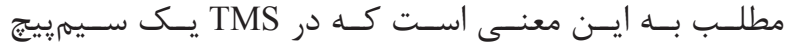

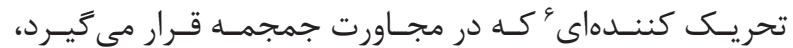

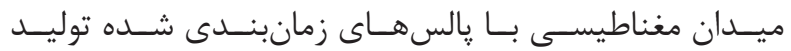

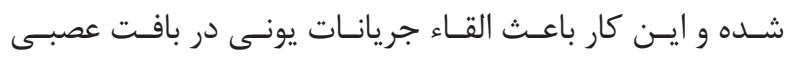

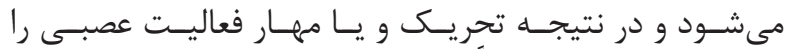

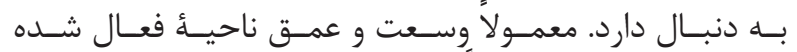

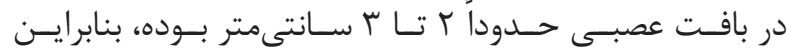

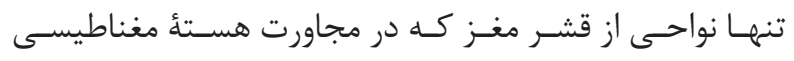

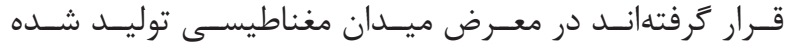

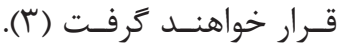

${ }^{1}$ Cognitive functions

${ }^{2}$ Cognitive neuroscience

${ }^{3}$ Awareness

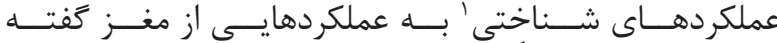

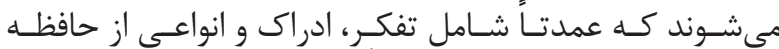

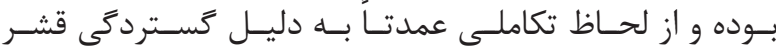

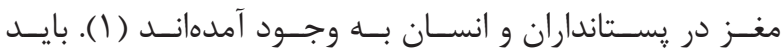

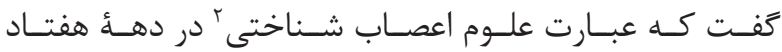

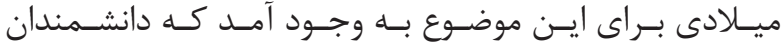

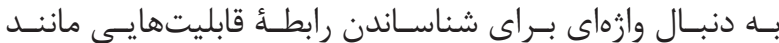

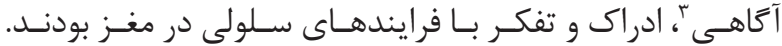

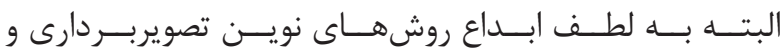

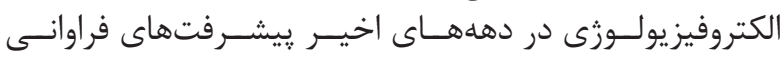

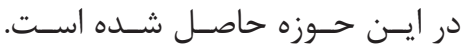

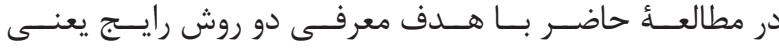

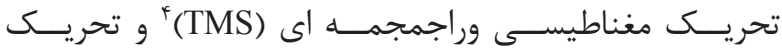

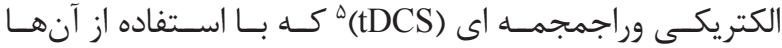

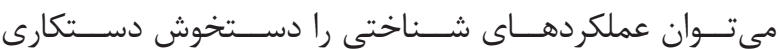

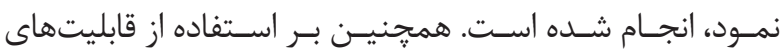

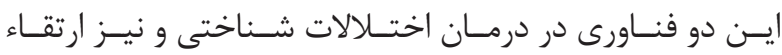

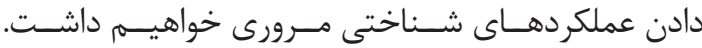
دستكارى عملكردهاى مغزى با استفاده از امواج

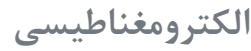

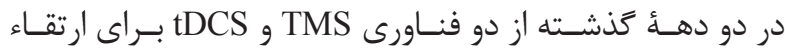

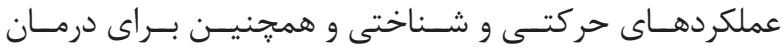

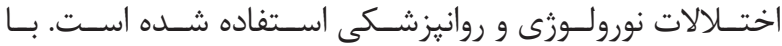

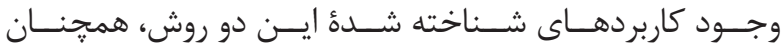

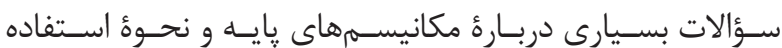

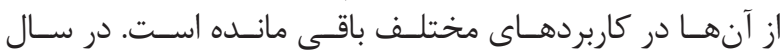

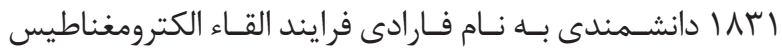

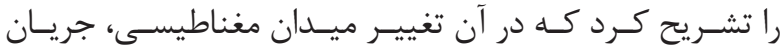

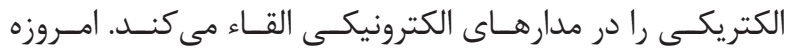

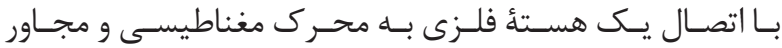

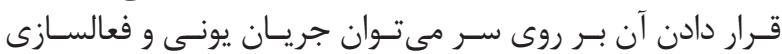

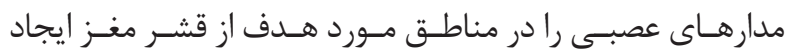

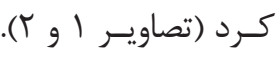

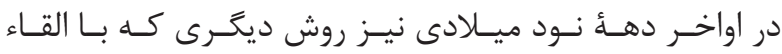

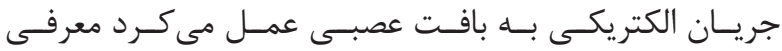

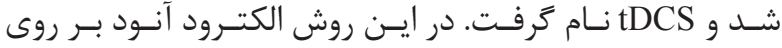

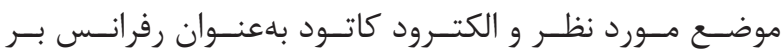

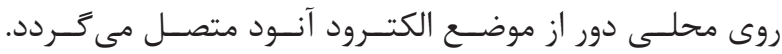

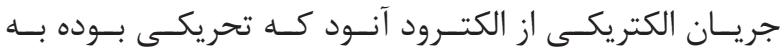

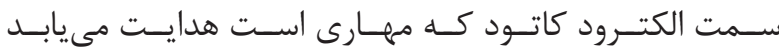

(1) (1) (1) (1) (1) (1) 


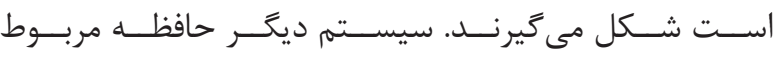

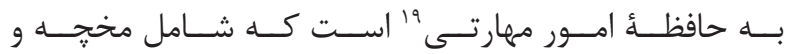

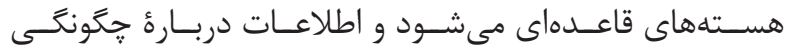

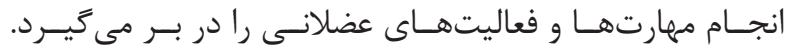

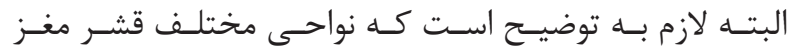

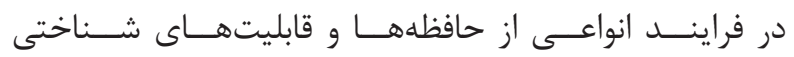

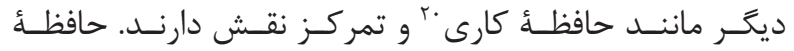

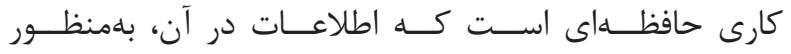

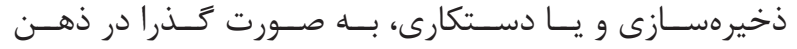

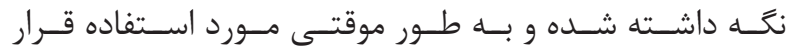

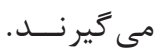
استفاده از TMS در بررسى نقش قشر يِيشيشيشانى در تداخل حافظهها

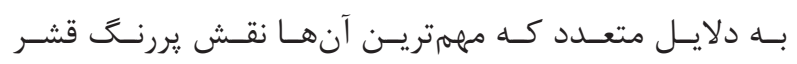

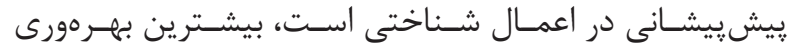

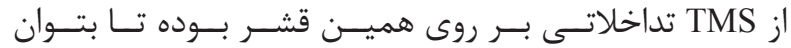

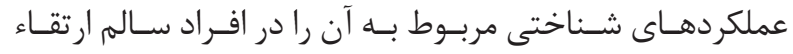

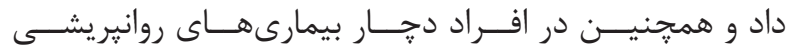

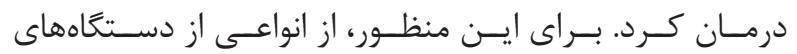
TMS

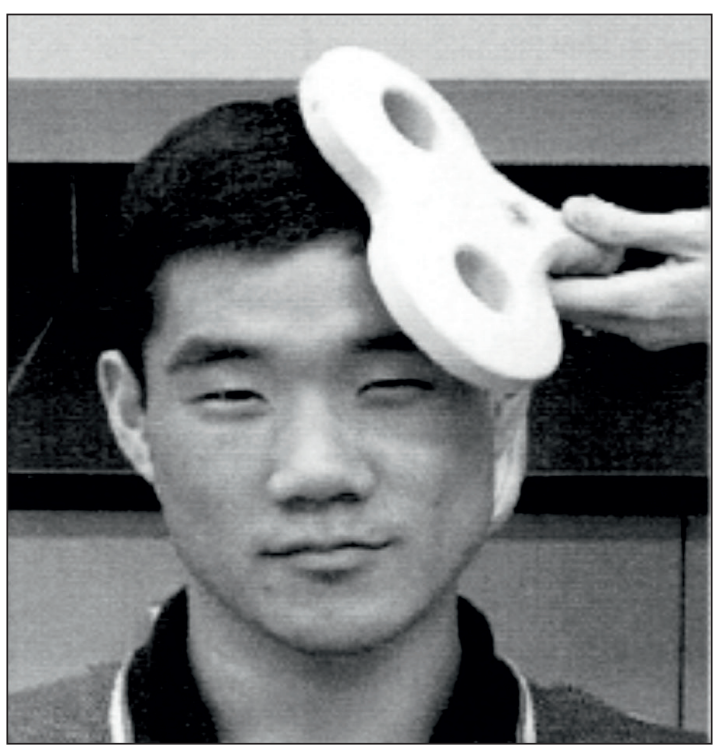

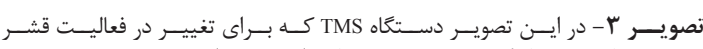

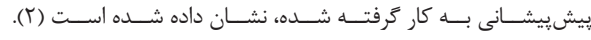

عـلاوه بـر اسـتفاده از TMS در مداخلـه و درمــان بيمارىهـا،

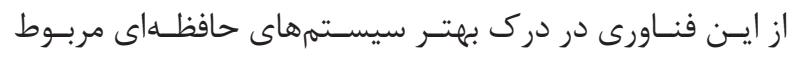

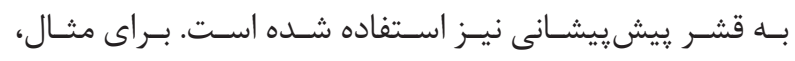

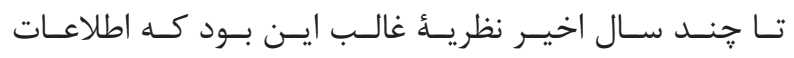

${ }^{7}$ Motor cortex

${ }^{8}$ Frontal cortex

${ }^{9}$ Positron emission tomography

${ }^{10}$ Neurotransmission

${ }^{11}$ Subcortical areas

${ }^{12}$ Medial prefrontal areas

${ }^{13}$ Motor threshold

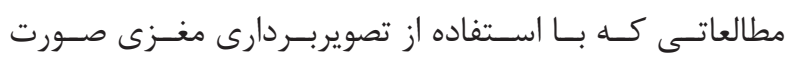

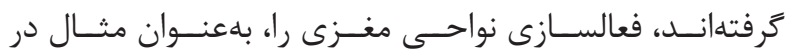

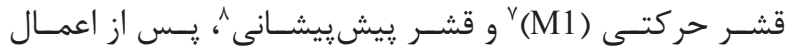

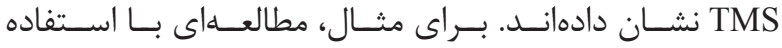

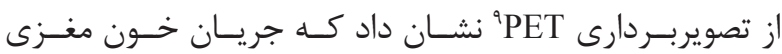

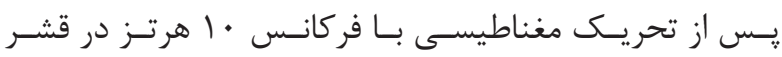

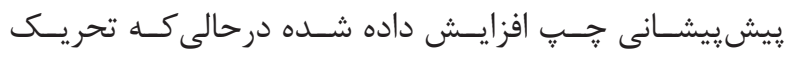

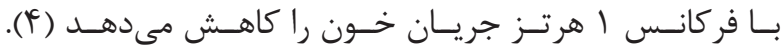

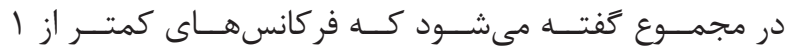

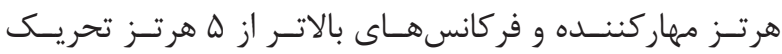

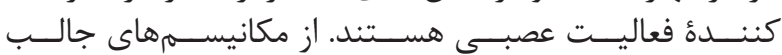

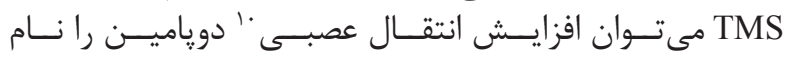

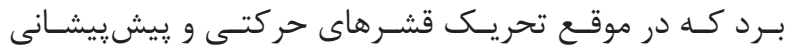

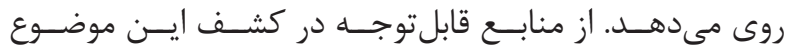

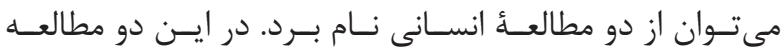

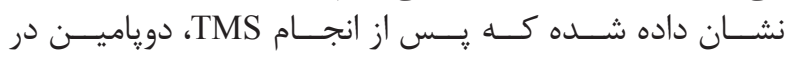

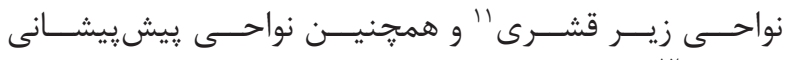

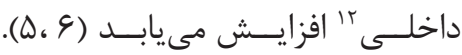

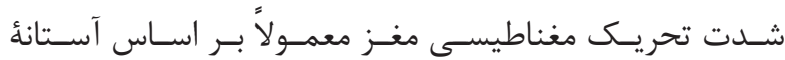

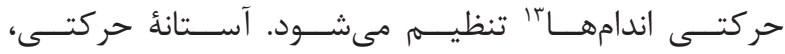

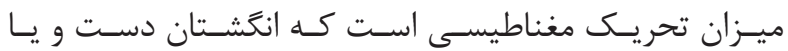

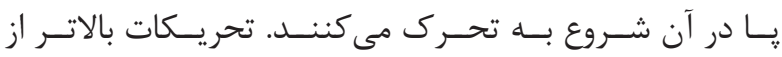

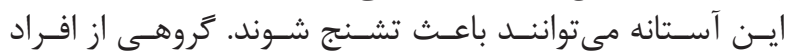

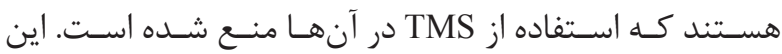

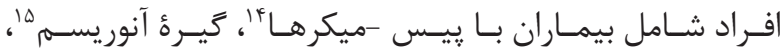

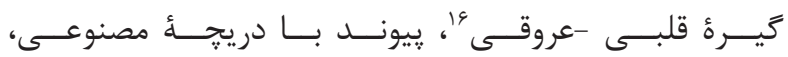

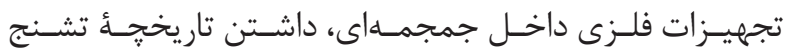

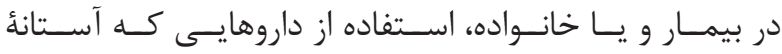

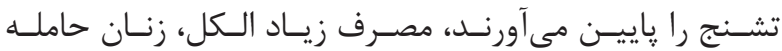

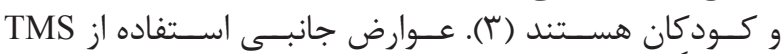

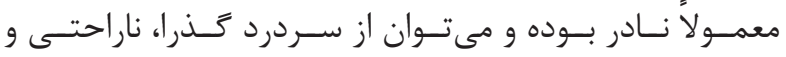

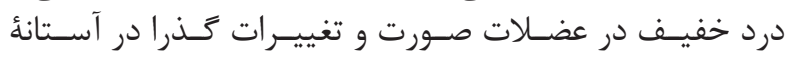

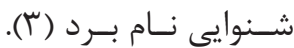
استفاده از TMS براى ارتقاء حافظه در ايسن قسـمت، نخسـت لازم اسـت توضيـح داده شـود كــه

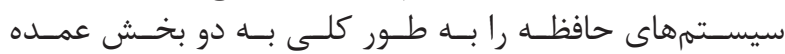

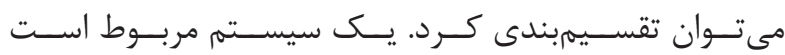

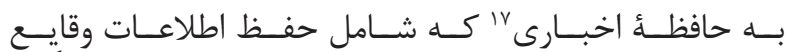

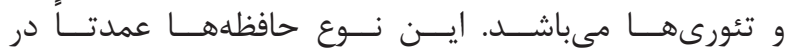

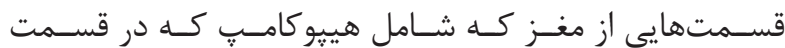

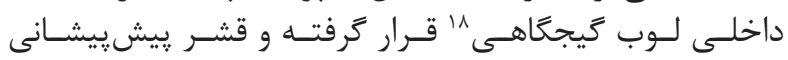

${ }^{14}$ Pacemakers

${ }^{15}$ Aneurysm clip

${ }^{16}$ Heart-vascular clip

${ }^{17}$ Declarative memory

${ }^{18}$ Temporal lobe

${ }^{19}$ Procedural memory

${ }^{20}$ Working memory 


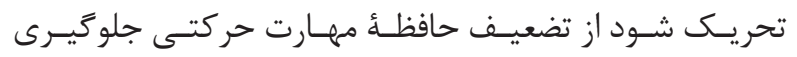

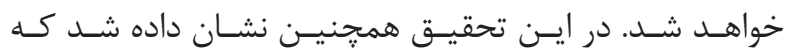

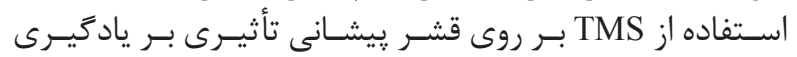

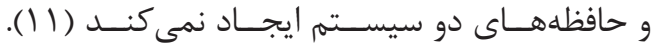

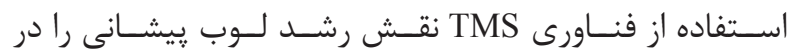

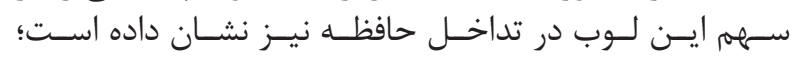

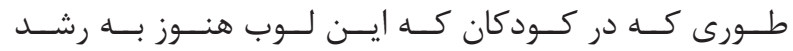

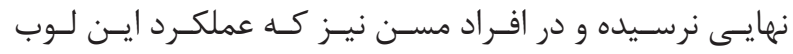

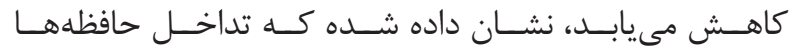

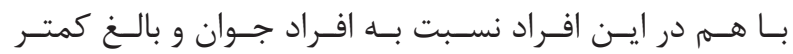

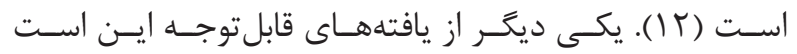

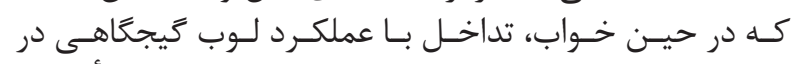

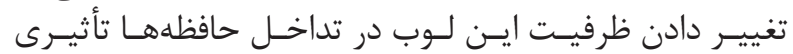

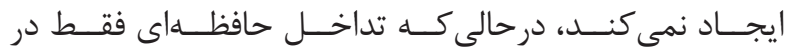

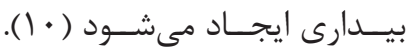

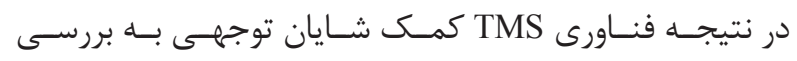

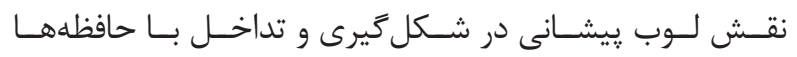

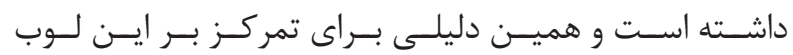

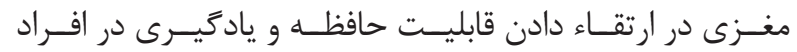

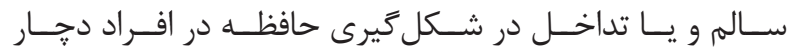

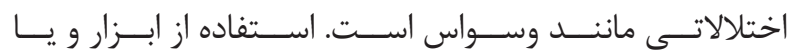

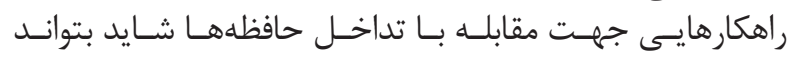

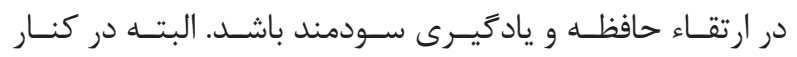

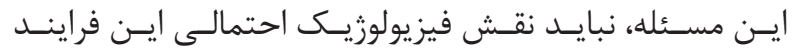

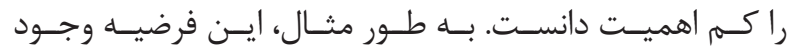

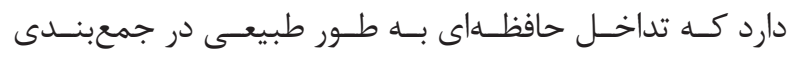

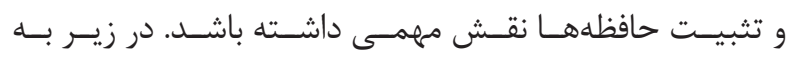

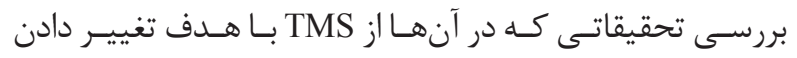

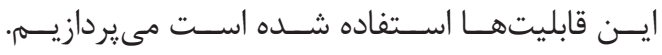

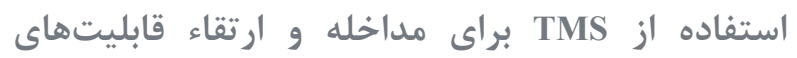
شناختى مربوط به قشر يِيش ييشانى

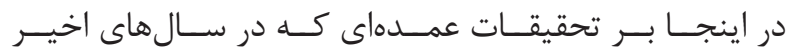

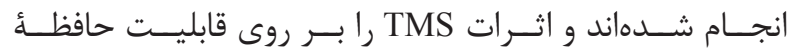

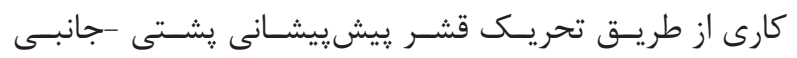

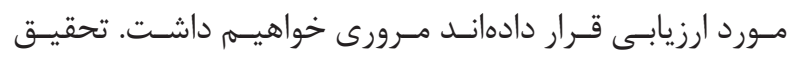

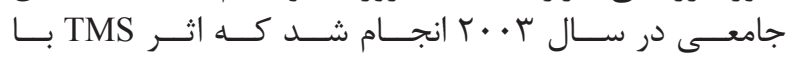

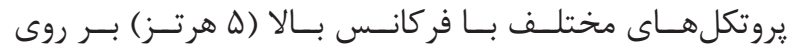

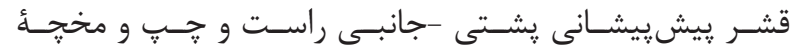

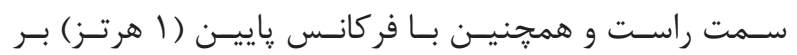

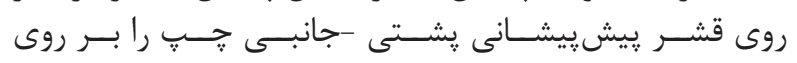

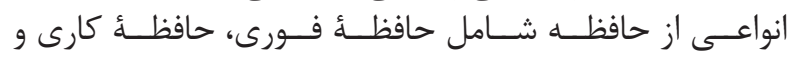

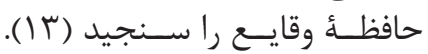

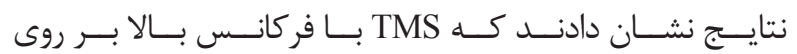

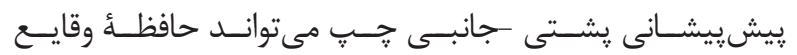

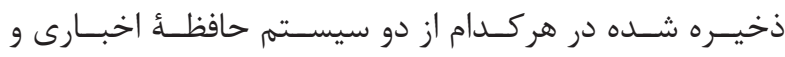

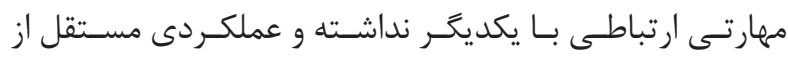

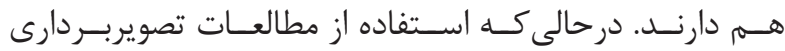

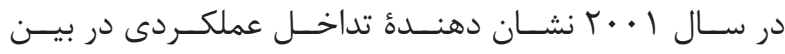

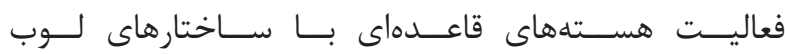

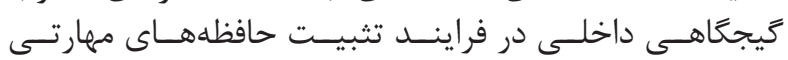

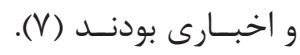

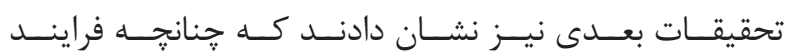

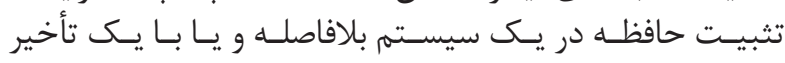

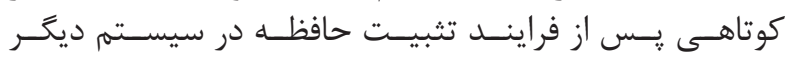

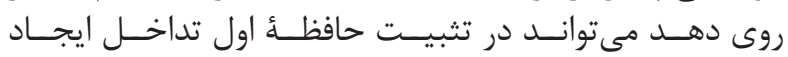

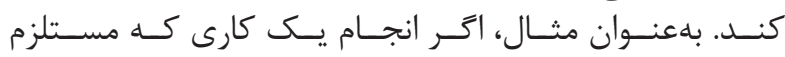

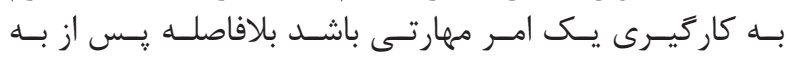

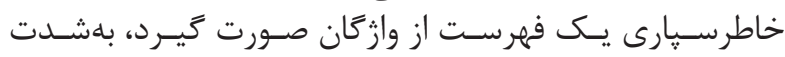

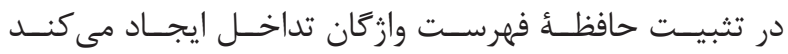

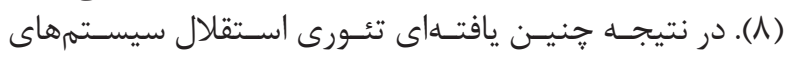

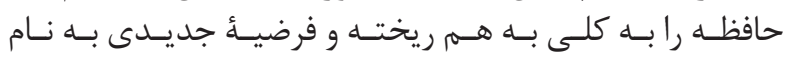

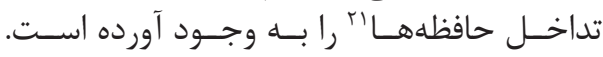

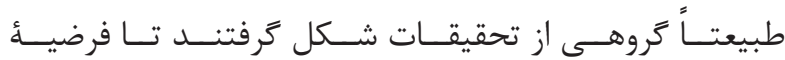

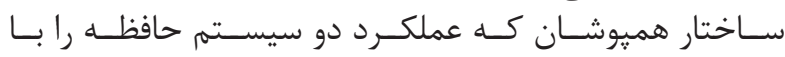

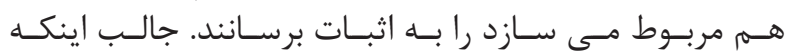

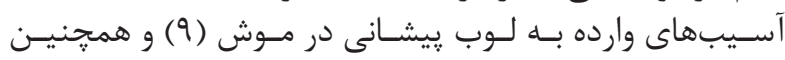

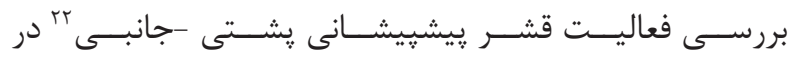

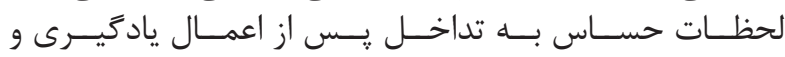

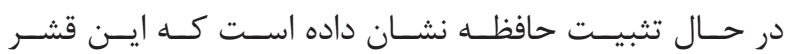

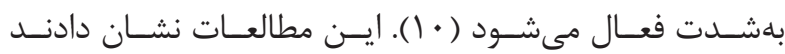

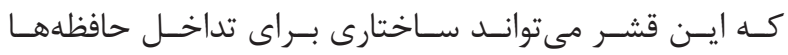

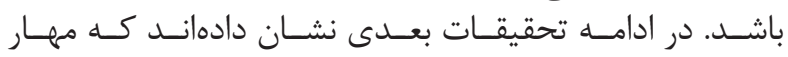

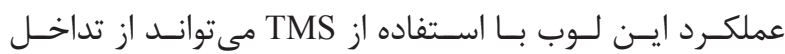

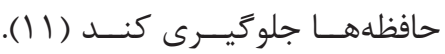

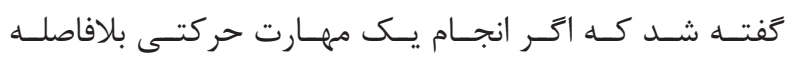

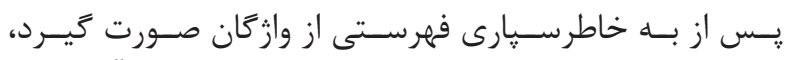

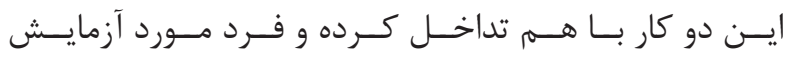

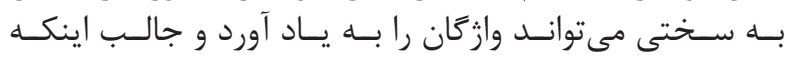

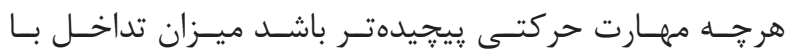

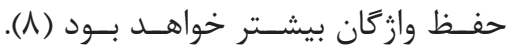

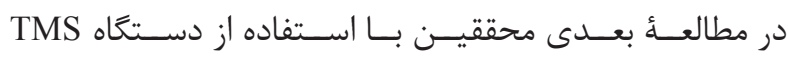

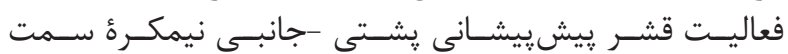

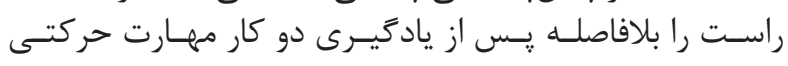

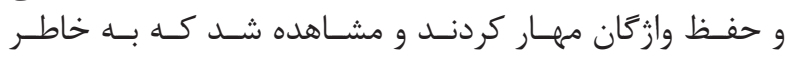

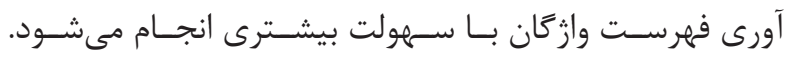

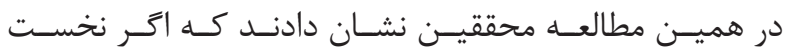

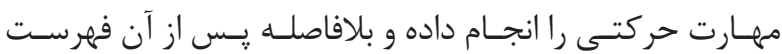

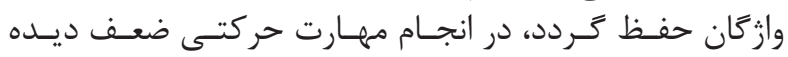

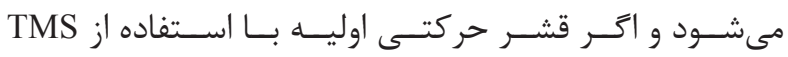


يـردازش اطلاعـات وارده بـهـ سيسـتم عصبـى ايجــاد مى كنــد.

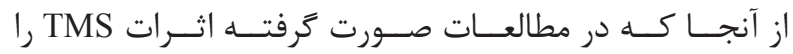

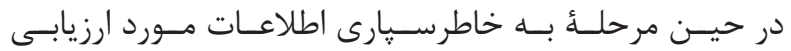

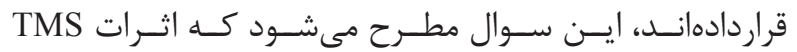

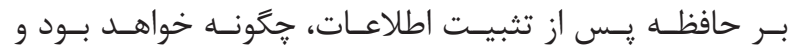

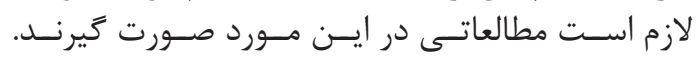
استفاده از TMS بر اى ار تقاء حافظةٌ كارى مربوط به قشر

بس

اطلاعـات جالـب توجهـى از عملكـرد قشــر بينايسى واقـع در

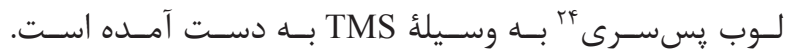

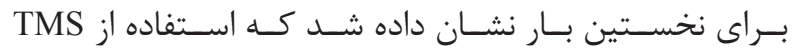

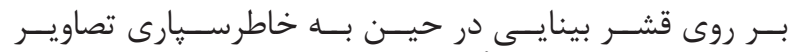

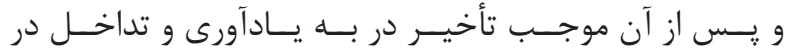

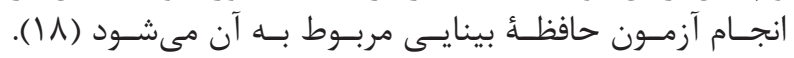

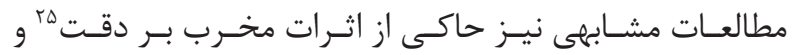

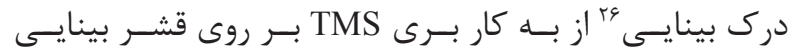

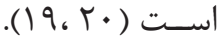

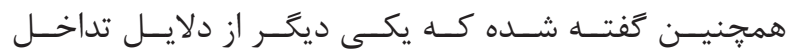

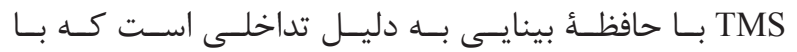

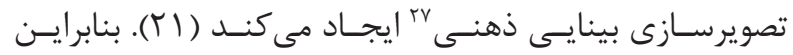

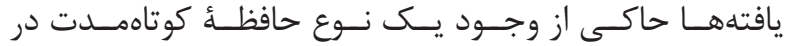

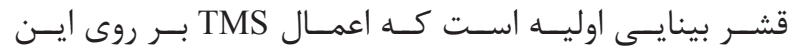

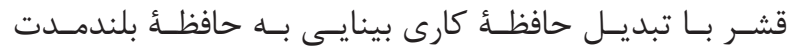

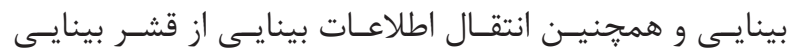

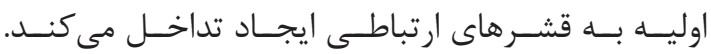

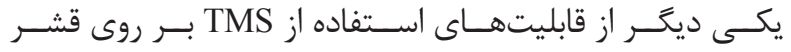

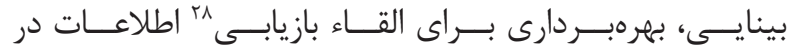

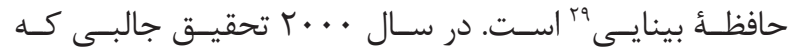

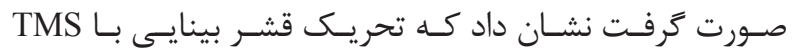

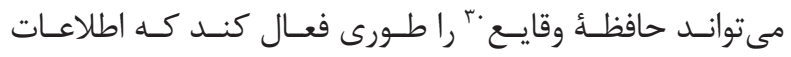

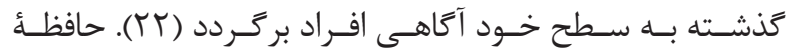

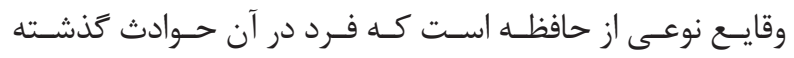

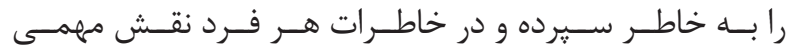

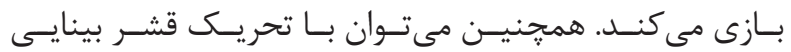

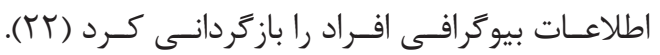
استفاده از TMS براى ارتقاء قابليتهاى شناختى مربوط به قشر كَيجًاهى

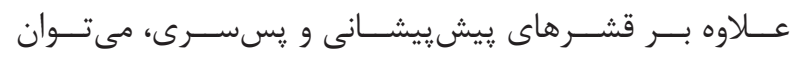

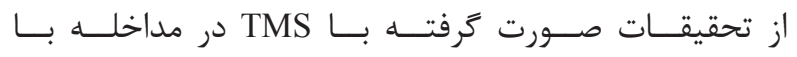

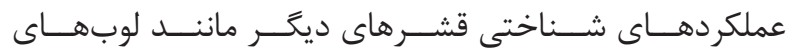

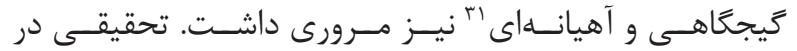

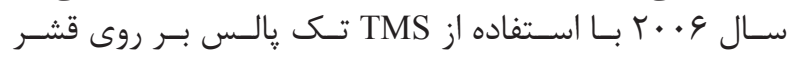

${ }^{23}$ Repetitive TMS

${ }^{24}$ Occipital lobe

${ }^{25}$ Visual accuracy

${ }^{26}$ Visual perception

${ }^{27}$ Visual mental imagery

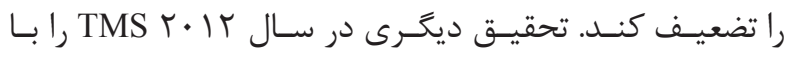

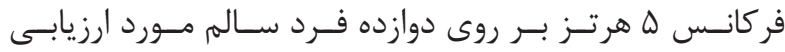

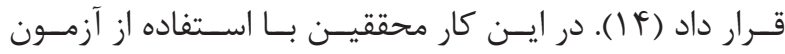

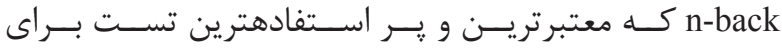

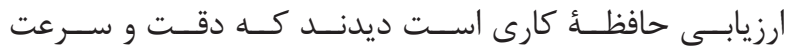

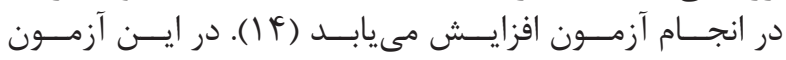

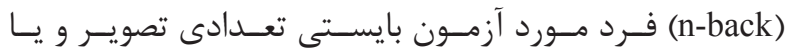

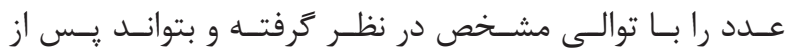

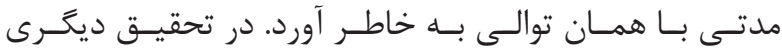

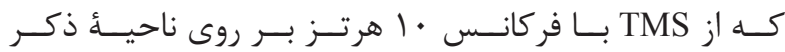

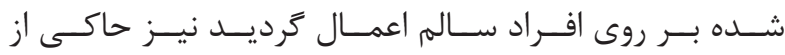

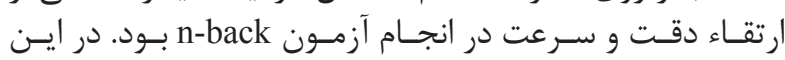

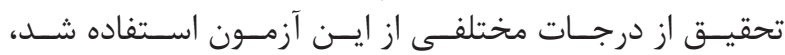

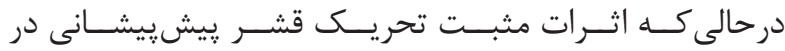

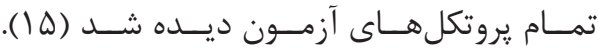

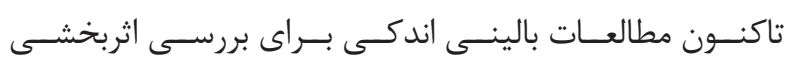

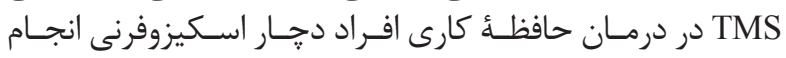

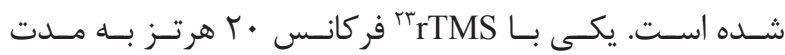

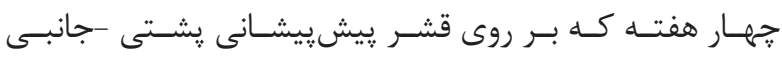

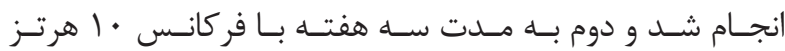

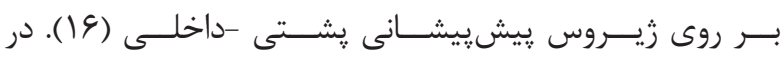

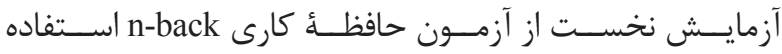

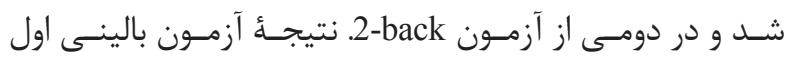

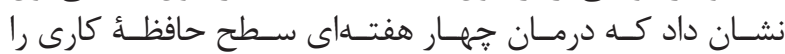

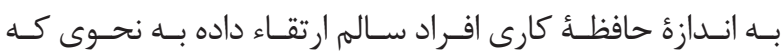

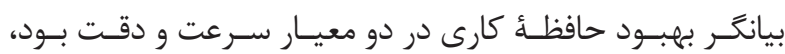

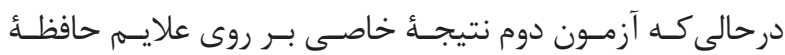

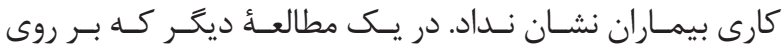

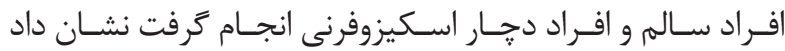

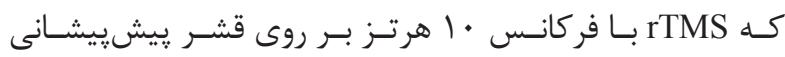

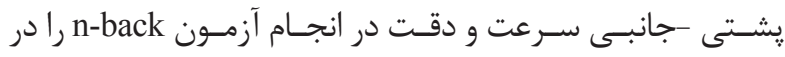

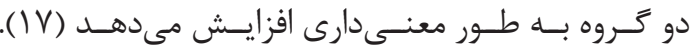

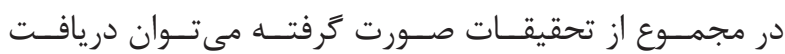

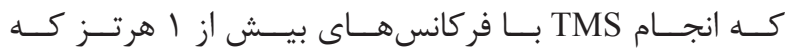

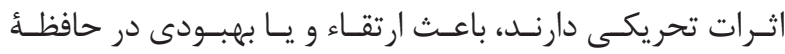

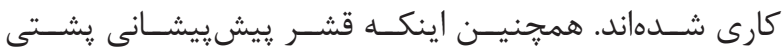

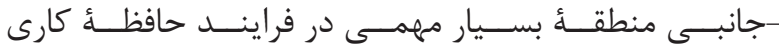

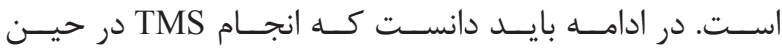

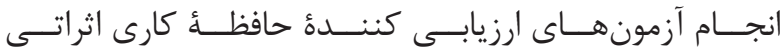

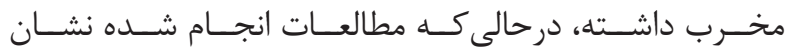

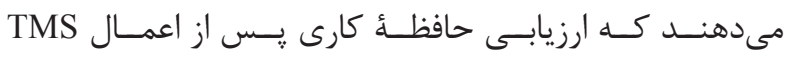

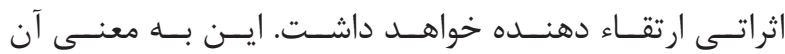

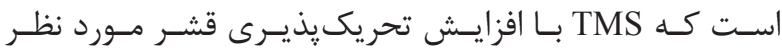

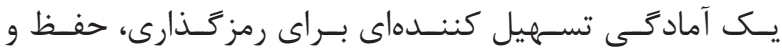

\footnotetext{
${ }^{28}$ Reactivation

${ }^{29}$ Visual memory representations

${ }^{30}$ Episodic memory

${ }^{31}$ Parietal lobe
} 


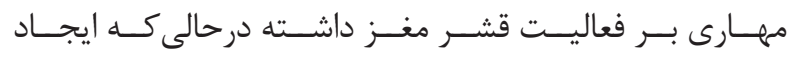

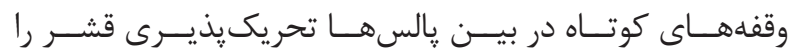

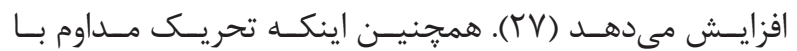

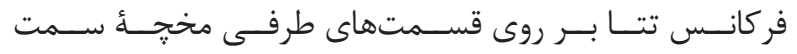

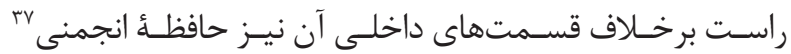

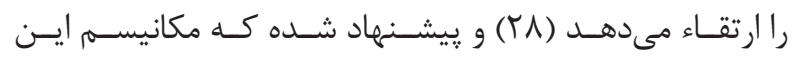

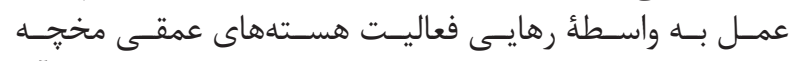

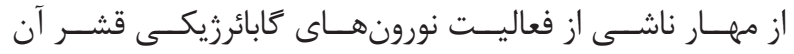

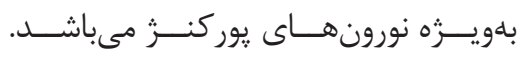

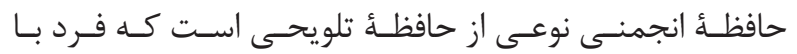

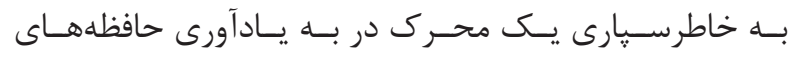

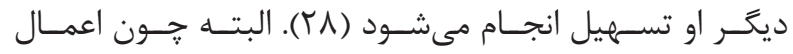
TMS

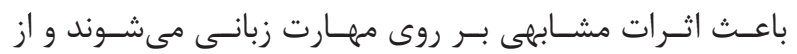

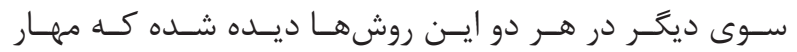

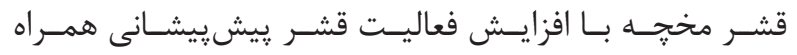

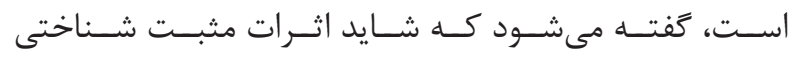

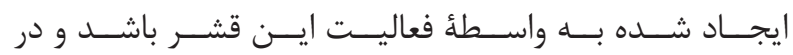

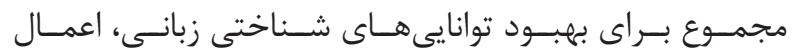

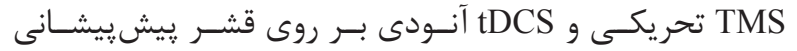
يشـتى -جانبـى سـمت جـــ

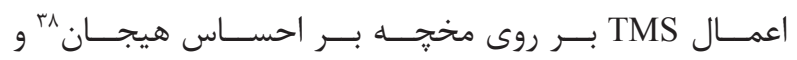

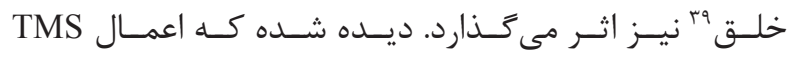

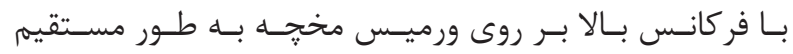

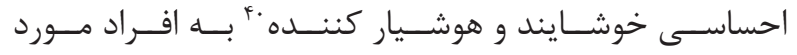

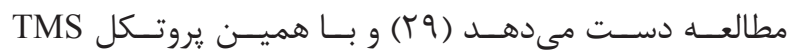

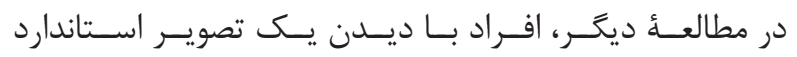

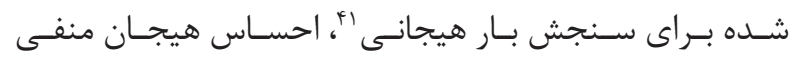

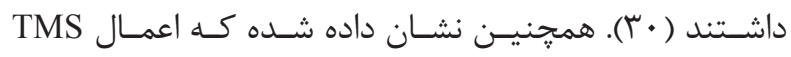

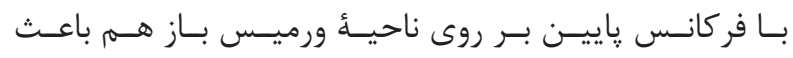

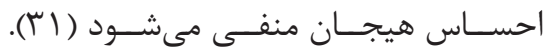

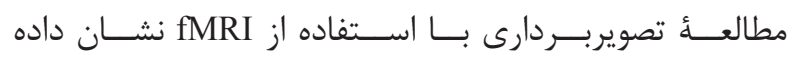

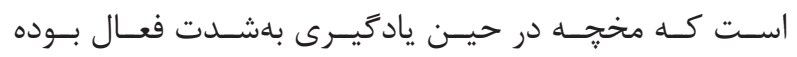

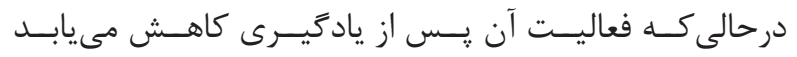

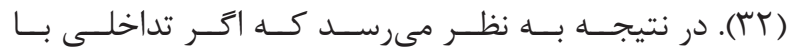

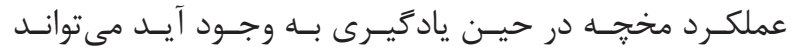

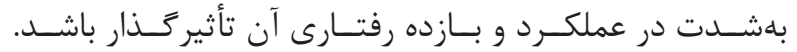

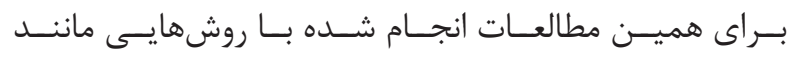

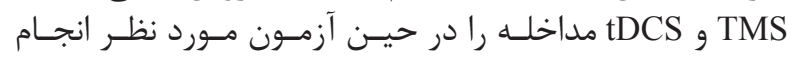

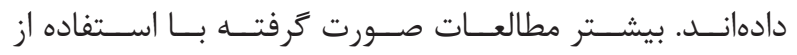

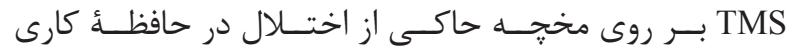

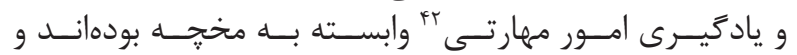

\footnotetext{
${ }^{32}$ Verbal processing

${ }^{33}$ Phonemic and semantic fluency

${ }^{34}$ Lexical decisions

${ }^{35}$ Continuous theta frequency stimulation

${ }^{36}$ Lexical priming

${ }^{37}$ Associative priming
}

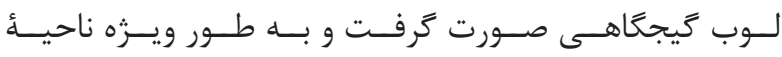

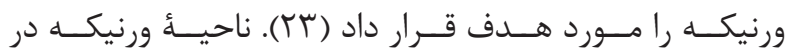

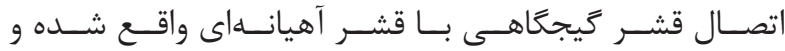

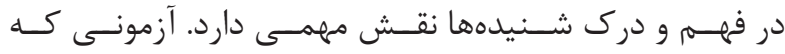

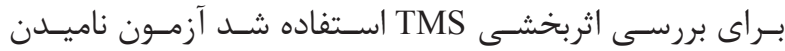

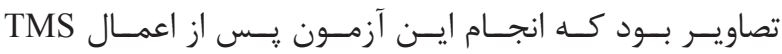

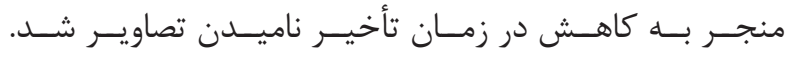

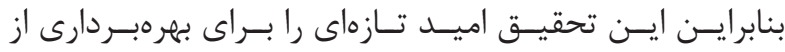

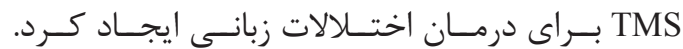
استفاده از TMS براى تداخل با قابليتهاى شناختى مربوط بله مخجه نر

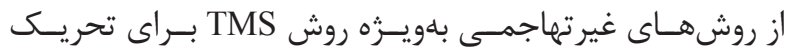

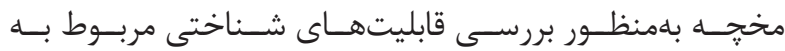

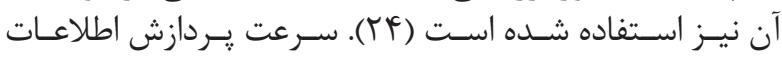

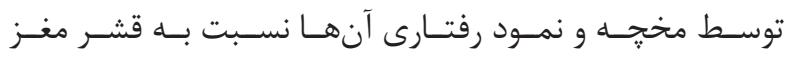

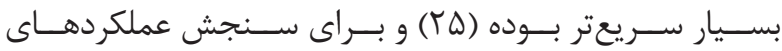

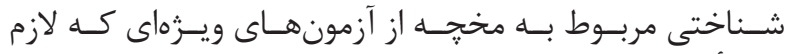

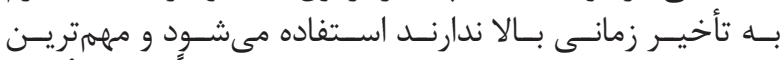

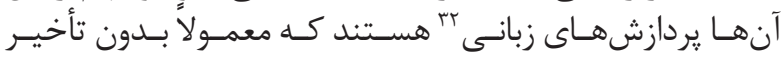

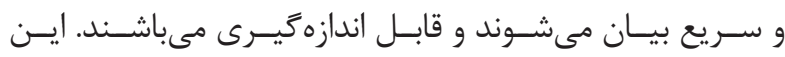

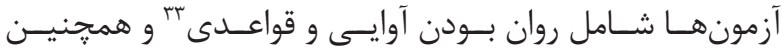

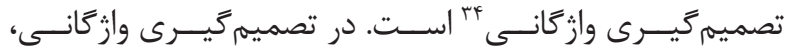

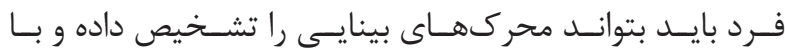

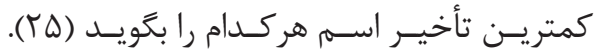

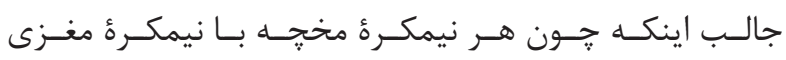

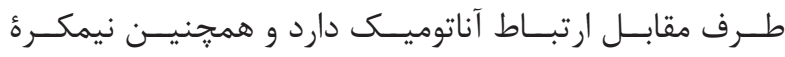

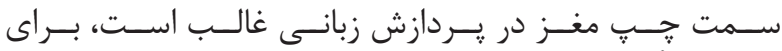

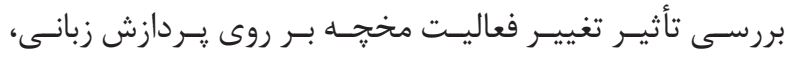

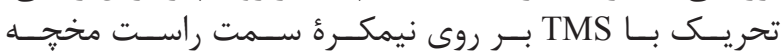

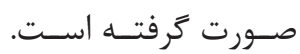

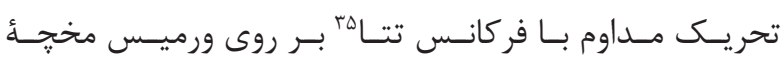

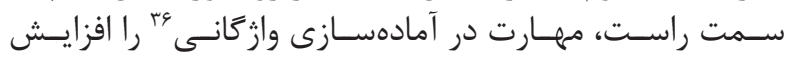

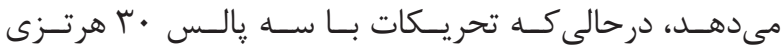

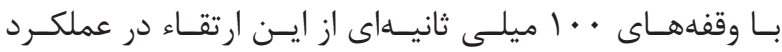

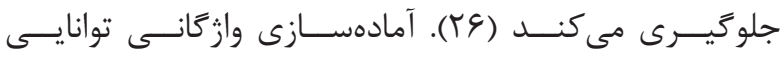

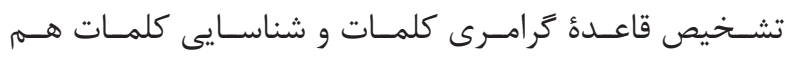

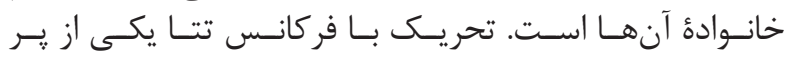

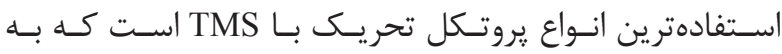

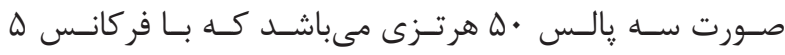

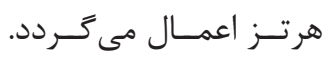
لازم بــه ذكـر اسـت كـهـ تحريـك مـداوم بــا فر كانسـ تتــا اثرات

\footnotetext{
${ }^{38}$ Emotion

${ }^{39}$ Mood

${ }^{40}$ Alertness

${ }^{41}$ International affective picture set

${ }^{42}$ Procedural learning
} 
طــول مــدت بيمـارى رابطــــ مسـتقيم دارد (Y) (F).

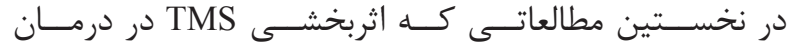

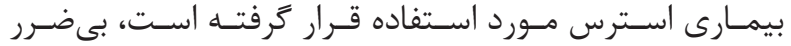

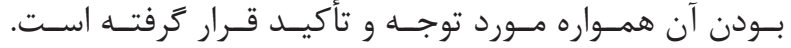

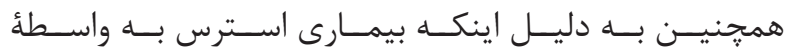

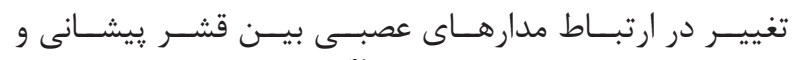

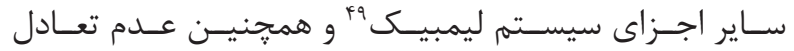

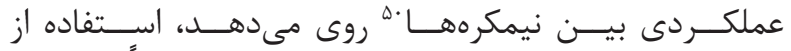

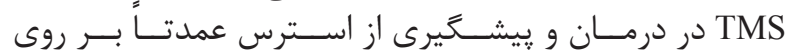

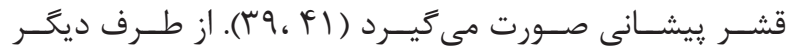

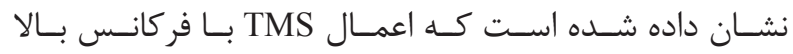

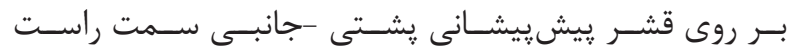

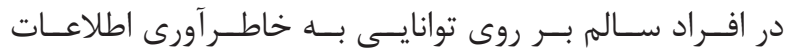

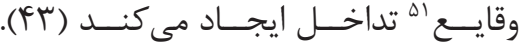

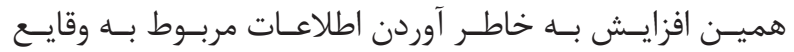

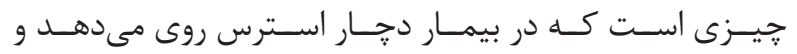

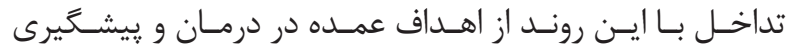

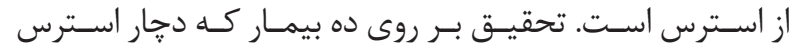

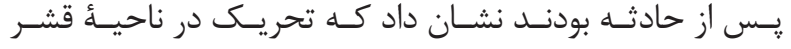

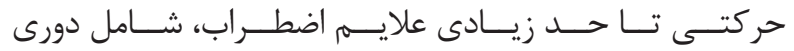

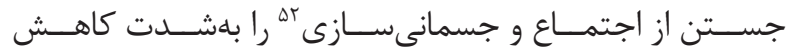

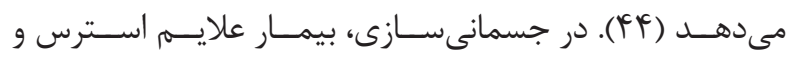

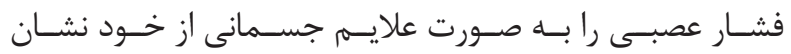

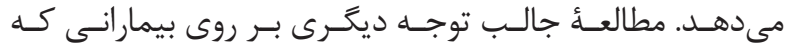

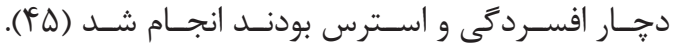

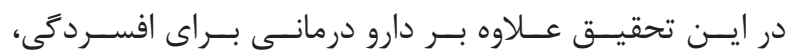

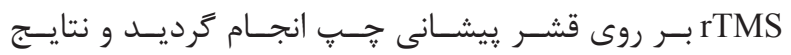

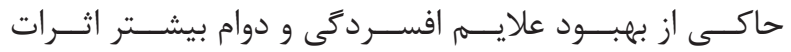

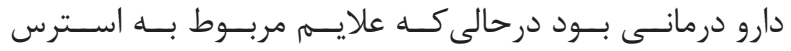

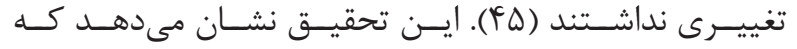

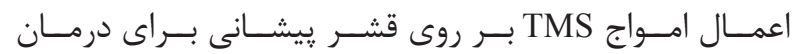

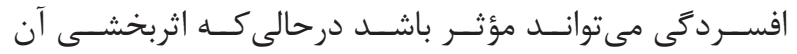

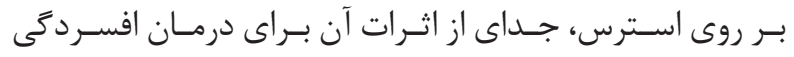

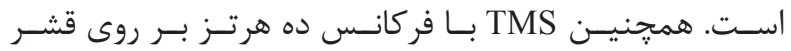

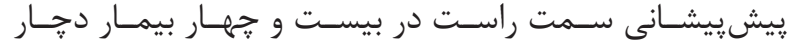

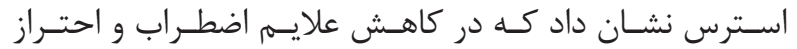

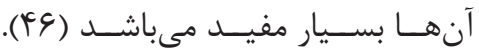

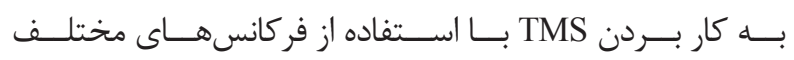

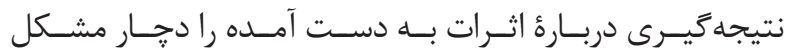

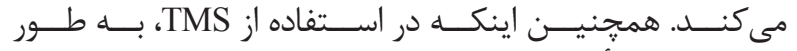

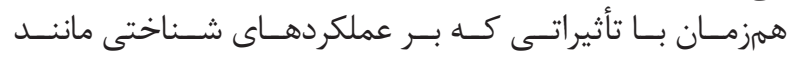

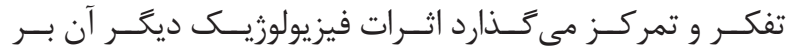

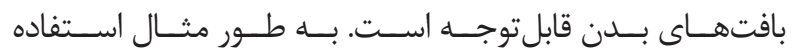

\footnotetext{
${ }^{43}$ Reaction time

${ }^{44}$ Single pulse rTMS

${ }^{45}$ Time perception

${ }^{46}$ Post-traumatic stress disorder

${ }^{47}$ Drug-naive PTSD patients
}

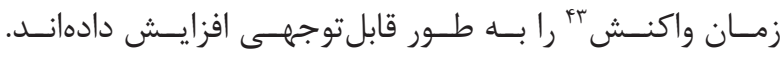

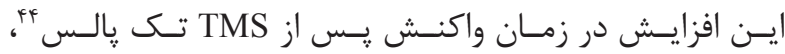

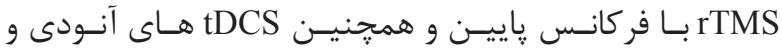

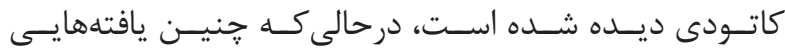

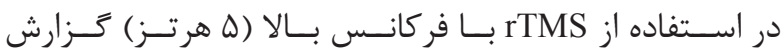
نشـده اسـت استه

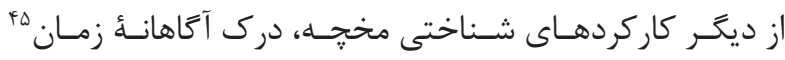

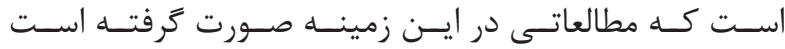


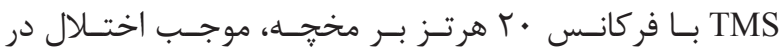

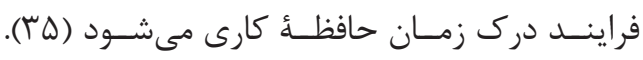

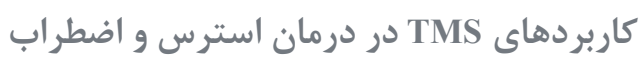

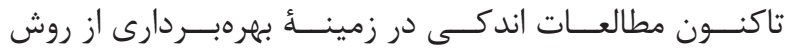

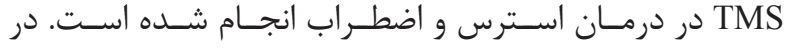

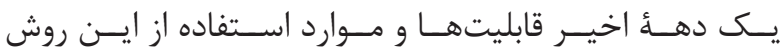

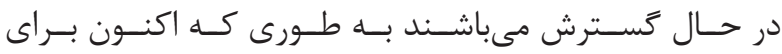

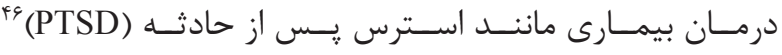

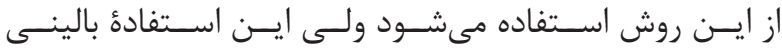

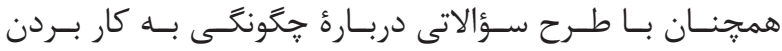

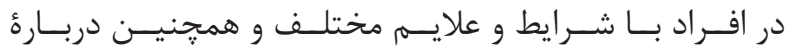

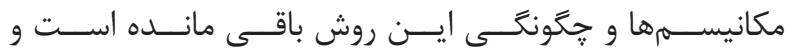

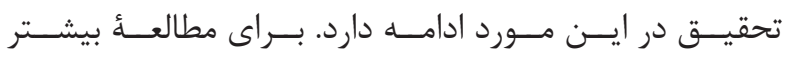

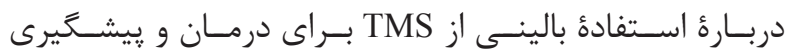

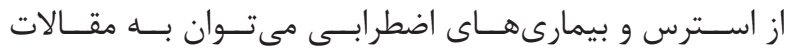

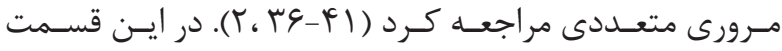

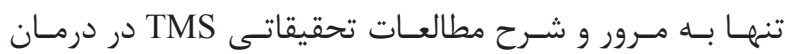

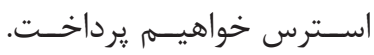

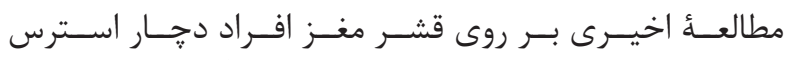

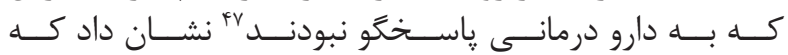

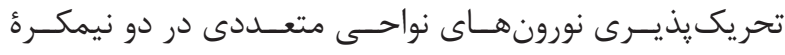

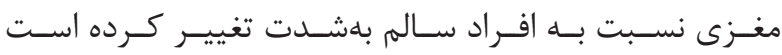

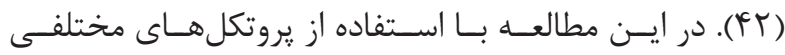

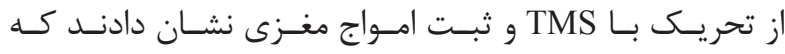

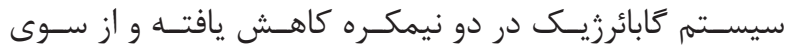

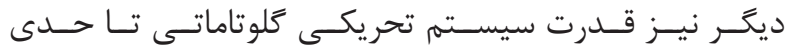

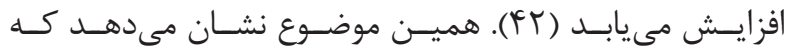

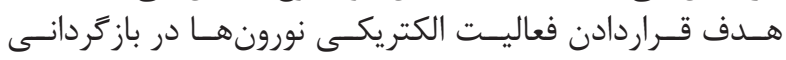

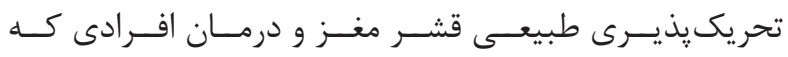

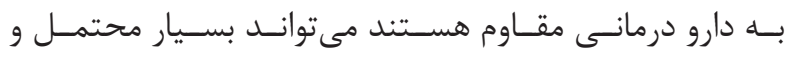

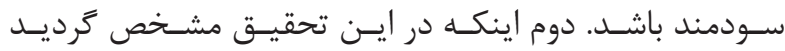

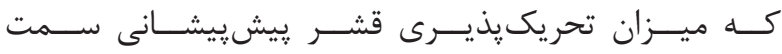

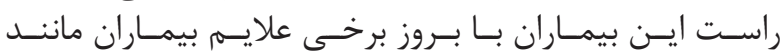

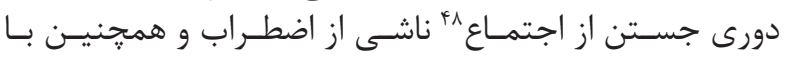

${ }^{48}$ Avoidance

${ }^{49}$ limbic system

${ }^{50}$ Interhemispheric imbalance

${ }^{51}$ Episodic memory retrieval

52 Somatization 


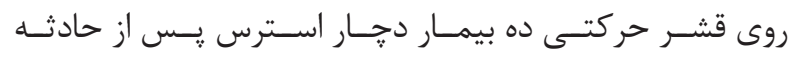

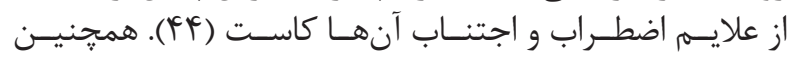

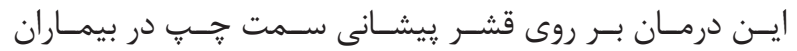

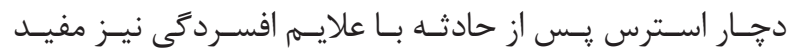

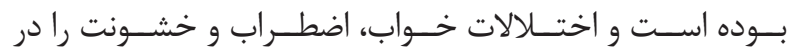

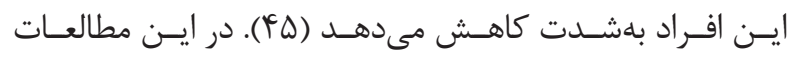

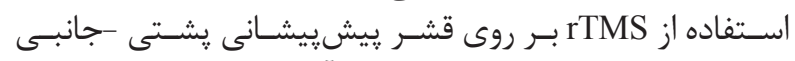

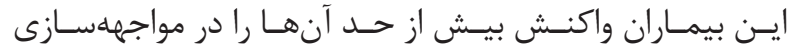

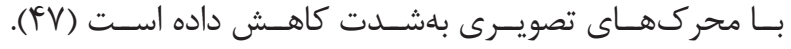

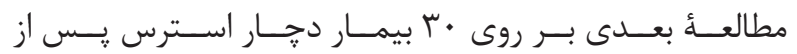

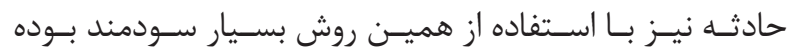

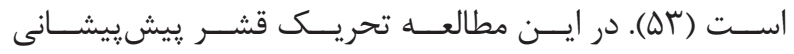

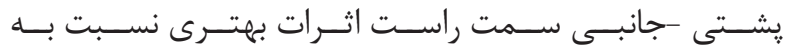
تحريـك هميـن قشـر در ســـت خــ

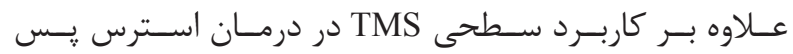

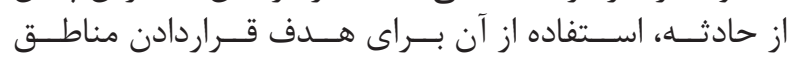

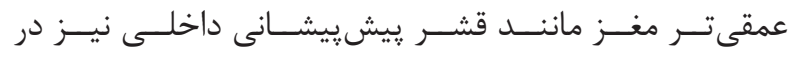

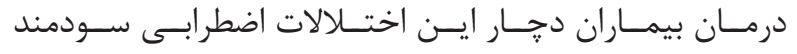

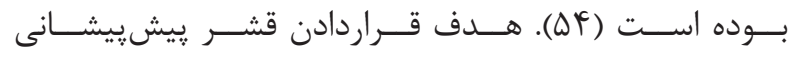

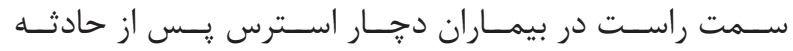

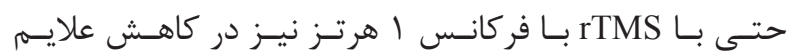

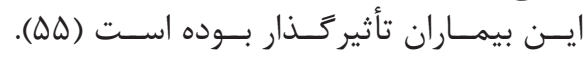

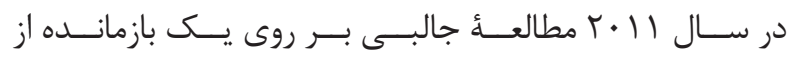

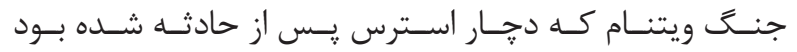

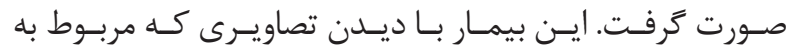

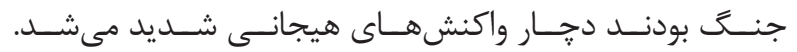

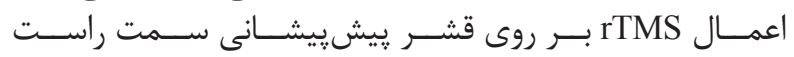

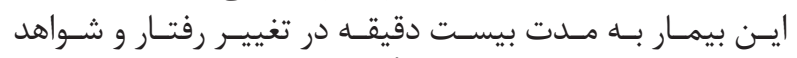

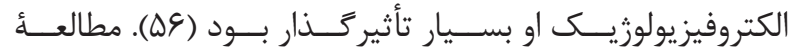

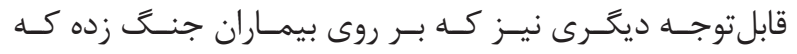

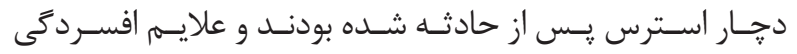

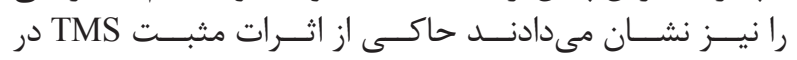

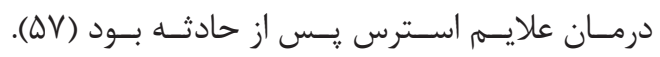

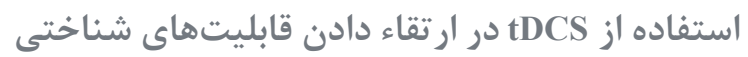

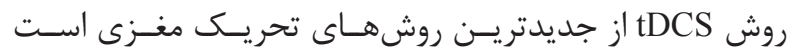

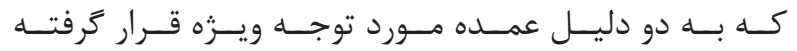

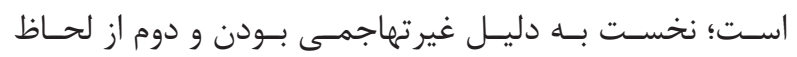

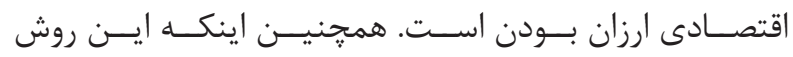

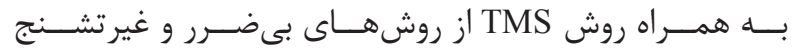

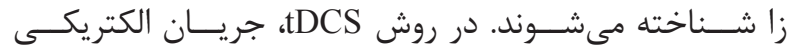

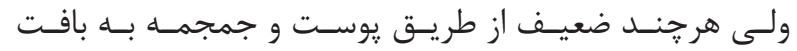

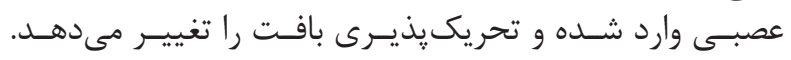

جََونَى عملكرد روش tDCS

يروتكل هــاى معمـول اسـتفاده از tDCS از طريـق دو الكتـــوود

${ }^{53}$ Hyperarousal symptoms

${ }^{54}$ Ventromedial prefrontal cortex

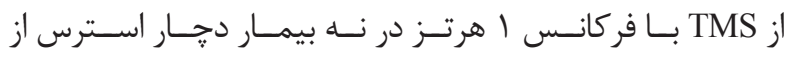

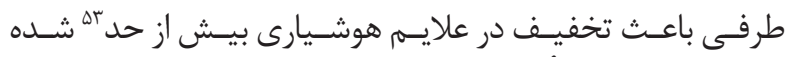

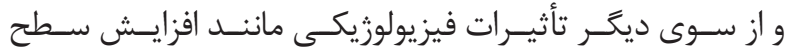

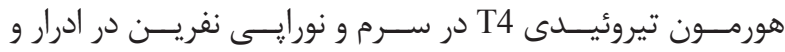

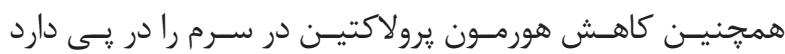
(FV)

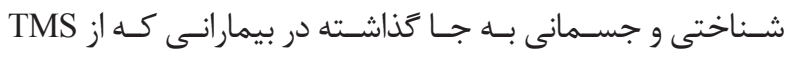

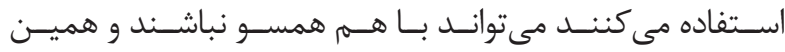

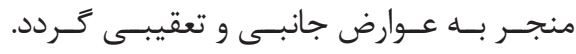

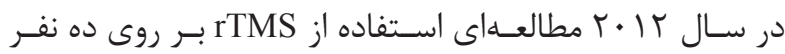

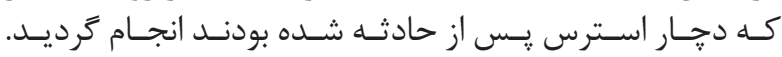

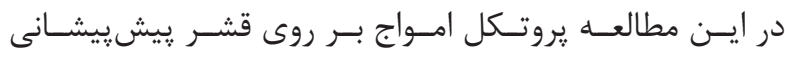

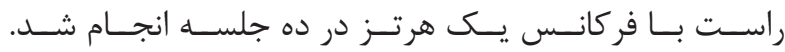

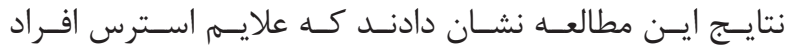

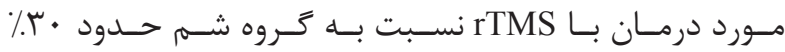

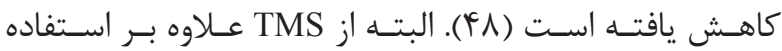

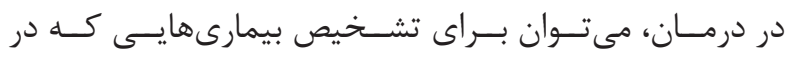

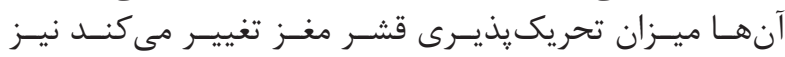

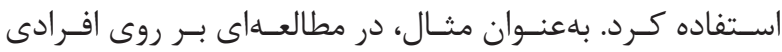

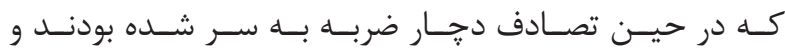

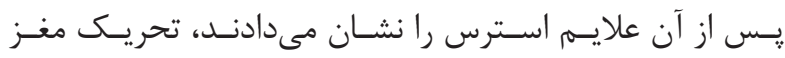

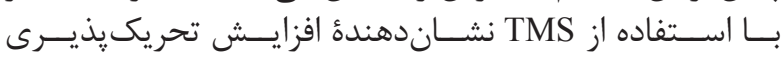

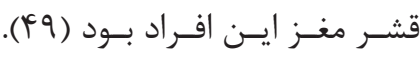
استفاده از TMS در درمان اختلال استرس يس إز حادثر

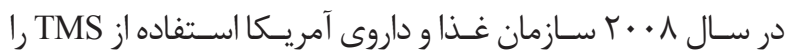

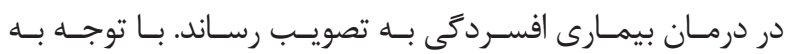

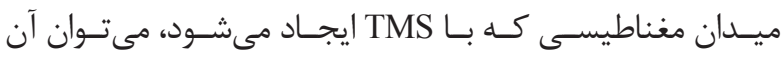

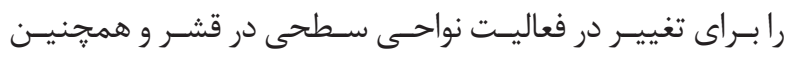

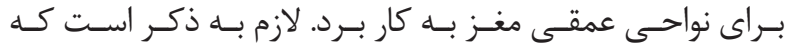

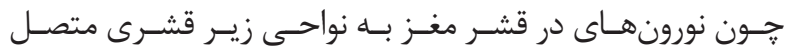

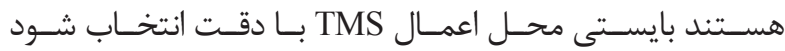

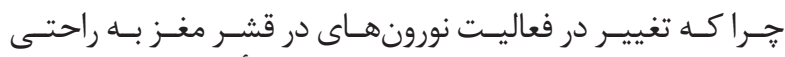

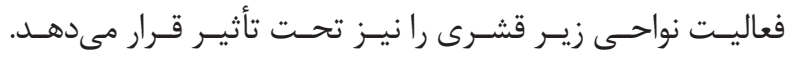

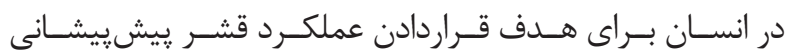

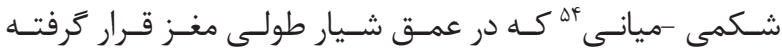

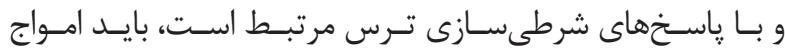

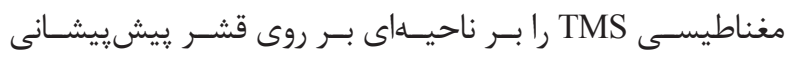

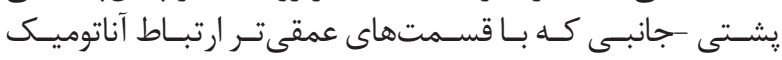

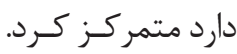

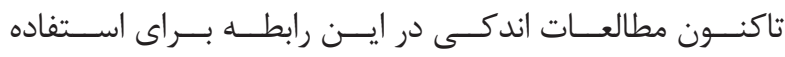

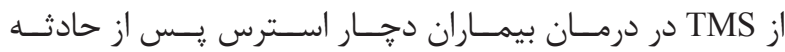

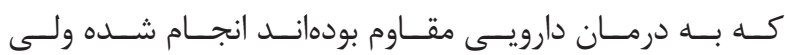

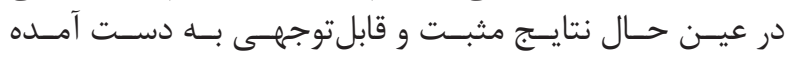

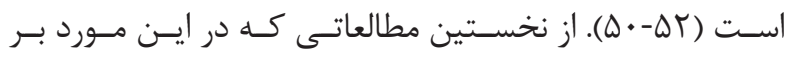

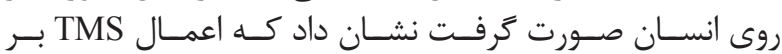




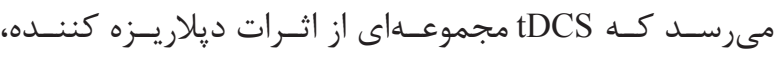

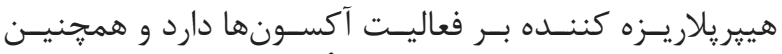

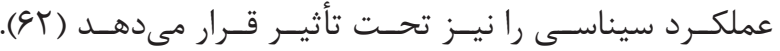
استفاده از tDCS بر روى قشر ييش ييشانى هشتى -جانبى

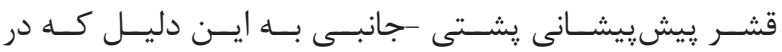

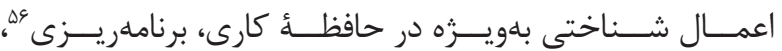

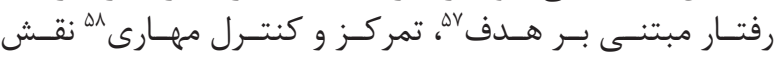

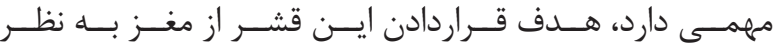

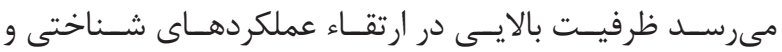

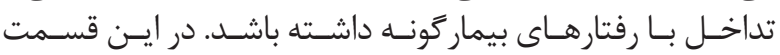

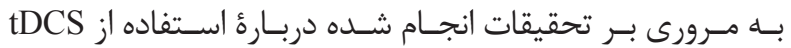

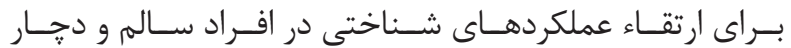

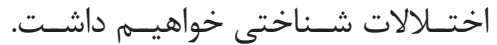

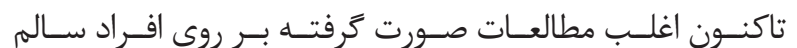

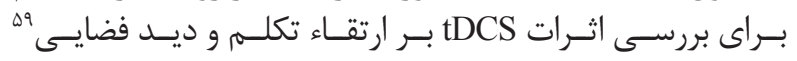

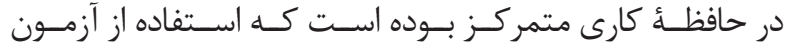

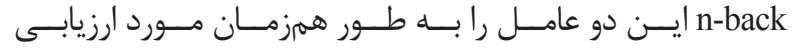

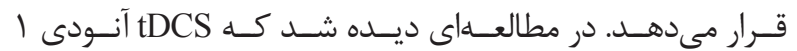

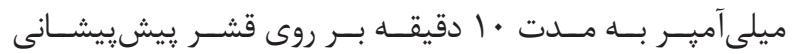

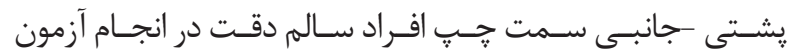

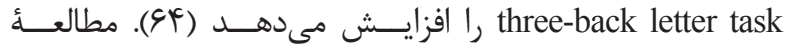

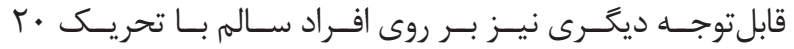

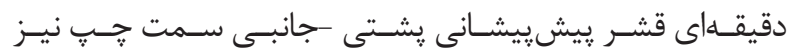

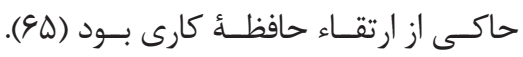

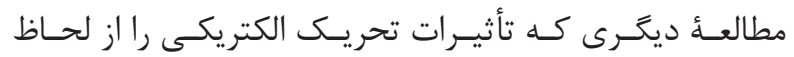

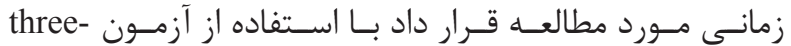

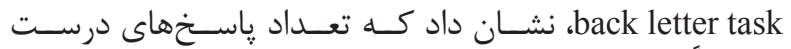

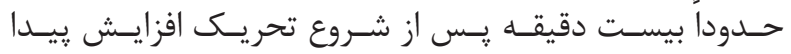

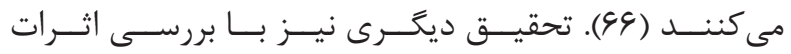

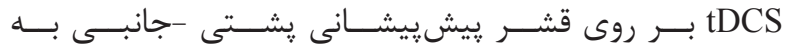

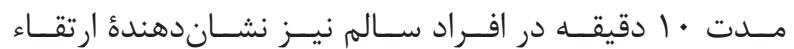

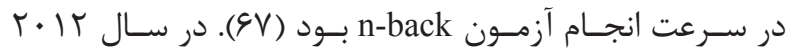

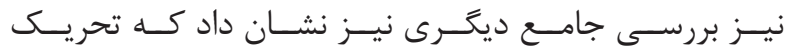

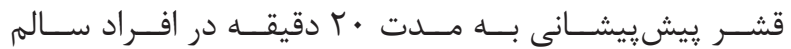

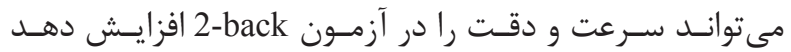

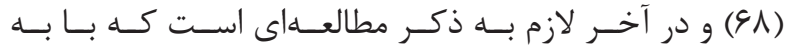

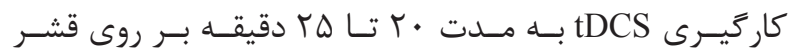

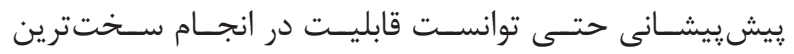

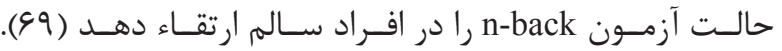

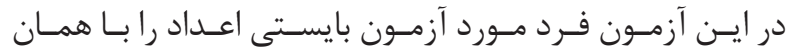

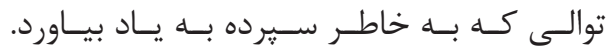

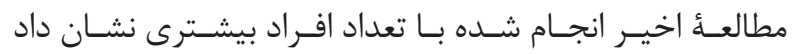

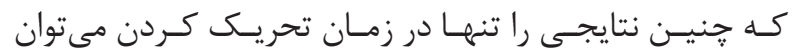

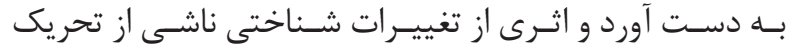

\footnotetext{
${ }_{55}^{55}$ Intracortical facilitation

${ }^{56}$ Planning
}

${ }^{57}$ Goal-directed behavior

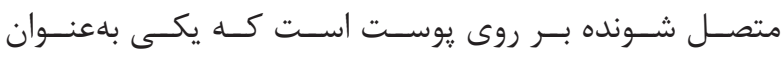

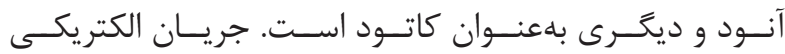

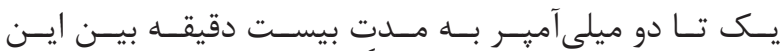

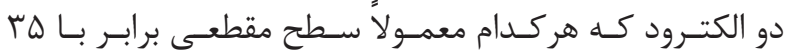

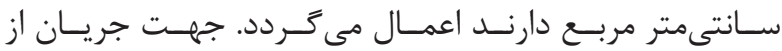

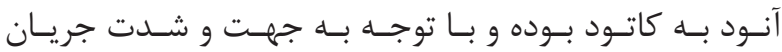

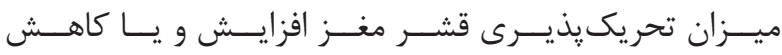

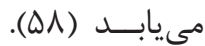

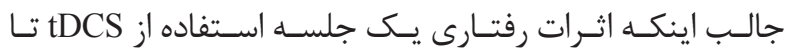

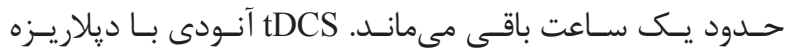

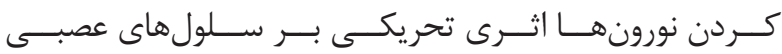

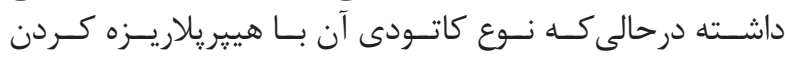

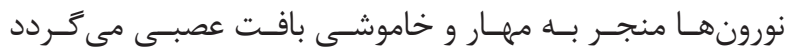

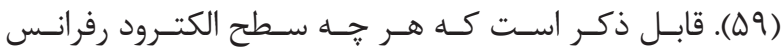

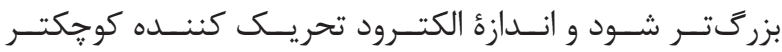

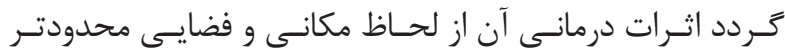

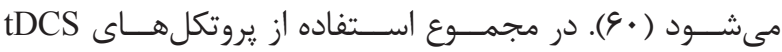

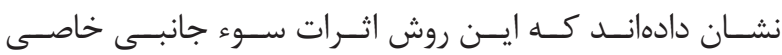

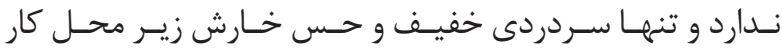

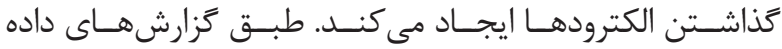

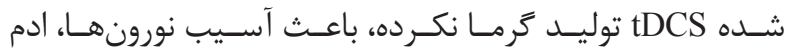

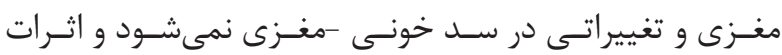

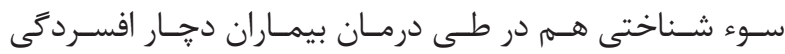

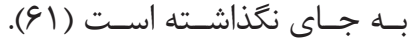

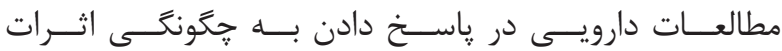

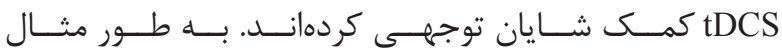

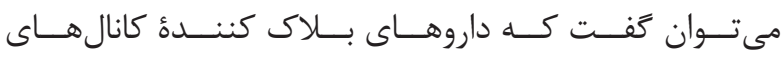

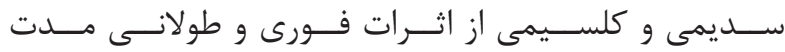

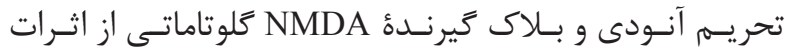

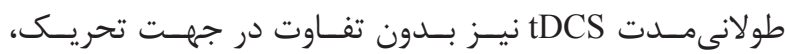

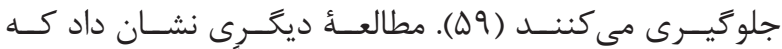

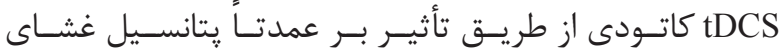

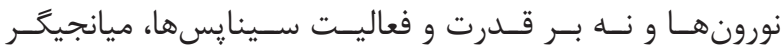

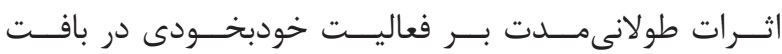

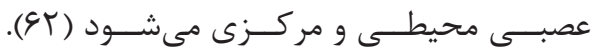

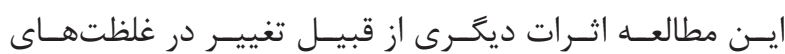

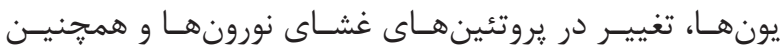

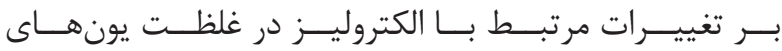

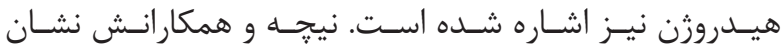

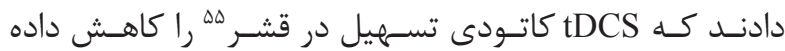

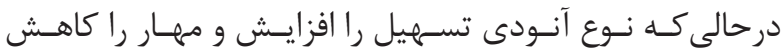

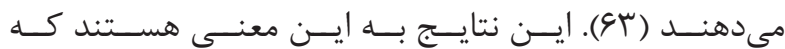

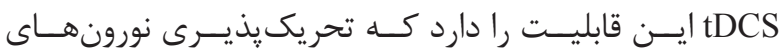

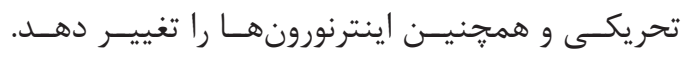

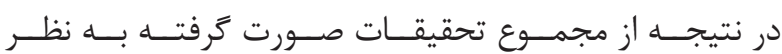

\footnotetext{
${ }^{58}$ Inhibitory control

${ }^{59}$ Visuospatial
} 


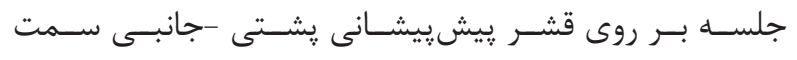

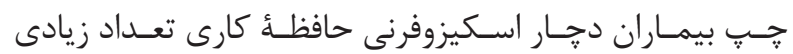

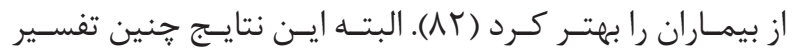

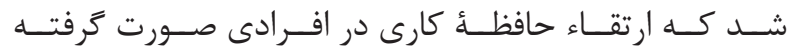

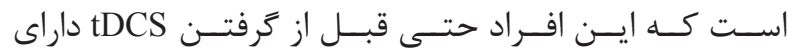

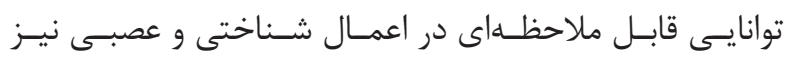

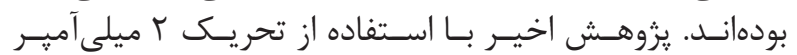

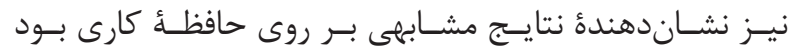

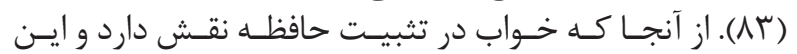

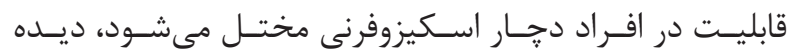

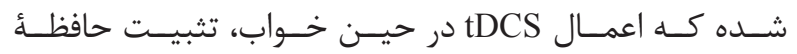

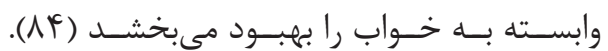

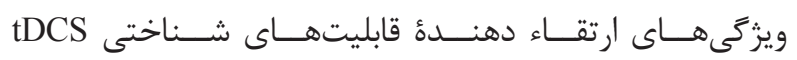

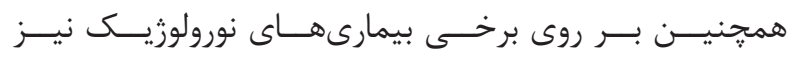

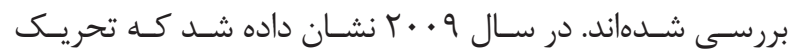

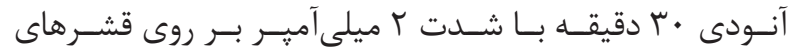

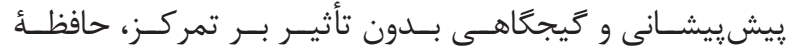

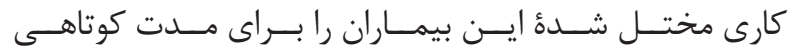

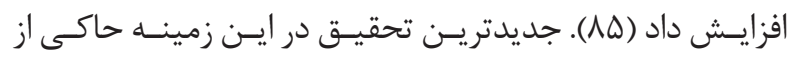

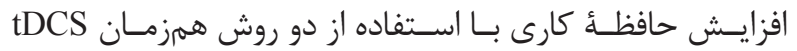

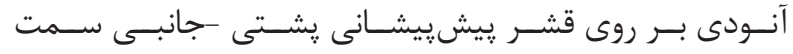

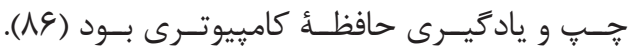

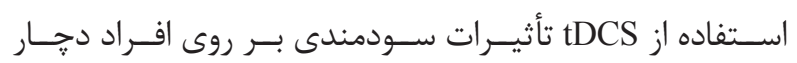

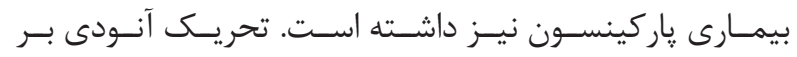

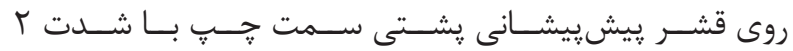

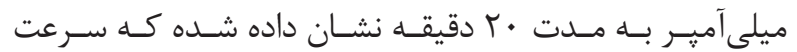

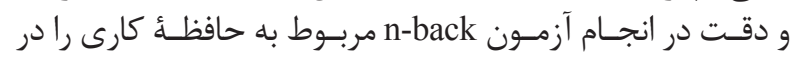

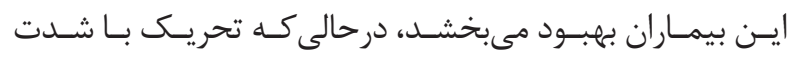

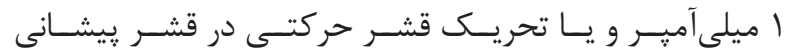

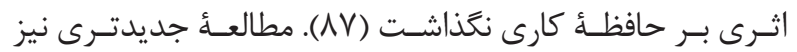

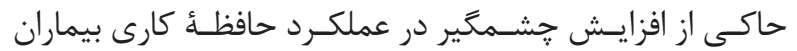

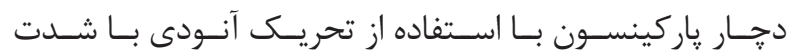

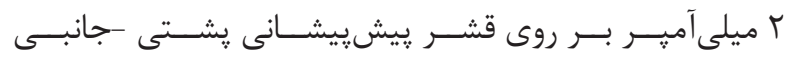

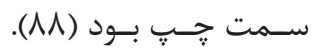

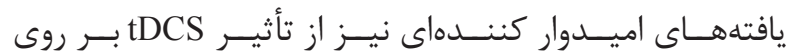

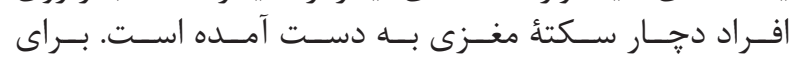

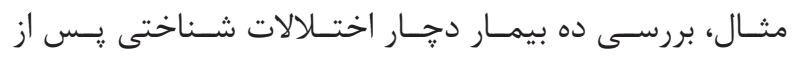

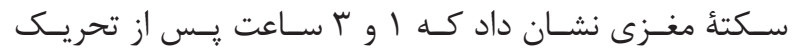

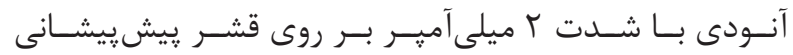

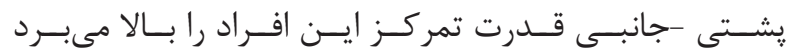

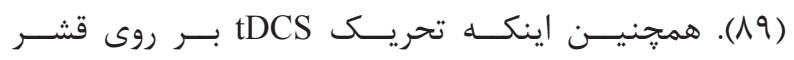

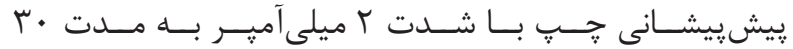

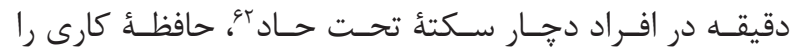

بــه طـور :جشــمخيرى افزايـش در داد ( • (9).

\footnotetext{
${ }^{60}$ Implicit learning

${ }^{61}$ Procedural learning

${ }^{62}$ Subacute stroke
}

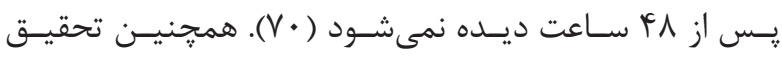

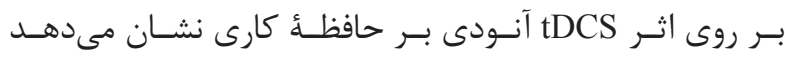

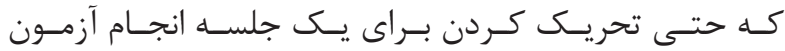
digit span forward

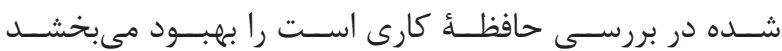

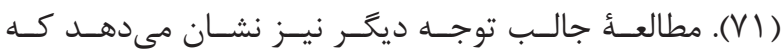

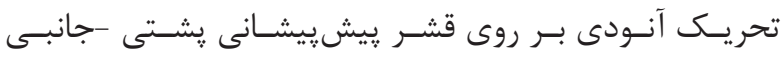

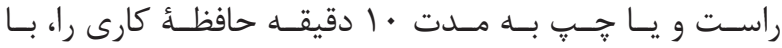

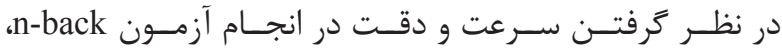

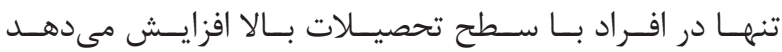

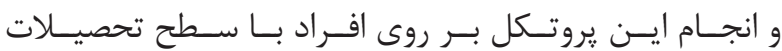

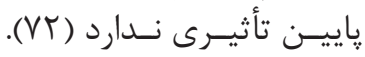

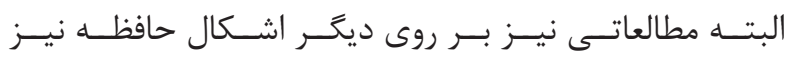

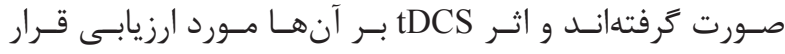

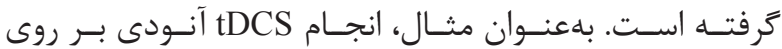

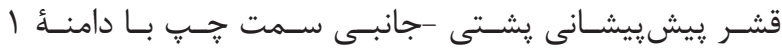

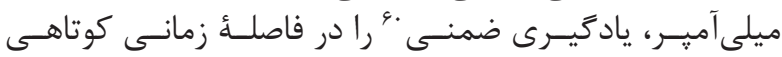

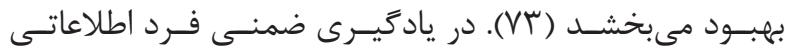

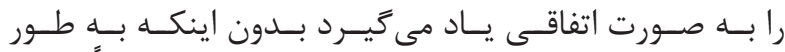

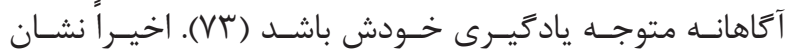

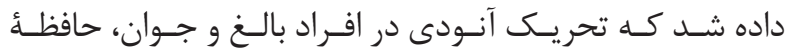

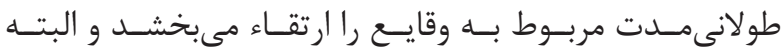

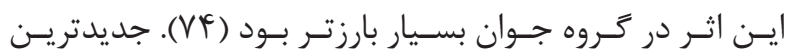

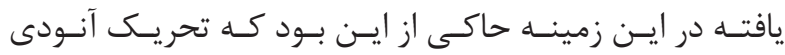

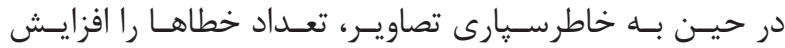

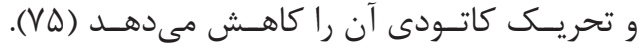

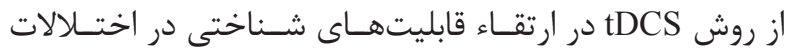

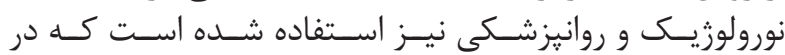

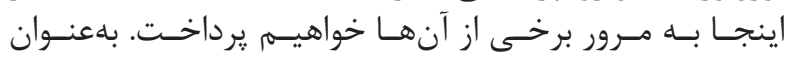

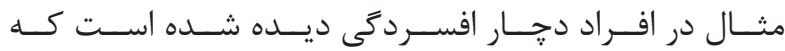

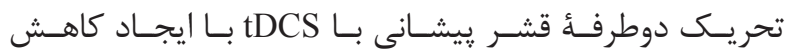

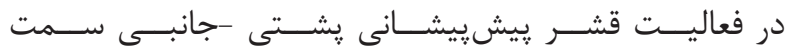

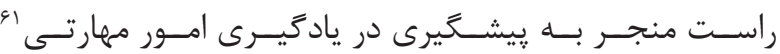

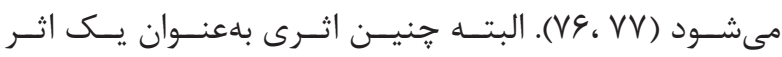

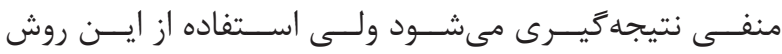

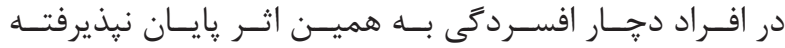

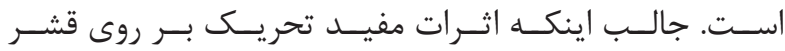

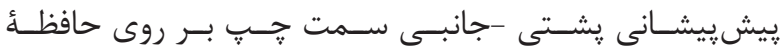

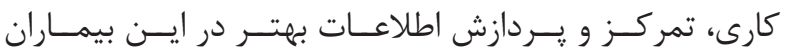

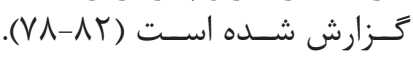

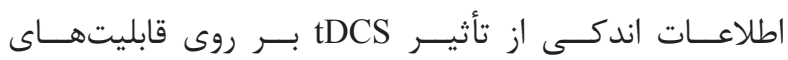

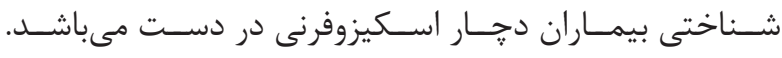

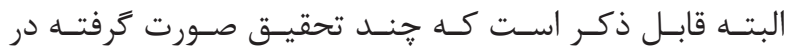

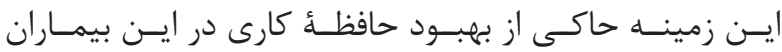

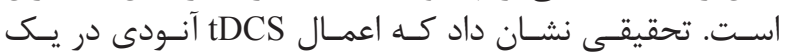


استفاده از tcDCS براى ارتقاء حافظة كارى وابسته به

مخجهـ

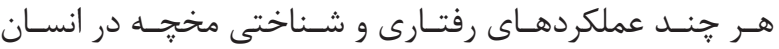

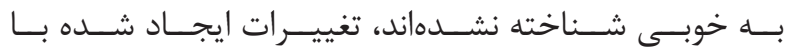

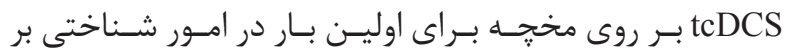

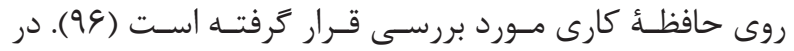

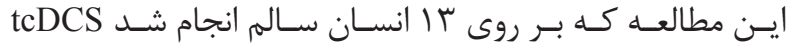

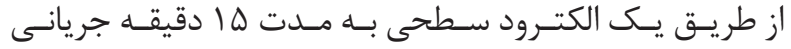

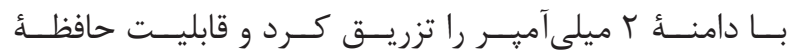

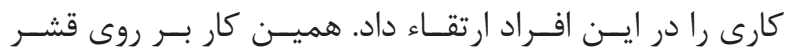

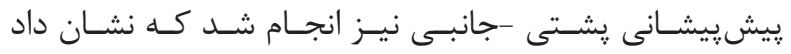

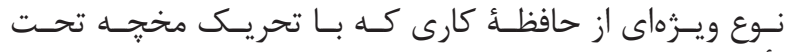

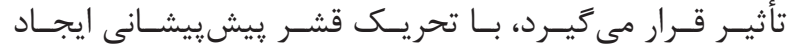

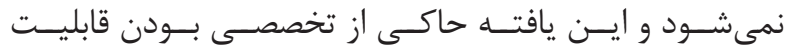

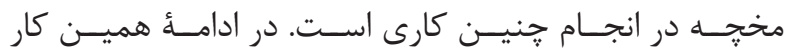

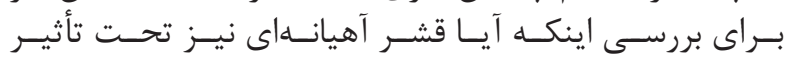

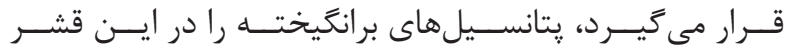

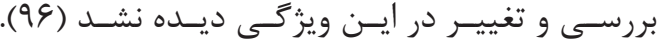

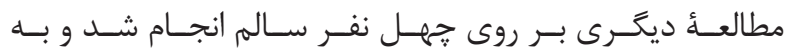

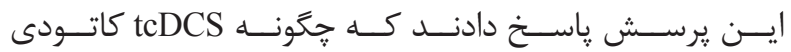

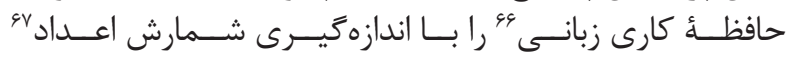

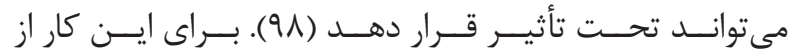

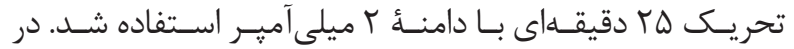

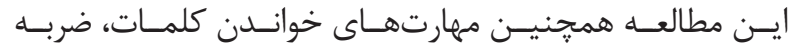

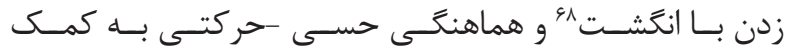

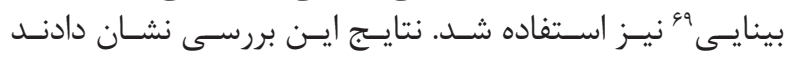

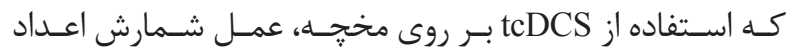

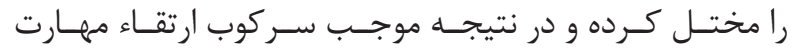

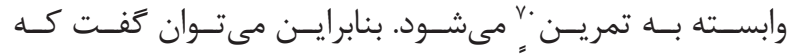

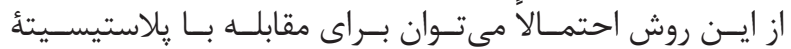

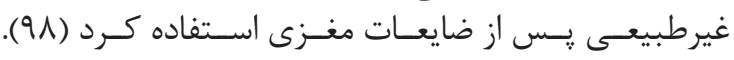

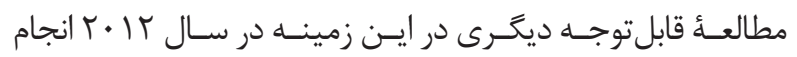

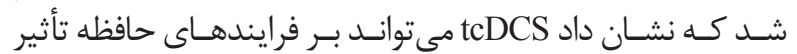

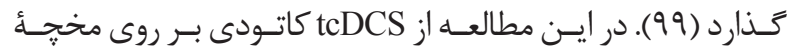

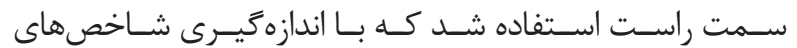

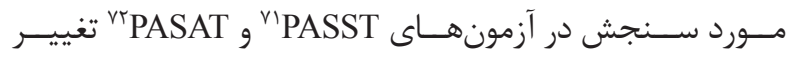

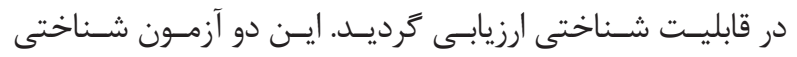

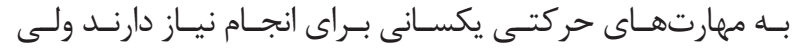

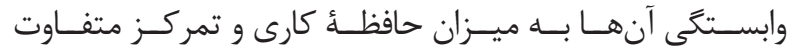

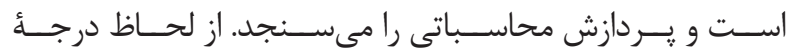

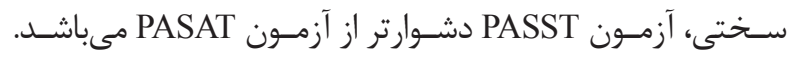

${ }^{63}$ Visual recognition memory task

${ }^{64}$ Mood control

${ }^{65}$ Transcranial cerebellar direct current stimulation

${ }^{66}$ Verbal working memory

${ }^{67}$ Digit span

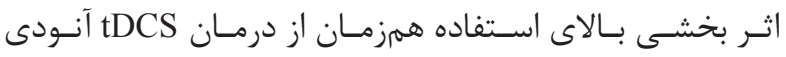

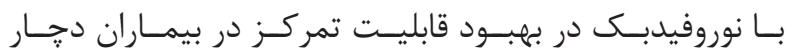

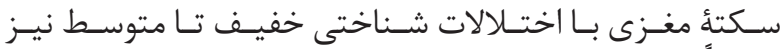

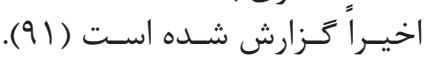
استفاده از tDCS بر روى قشر تيجكًاهى

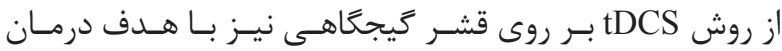

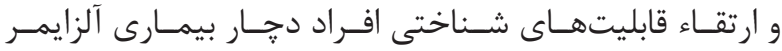

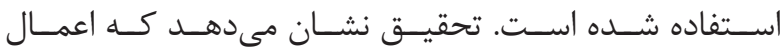

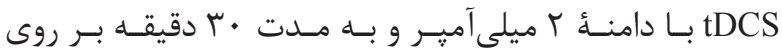

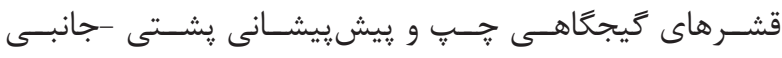

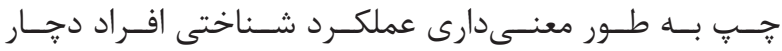

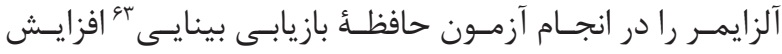

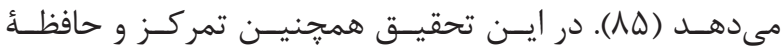

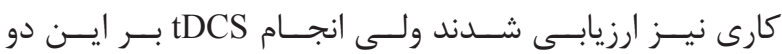

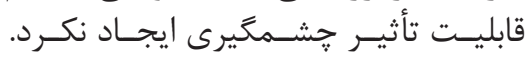
استفاده از tDCS بر روى مخجه

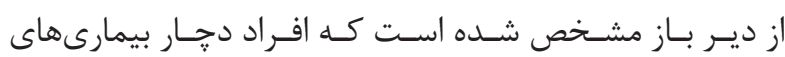

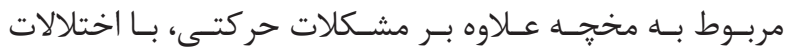

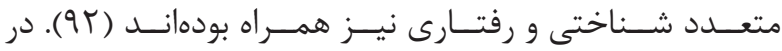

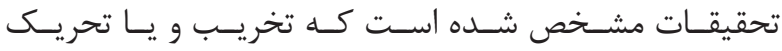

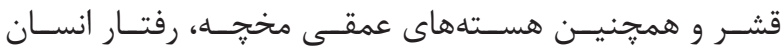

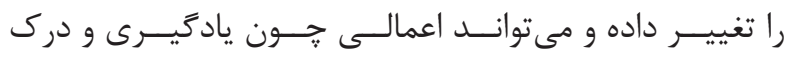
حـواس را دجــار اختـلال كنــــ (بهو).

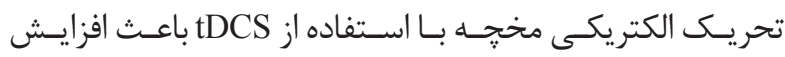

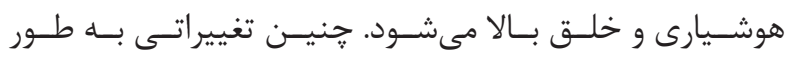

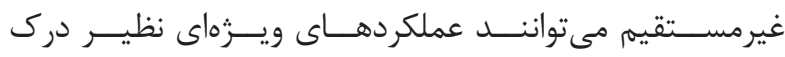

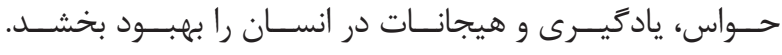

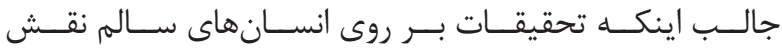

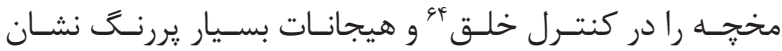

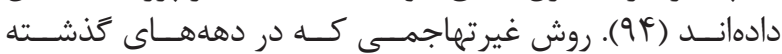

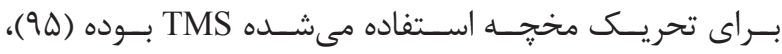

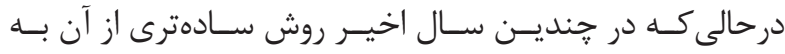

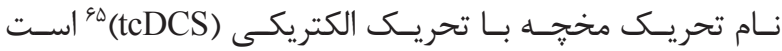

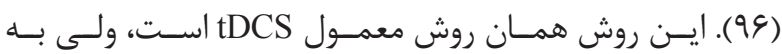

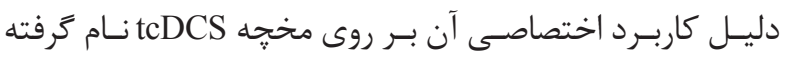

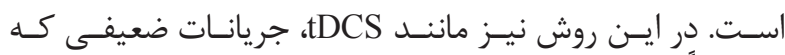

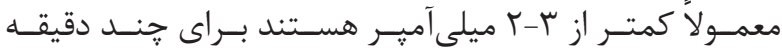

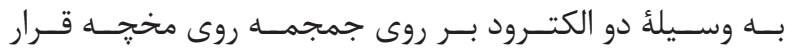

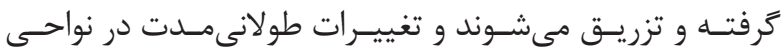

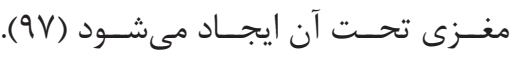

\footnotetext{
${ }^{68}$ Finger tapping

${ }^{69}$ Visually cued sensorimotor task

${ }^{70}$ Practice-dependent proficiency increase

${ }^{71}$ Paced Auditory Serial Subtraction Task

${ }^{72}$ Paced Auditory Serial Addition Task
} 


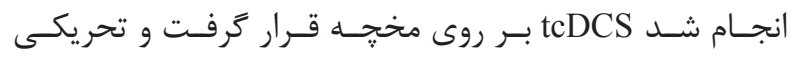

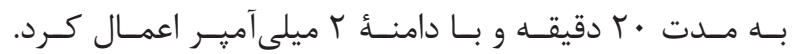

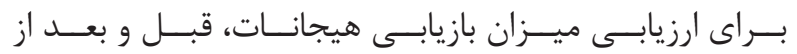
tcDCS

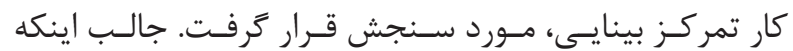

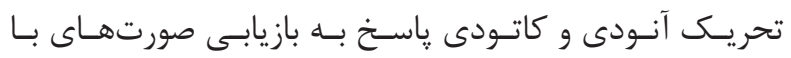

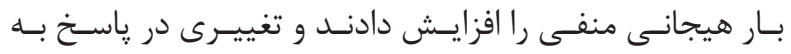

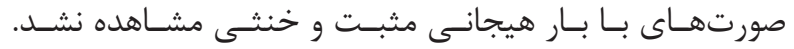

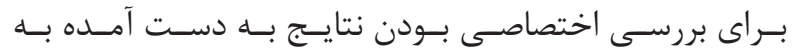

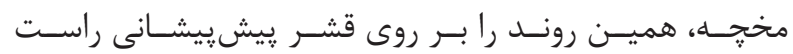

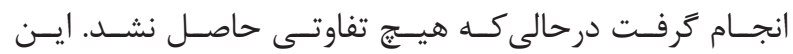

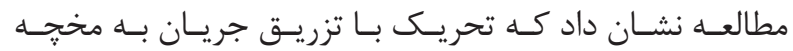

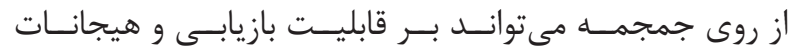

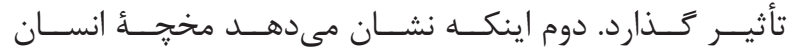

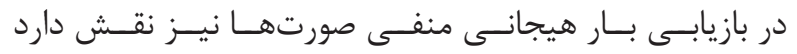

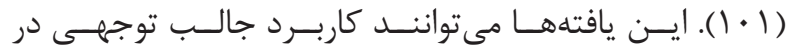

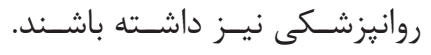

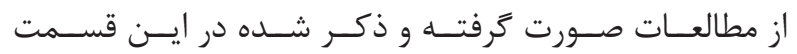

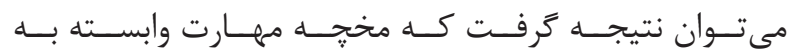

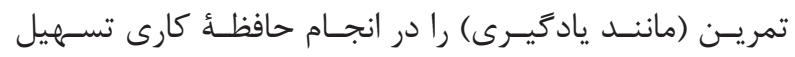

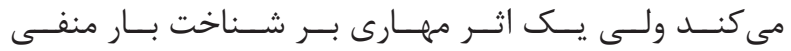

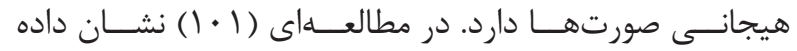

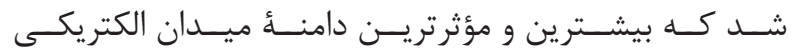

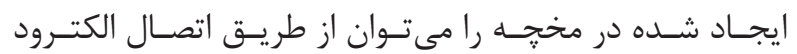

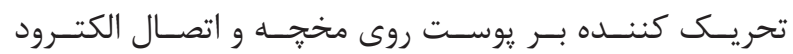

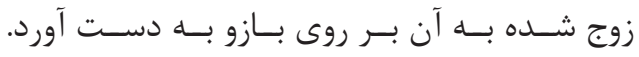

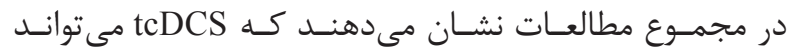

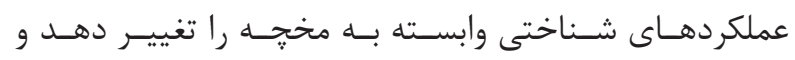

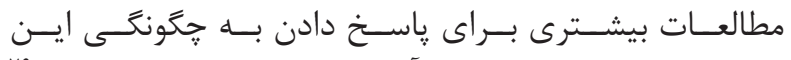

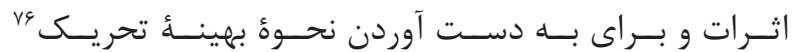

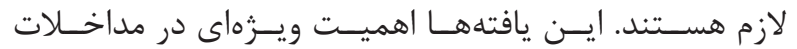

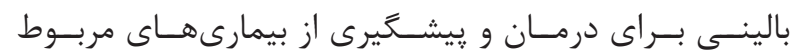
بــه مخجزهـه دارنـد.

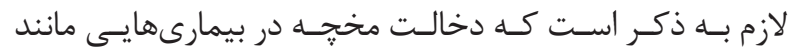

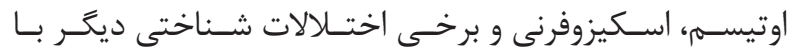

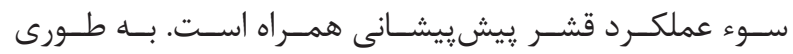

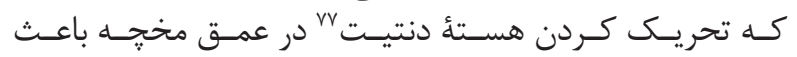

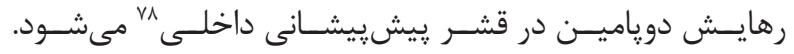

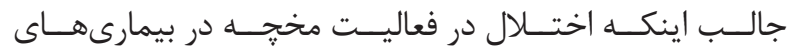

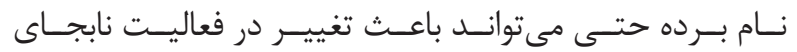

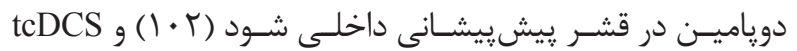

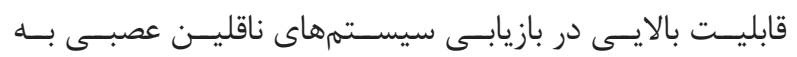

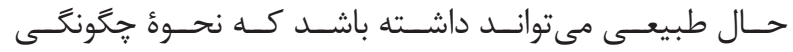

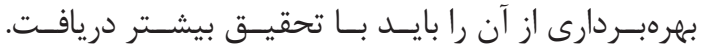

\footnotetext{
${ }^{73}$ Serial reaction time task

${ }^{74}$ Emotion recognition

${ }^{75}$ Facial emotion recognition
}

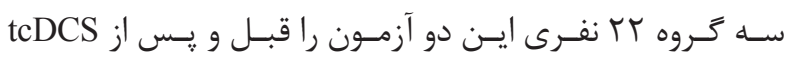

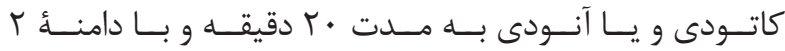

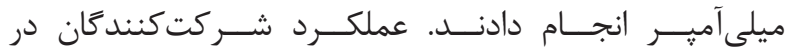

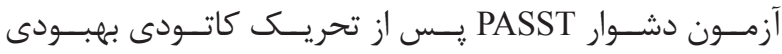

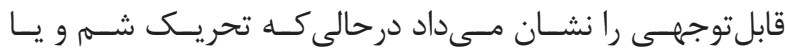

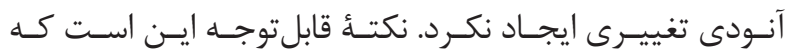

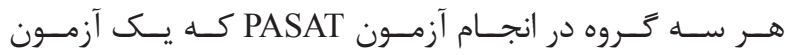

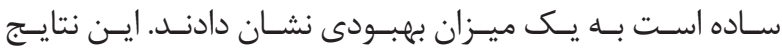

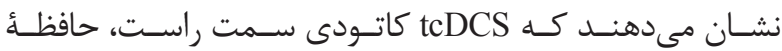

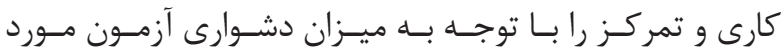

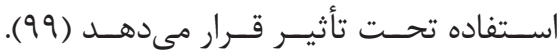

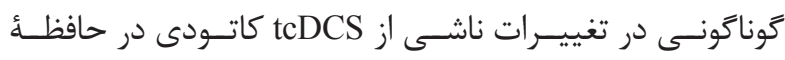

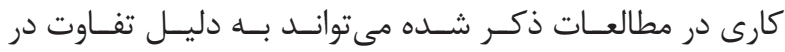

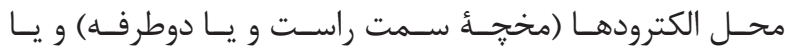

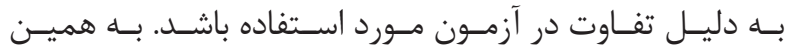

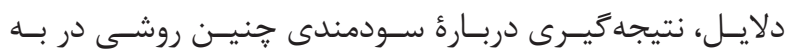

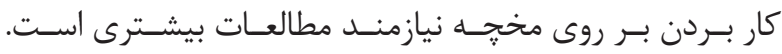
استفاده از tcDCS براى ارتقاء ياد تيرى وابسته به مخجه

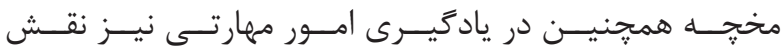

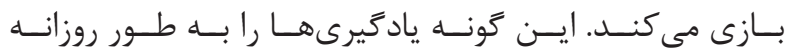

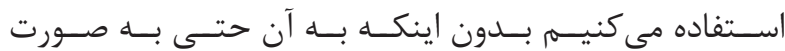

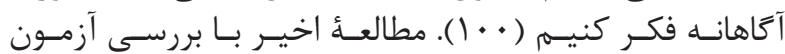

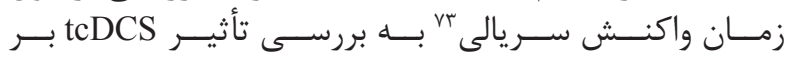

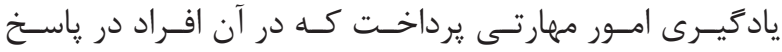

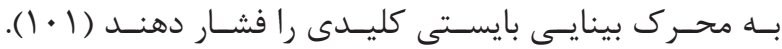

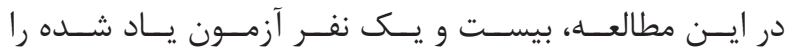

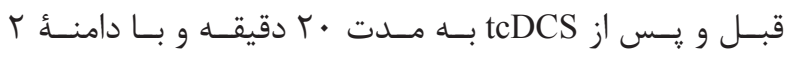

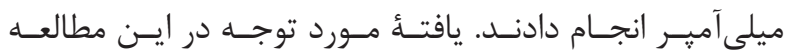

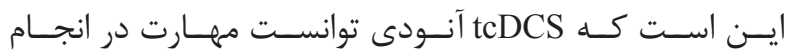

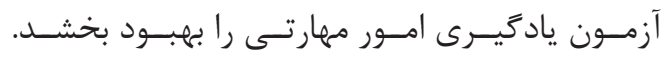

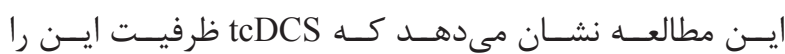

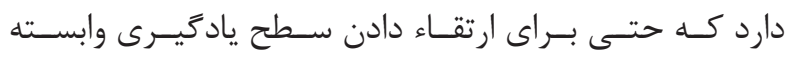

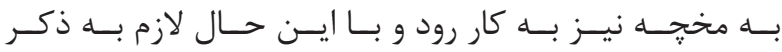

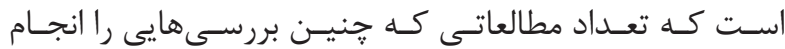

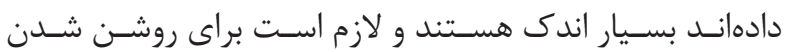

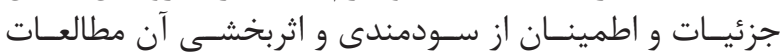
بيشـترى طراحسى و انجـام شــوند.

استفاده از tcDCS براى ارتقاء عملكرد بازيابى هيجانات

وابسته بله مخته

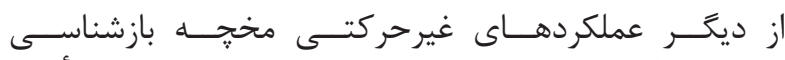

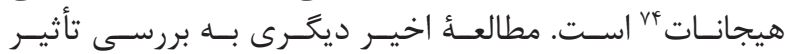

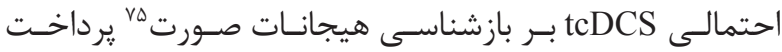

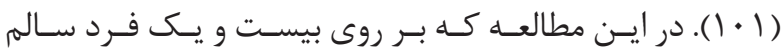

\footnotetext{
${ }^{76}$ Optimum stimulation settings

${ }^{77}$ Dentate nucleus

${ }^{78}$ Medial prefrontal cortex
} 


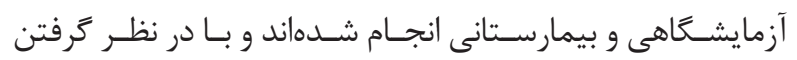

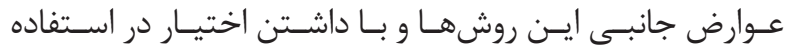

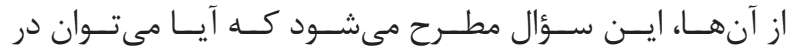

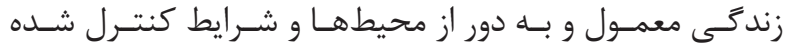

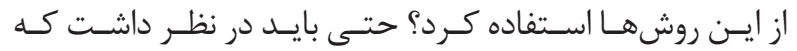

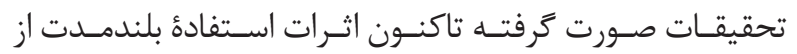

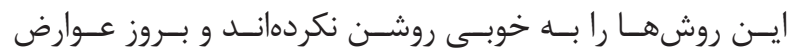

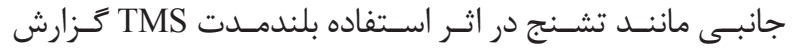

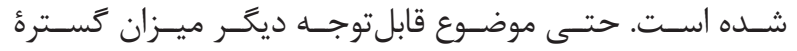

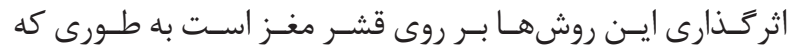

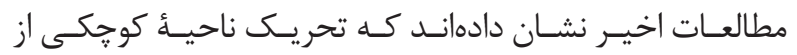

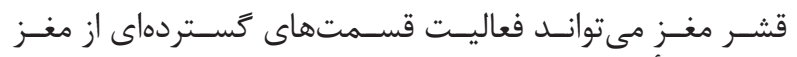

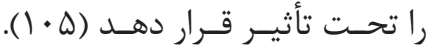

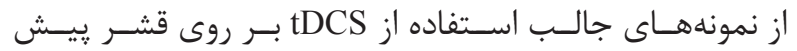

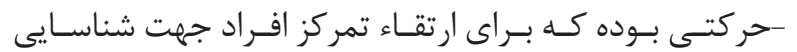

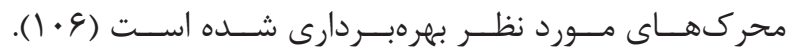

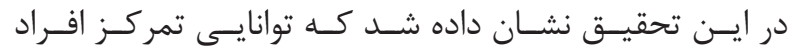

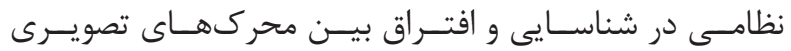

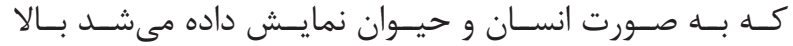

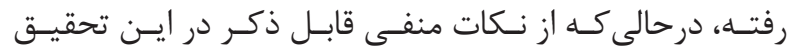

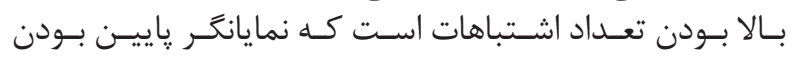

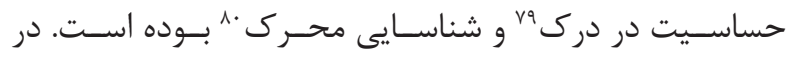

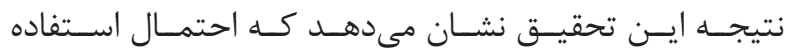

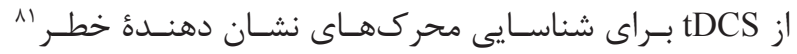

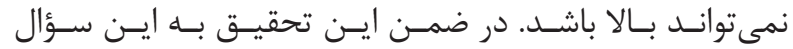

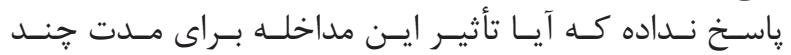

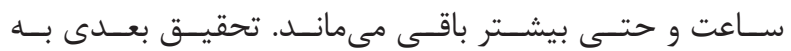

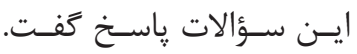

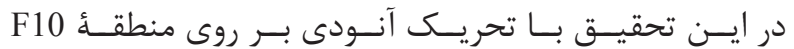

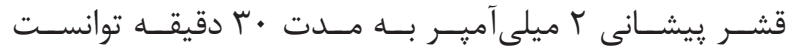

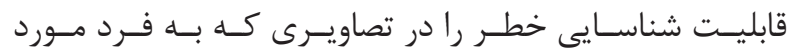

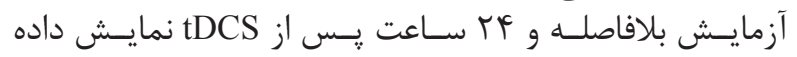

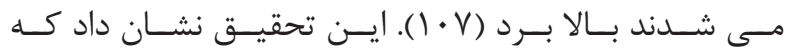

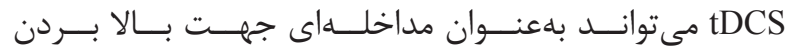

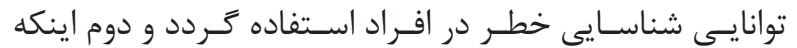

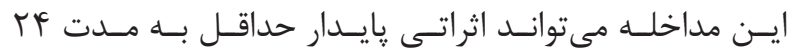

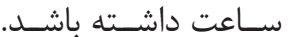

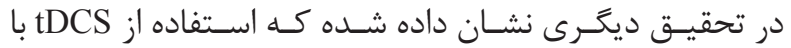

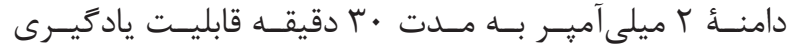

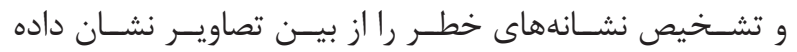

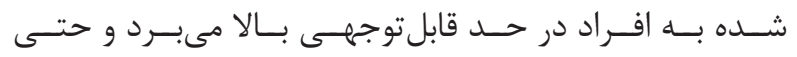

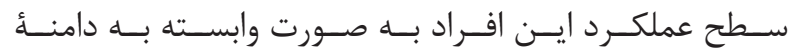
tDCS

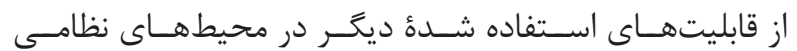
${ }^{79}$ Perceptual sensitivity

${ }^{80}$ Signal detection

${ }^{81}$ Threat detection

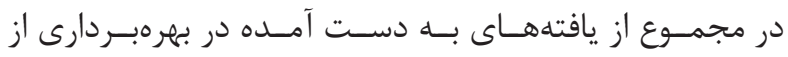

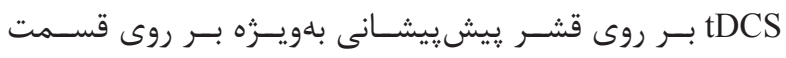

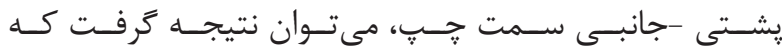

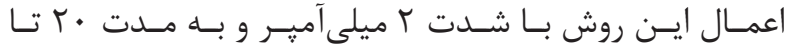

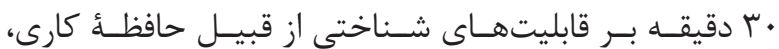

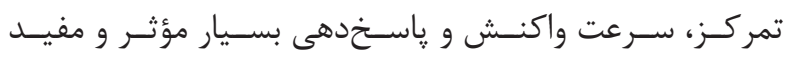

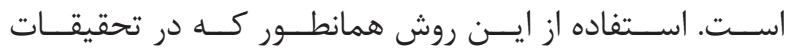

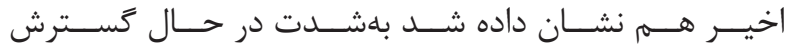

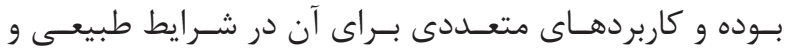

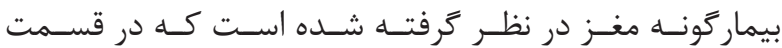

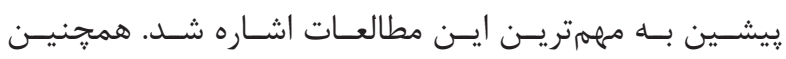

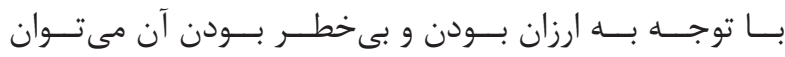

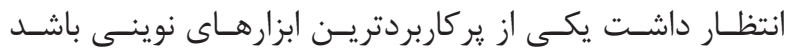

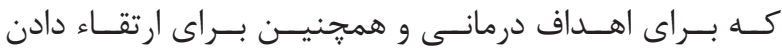

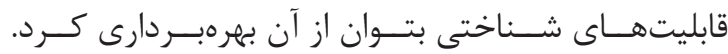
استفاده از روشهاى تحريك كننده غيرتهاجمى مغز براى ارتقاء قابليتهاى شناختى نيروهاى نظامى رونى

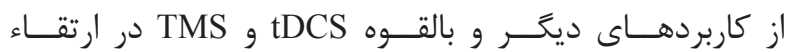

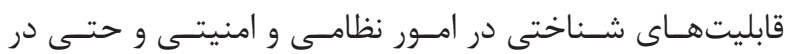

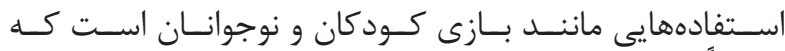

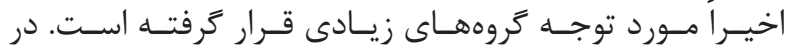

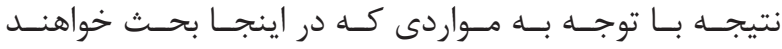

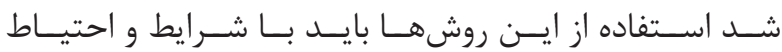

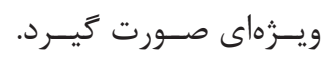

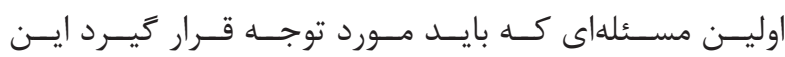

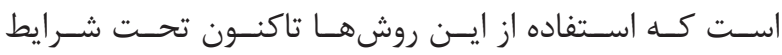

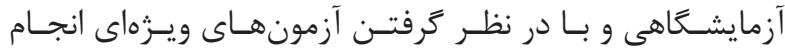

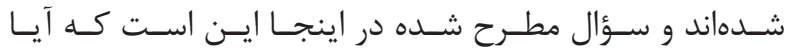

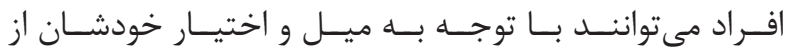

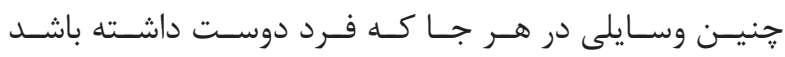

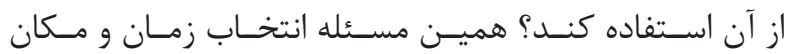

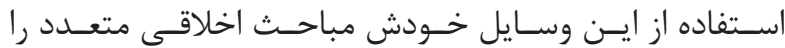

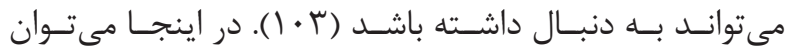

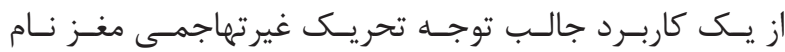

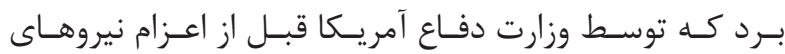

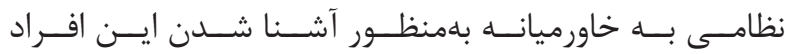

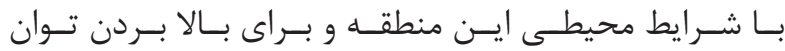

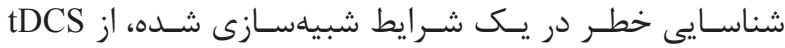

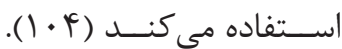

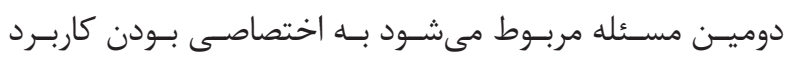

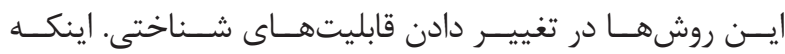

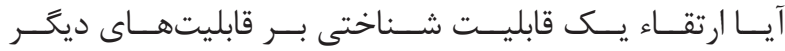

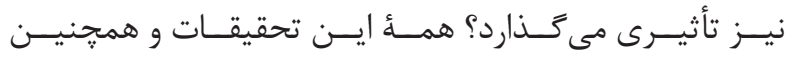

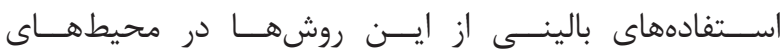




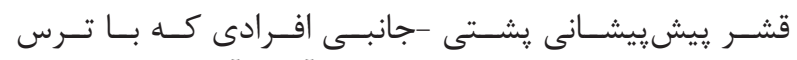

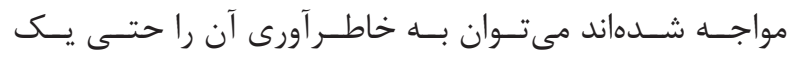

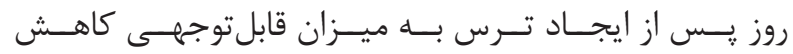

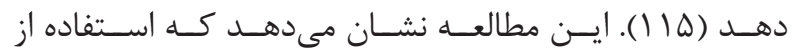

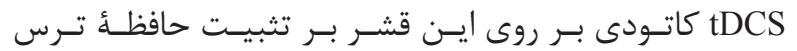

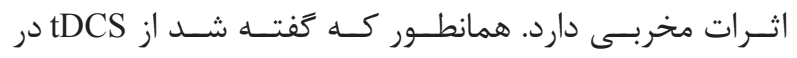

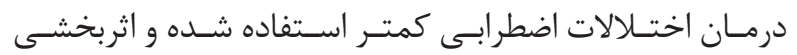

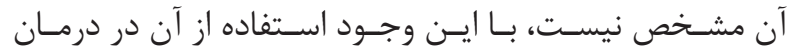

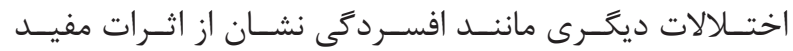

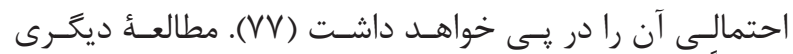

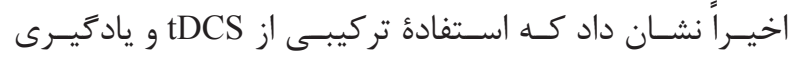

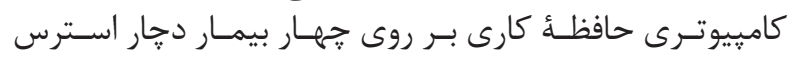

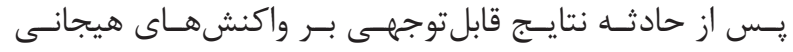

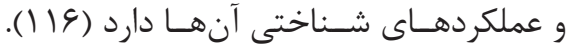

مقايسة استفاده از TMS و tDCS

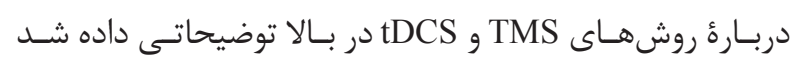

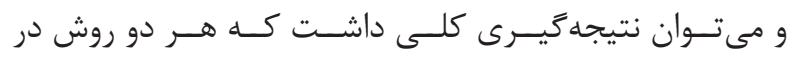

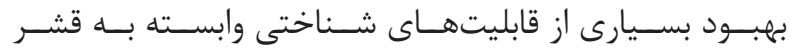

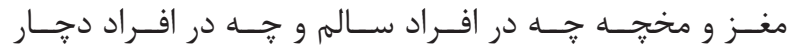

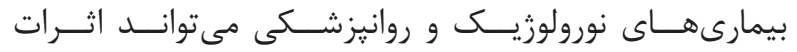

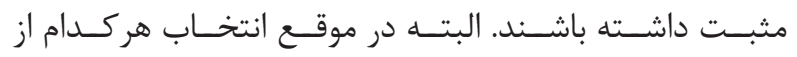

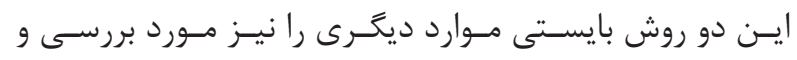

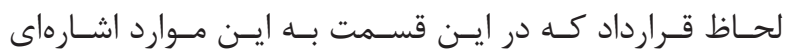
خواهـد شـد (IV) (IV)

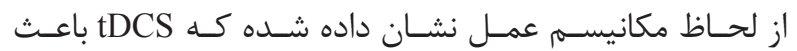

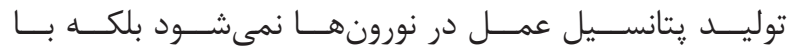

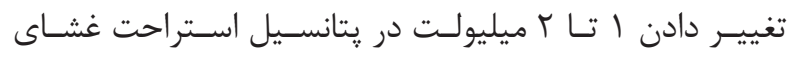

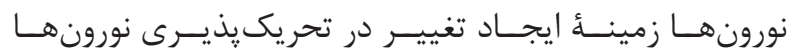

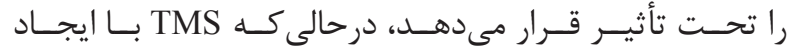

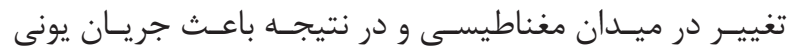

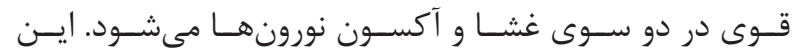

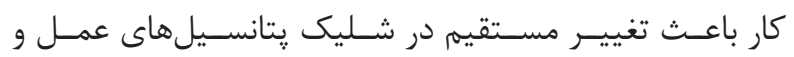

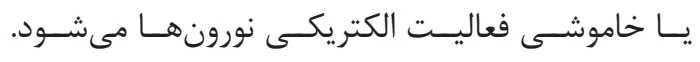

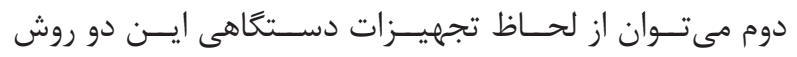

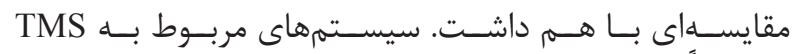

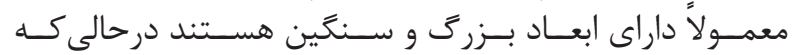

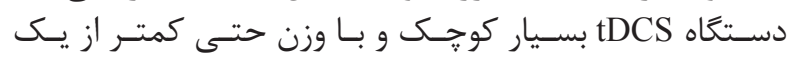

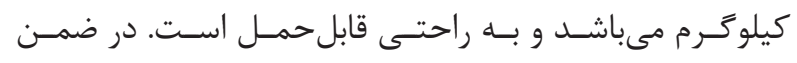

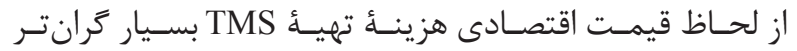

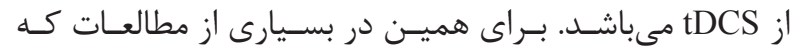

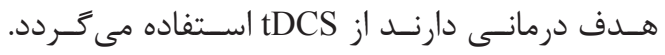

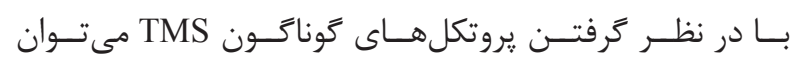

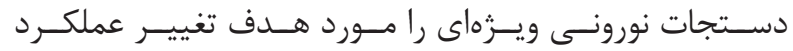

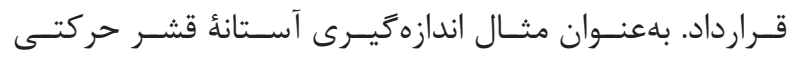

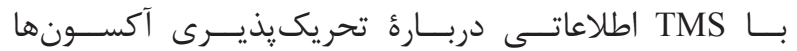

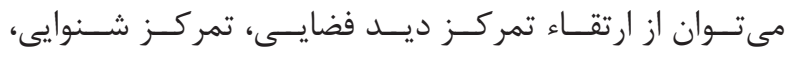

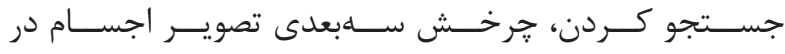

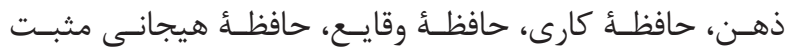

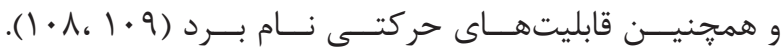

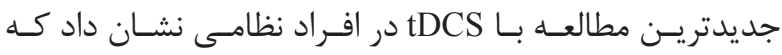

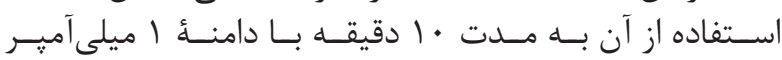

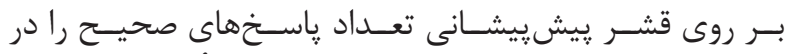

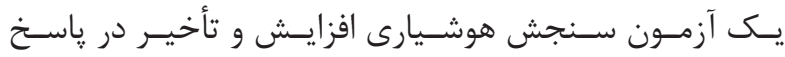

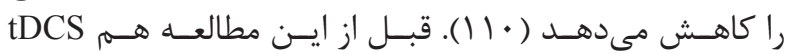

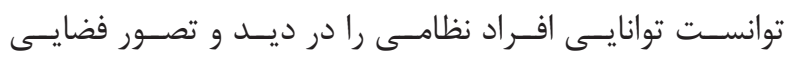

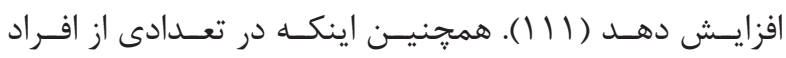

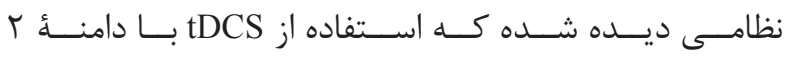

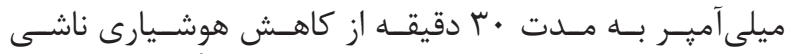

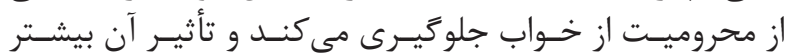

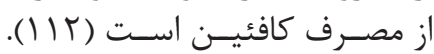

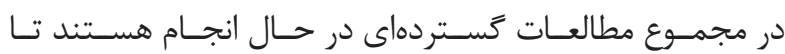

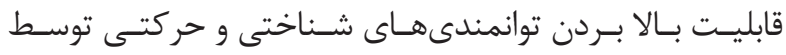

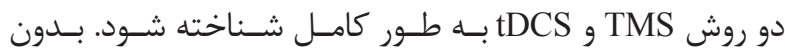

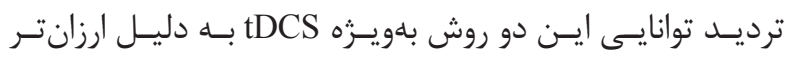

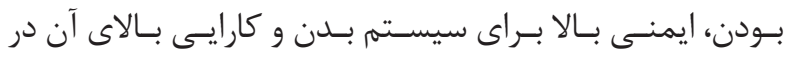

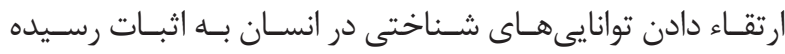

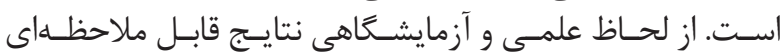

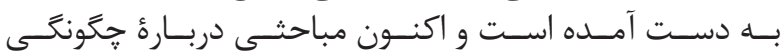

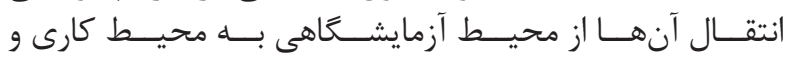

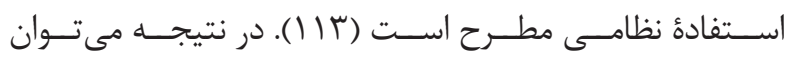

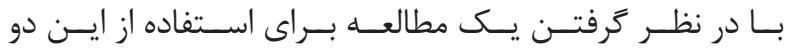

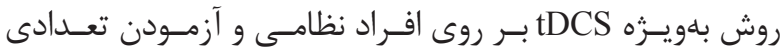

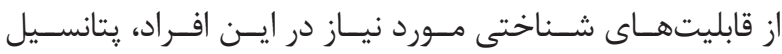

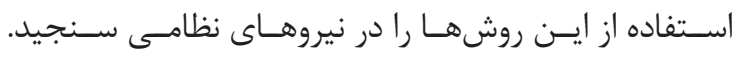

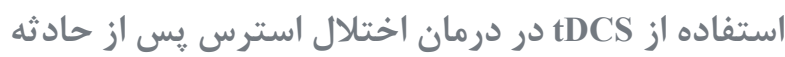

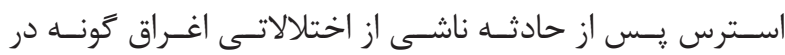

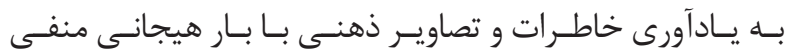

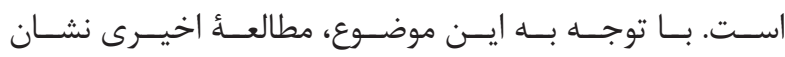

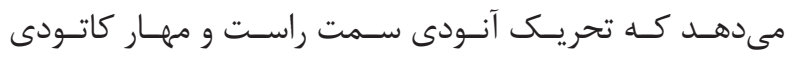
سـمت جــ

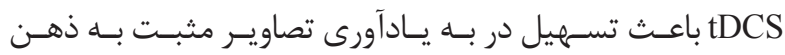

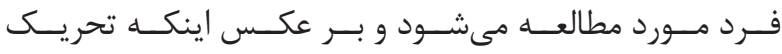

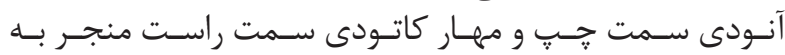

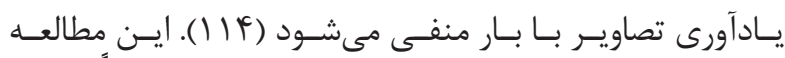

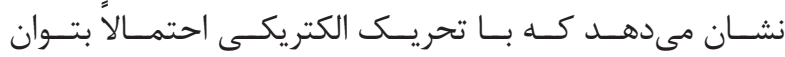

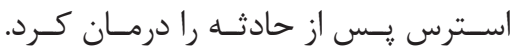

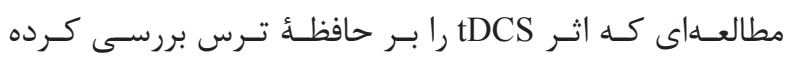

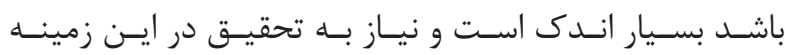

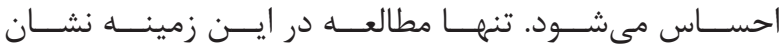

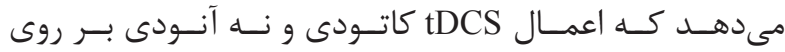


نتيجه

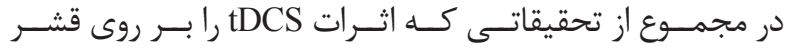

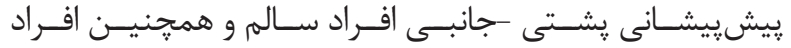

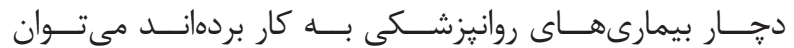

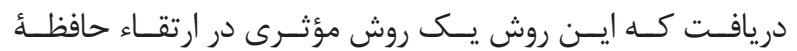

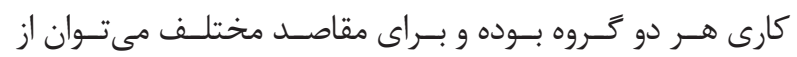

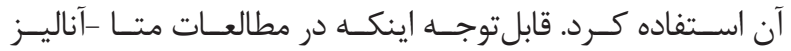

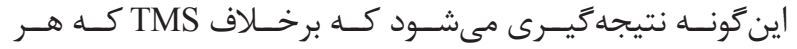

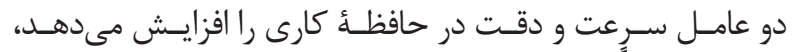

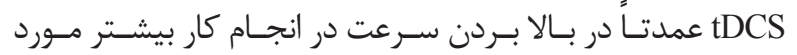

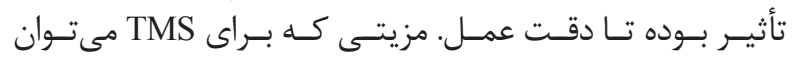

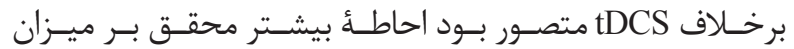

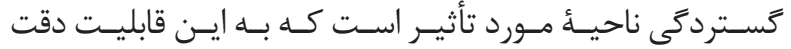

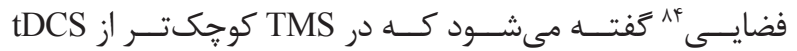

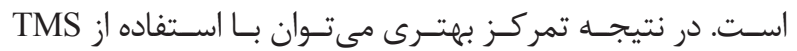

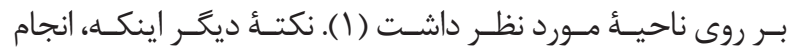

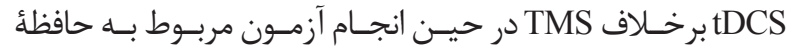

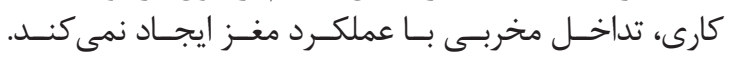

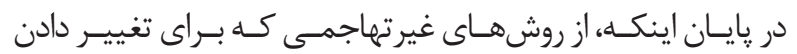

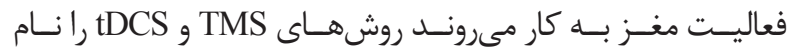

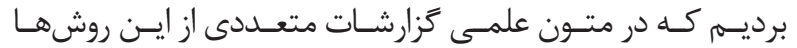

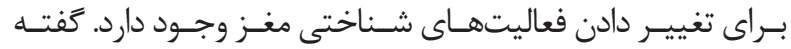

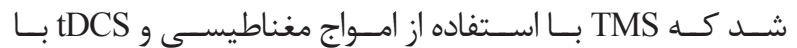

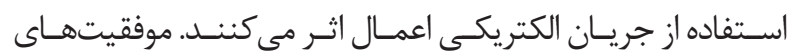

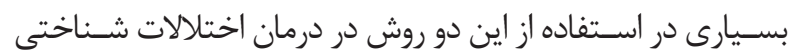

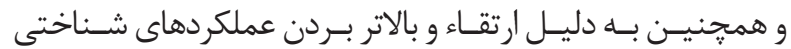

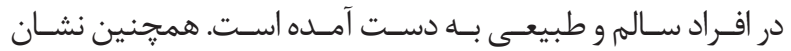

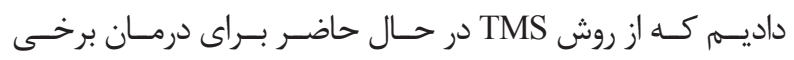

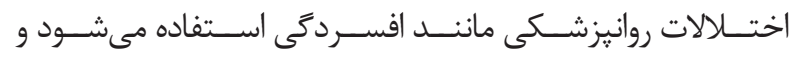

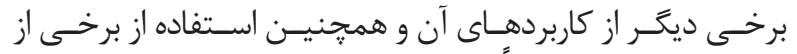

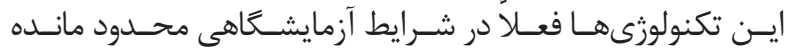

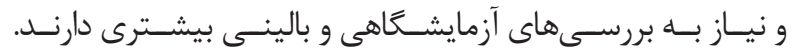

1. Dayan E, Censor N, Buch ER, Sandrini M, Cohen LG. Noninvasive brain stimulation: from physiology to network dynamics and back. Nat Neuroscience. 2013; 16: 838-44.

2. Lisanby SH, Kinnunen LH, Crupain MJ. Applications of TMS to Therapy in Psychiatry. J Clin Neurophysiol. 2002; 19(4): 344-60.

3. Aleman A. Use of repetitive transcranial magnetic stimulation for treatment in psychiatry. Clin

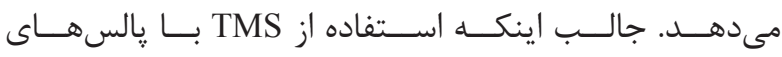

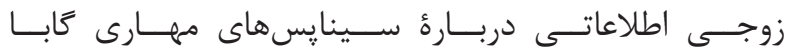

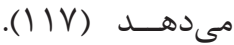

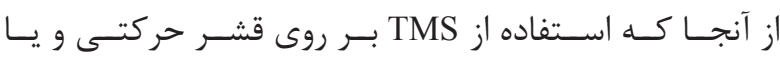

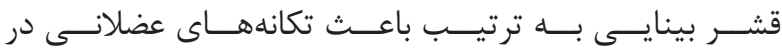

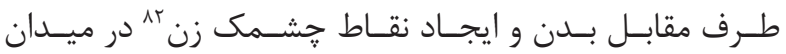

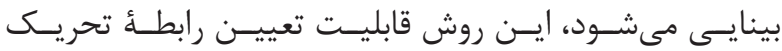

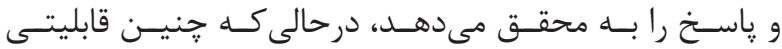

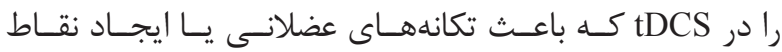

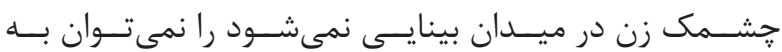

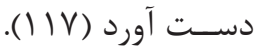

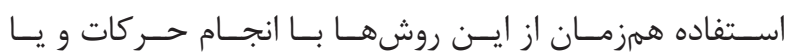

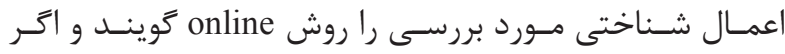

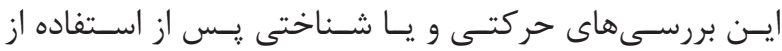

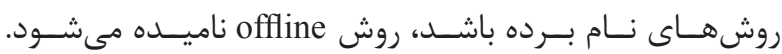

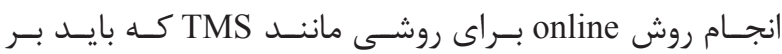

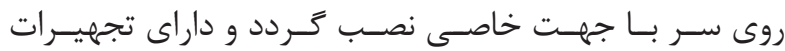

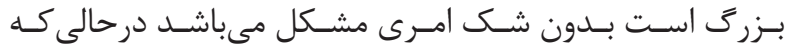

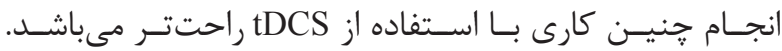

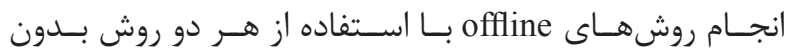
مشـكلى قابـل انجـام اسـت (I IV).

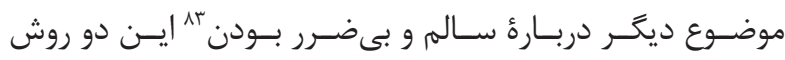

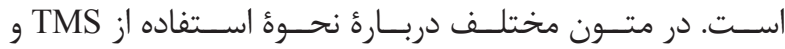

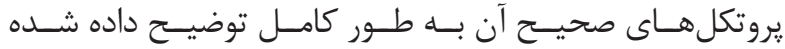

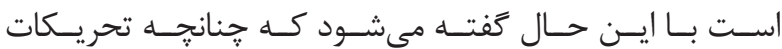

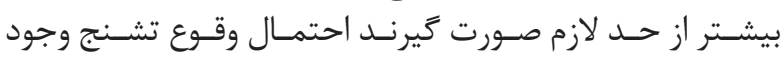

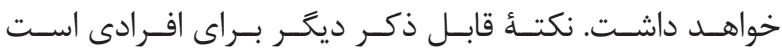

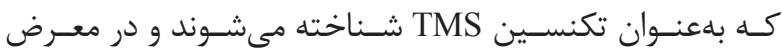

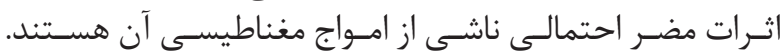

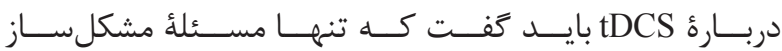

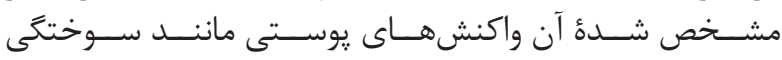

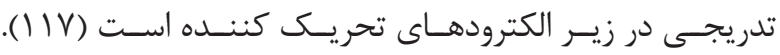

منابع

Psychopharmacol Neurosci. 2013; 11(2): 53-9.

4. Speer AM, Kimbrell TA, Wassermann EM, D Repella $\mathrm{J}$, Willis MW, Herscovitch P, et al. Opposite effects of high and low frequency rTMS on regional brain activity in depressed patients. Biol Psychiatry. 2000; 48(12): 1133-41.

5. Strafella AP, Paus T, Barrett J, Dagher A. Repetitive transcranial magnetic stimulation of the human prefrontal cortex induces dopamine release in the caudate nucleus.

\footnotetext{
${ }^{82}$ Phosphens

${ }^{83}$ Safety

${ }^{84}$ Spatial resolusion
} 
J Neurosci. 2001; 21(15): RC157.

6. Cho SS, Strafella AP. rTMS of the left dorsolateral prefrontal cortex modulates dopamine release in the ipsilateral anterior cingulate cortex and orbitofrontal cortex. PLoS One. 2009; 4(8): e6725.

7. Poldrack RA, Clark J, Pare-Blagoev EJ, Shohamy D, Creso Moyano J, Myers C, et al. Interactive memory systems in the human brain. Nature. 2001; 414(6863): 546-50.

8. Brown RM, Robertson EM. Off-line processing: reciprocal interactions between declarative and procedural memories. J Neurosci. 2007; 27(39): 10468-75.

9. Costanzi M, Saraulli D, Rossi-Arnaud C, Aceti M, Cestari V. Memory impairment induced by an interfering task is reverted by pre-frontal cortex lesions: a possible role for an inhibitory process in memory suppression in mice. Neuroscience. 2009; 158(2): 503-13.

10. Diekelmann S, Buchel C, Born J, Rasch B. Labile or stable: opposing consequences for memory when reactivated during waking and sleep. Nat Neurosci. 2011; 14: 381-6.

11. Cohen DA, Robertson EM. Preventing interference between different memory tasks. Nat Neurosci. 2011; 14(8): $953-5$.

12. Lenroot RK and Giedd JN. Brain development in children and adolescents: insights from anatomical magnetic resonance imaging. Neurosci Biobehav Rev. 2006; 30(6): 718-29.

13. Ramia L, Gironell A, Kulisevsky J, GarciaSánchez C, Berthier M, Estévez-González A. Effects of repetitive transcranial magnetic stimulation on memory subtypes: a controlled study. Neuropsychologia. 2003; 41(14): 1877-83

14. Esslinger C, Schuler N, Sauer C, Gass D, Mier D, Braun $U$, et al. Induction and quantification of prefrontal cortical network plasticity using $5 \mathrm{~Hz}$ rTMS and fMRI. Hum Brain Mapp. 2014; 35(1): 140-51.

15. Gaudeau-Bosma C, Moulier V, Allard AC, Sidhoumi D, Bouaziz N, Braha S, et al. Effect of two weeks of rTMS on brain activity in healthy subjects during an n-back task: A randomized double blind study. Brain Stimulation. 2013; 6(4): 569-75.

16. Barr MS, Farzan F, Rajji TK, Voineskos AN, Blumberger DM, Arenovich T, et al. Can repetitive magnetic stimulation improve cognition in schizophrenia? Pilot data from a randomized controlled trial. Biol Psychiatry. 2013; 73(6): 510-7.

17. Guse B, Falkai P, Gruber O, Whalley H, Gibson L, Hasan A, et al. The effect of long-term high frequency repetitive transcranial magnetic stimulation on working memory in schizophrenia and healthy controls - A randomized placebo-controlled, double-blind fMRI study. Behav Brain Res. 2013; 237: 300-7.

18. Beckers $\mathrm{G}$ and Homberg V. Impairment of visual perception and visual short term memory scanning by transcranial magnetic stimulation of occipital cortex. Exp Brain Res. 1991; 87(2): 421-32.

19. Kammer T. Masking visual stimuli by transcranial magnetic stimulation. Psychol Res. 2007; 71(6):659-66.

20. Silvanto J and Soto D. Causal evidence for subliminal percept-to-memory interference in early visual cortex. NeuroImage. 2011; 59(1):840-5.

21. Kosslyn SM, Pascual-Leone A, Felician O, Camposano S, Keenan JP, Thompson WL, et al. The role of area 17 in visual imagery: convergent evidence from PET and rTMS. Science. 1999; 284(5411): 167-70.

22. Wheeler ME, Petersen SE, Buckner RL. Memory's echo: vivid remembering reactivates sensory-specific cortex. Proc Natl Acad Sci U S A. 2000; 97(20): 111259.

23. Mottaghy FM, Sparing R, Töpper R. Enhancing picture naming with transcranial magnetic stimulation. Behav Neurol. 2006; 17(3-4):177-186.

24. Pope PA, Miall RC. Restoring cognitive functions using non-invasive brain stimulation techniques in patients with cerebellar disorders. Front Psychiatry. 2014; 5(33): doi: 10.3389/fpsyt.2014.00033.

25. Ito M. Movement and thought: identical control mechanisms by the cerebellum. Trends Neurosci. 1993; 16(11): 448-50.

26. Argyropoulos GP. Cerebellar theta-burst stimulation selectively enhances lexical associative priming. Cerebellum. 2011; 10(3): 540-50.

27. Huang YZ, Edwards MJ, Rounis E, Bhatia KP, Rothwell JC. Theta burst stimulation of the human motor cortex. Neuron. 2005; 45(2): 201-6.

28. Argyropoulos GP, Muggleton NG. Effects of Cerebellar Stimulation on Processing Semantic Associations. Cerebellum. 2013; 12(1): 83-96. 


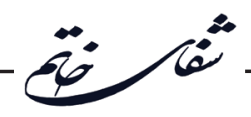

29. Schutter DJLG, Van Honk J. D’Alfonso AL, Peper JS, Panksepp J. High frequency repetitive transcranial magnetic over the medial cerebellum induces a shift in the prefrontal electroencephalography gamma spectrum: a pilot study. Neurosci Lett. 2003; 336(2): 73-6.

30. Lang P, Bradley M, Cuthbert B. International affective picture system (IAPS): Affective ratings of pictures and instruction manual. Gainesville, Fla: NIMH, Center for the Study of Emotion and Attention. 2005.

31. Schutter DJ, Van Honk J. The cerebellum in emotion regulation: a repetitive transcranial magnetic stimulation study. Cerebellum. 2009; 8(1): 28-34.

32. Balsters JH, Ramnani N. Cerebellar plasticity and the automation of first-order rules. J Neurosci. 2011; 31(6): 2305-12.

33. Fierro B, Palermo A, Puma A, Francolini M, Panetta ML, Daniele O, et al. Role of the cerebellum in time perception: a TMS study in normal subjects. J Neurol Sci. 2007; 263(1-2):107-12.

34. Lee KH, Egleston PN, Brown WH, Gregory AN, Barker AT, Woodruff PW. The role of the cerebellum in subsecond time perception: evidence from repetitive transcranial magnetic stimulation. J Cog Neurosci. 2007; 19(1): 147-57.

35. Koch G, Oliveri M, Torriero S, Salerno S, Lo Gerfo E, Caltagirone C. Repetitive TMS of cerebellum interferes with millisecond time processing. Exp Brain Res. 2007; 179(2): 291-9.

36. Pridmore S, Belmaker R. Transcranial magnetic stimulation in the treatment of psychiatric disorders. Psychiatry Clin Neurosci. 1999; 53(5): 541-8.

37. Bartrés-Faz D, Tormos JM, Junqué C, PascualLeone A.Transcranial magnetic stimulation: contribution to psychiatry and to the study of brainbehavior relationship. Actas Esp Psiquiatr. 2000; 28(2): 130-6.

38. Delgado Baquero Y, Crespo Hervás D, Cisneros S, López-Ibor Aliño JJ. Transcranial magnetic stimulation. Clinical trials in psychiatry: therapeutical use. Actas Esp Psiquiatr. 2002; 30(2): 120-8.

39. López-Ibor JJ, López-Ibor MI, Pastrana JI. Transcranial magnetic stimulation. Curr Opin Psychiatry. 2008; 21(6): 640-4.

40. Pigot M, Loo C, Sachdev P. Repetitive transcranial magnetic stimulation as treatment for anxiety disorders.
Expert Rev Neurother. 2008; 8(10): 1449-55.

41. Zwanzger P, Fallgatter AJ, Zavorotnyy M, Padberg F. Anxiolytic effects of transcranial magnetic stimulation-an alternative treatment option in anxiety disorders? J Neural Transm. 2009; 116(6): 767-75.

42. Rossi S, De Capua A, Tavanti M, Calossi S, Polizzotto NR, Mantovani A, et al. Dysfunctions of cortical excitability in drug-naïve posttraumatic stress disorder patients. Biol Psychiatry. 2009; 66(1): 54-61.

43. Rossi S, Cappa SF, Ulivelli M, De Capua A, Bartalini S, Rossini PM. rTMS for PTSD: induced merciful oblivion or elimination of abnormal hypermnesia? Behav Neurol. 2006; 17(3-4): 195-9.

44. Grisaru N, Amir M, Cohen H, Kaplan Z. Effect of transcranial magnetic stimulation in posttraumatic stress disorder: a preliminary study. Biol Psychiatry. 1998; 44(1): 52-5.

45. Rosenberg PB, Mehndiratta RB, Mehndiratta YP, Wamer A, Rosse RB, Balish M. Repetitive transcranial magnetic stimulation treatment of comorbid posttraumatic stress disorder and major depression. J Neuropsychiatry Clin Neurosci. 2002; 14(3): 270-6.

46. Cohen H, Kaplan Z, Kotler M, Kouperman I, Moisa R, Grisaru N. Repetitive transcranial magnetic stimulation of the right dorsolateral prefrontal cortex in posttraumatic stress disorder: a double-blind, placebocontrolled study. Am J Psychiatry. 2004; 161(3): 515-24.

47. Osuch EA, Benson BE, Luckenbaugh DA, Geraci M, Post RM, McCann U. Repetitive TMS combined with exposure therapy for PTSD: a preliminary study. J Anxiety Disord. 2009; 23(1): 54-9.

48. Watts BV, Landon B, Groft A, Young-Xu Y. A sham controlled study of repetitive transcranial magnetic stimulation for posttraumatic stress disorder. Brain Stimul. 2012; 5(1): 38-43.

49. Centonze D, Palmieri MG, Boffa L, Pierantozzi M, Stanzione P, Brusa L, et al. Cortical hyperexcitability in post-traumatic stress disorder secondary to minor accidental head trauma: a neurophysiologic study. J Psychiatry Neurosci. 2005; 30(2): 127-32.

50. Marin MF, Camprodon JA, Dougherty DD, Milad MR. Device-based brain stimulation to augment fear extinction: implications for PTSD treatment and beyond. Depress Anxiety. 2014; 31(4): 269-78.

51. Karsen EF, Watts BV, Holtzheimer PE. Review 
of the effectiveness of transcranial magnetic stimulation for post-traumaticstress disorder. Brain Stimul. 2014; 7(2):151-7.

52.BerlimMT, VanDenEyndeF. Repetitive transcranial magnetic stimulation over the dorsolateral prefrontal cortex for treating posttraumatic stress disorder: an exploratory meta-analysis of randomized, double-blind and sham-controlled trials. Can J Psychiatry. 2014; 59(9): 487-96.

53. Boggio PS, Rocha M, Oliveira MO, Fecteau S, Cohen RB, Campanhã $\mathrm{C}$, et al. Noninvasive brain stimulation with high-frequency and low-intensity repetitive transcranial magnetic stimulation treatment for posttraumatic stress disorder. J Clin Psychiatry. 2010; 71(8): 992-9.

54. Isserles M, Shalev AY, Roth Y, Peri T, Kutz I, Zlotnick E, et al. Effectiveness of deep transcranial magnetic stimulation combined with a brief exposure procedure in post-traumatic stress disorder--a pilot study. Brain Stimul. 2013; 6(3): 377-83.

55. Nam DH, Pae CU, Chae JH. Low-frequency, repetitive transcranial magnetic stimulation for the treatment of patients with posttraumatic stress disorder: a double-blind, sham-controlled Study. Clin Psychopharmacol Neurosci. 2013; 11(2): 96-102.

56. Tillman GD, Kimbrell TA, Calley CS, Kraut MA, Freeman TW, Hart J Jr. Repetitive transcranial magnetic stimulation and threat memory: selective reduction of combat threat memory p300 response after right frontal-lobe stimulation. J Neuropsychiatry Clin Neurosci. 2011; 23(1): 40-7.

57. Oznur T, Akarsu S, Celik C, Bolu A, Ozdemir $\mathrm{B}$, Akcay $\mathrm{BD}$, et al. Is transcranial magnetic stimulation effective in treatment-resistant combat related posttraumatic stress disorder? Neurosci (Riyadh). 2014; 19(1): 29-32.

58. Miranda PC, Lomarev M, Hallett M. Modeling the current distribution during transcranial direct current stimulation. Clin Neurophysiol. 2006; 117(7): 1623-9.

59. Nitsche MA, Fricke K, Henschke U, Schlitterlau A, Liebetanz, D, Lang $\mathrm{N}$, et al. Pharmacological modulation of cortical excitability shifts induced by transcranial direct current stimulation in humans. J Physiol. 2003; 553(1): 293-301.

60. Nitsche MA, Doemkes S, Karaköse T, Antal A, Liebetanz D, Lang N, et al. Shaping the effects of transcranial direct current stimulation of the human motor cortex. J Neurophysiol. 2007; 97(4): 3109-17.

61. Nitsche MA, Niehaus L, Hoffmann KT, Hengst S, Liebetanz D, Paulus W, et al. MRI study of human brain exposed to weak direct current stimulation of the frontal cortex. Clin Neurophysiol. 2004; 115(10): 2419-23.

62. Ardolino G, Bossi B, Barbieri S, Priori A. Nonsynaptic mechanisms underlie the after-effects of cathodal transcutaneous direct current stimulation of the human brain. J Physiol. 2005; 568(2): 653-63.

63. Nitsche MA, Seeber A, Frommann K, Klein CC, Rochford C, Nitsche MS, et al. Modulating parameters of excitability during and after transcranial direct current stimulation of the human motor cortex. J Physiol. 2005; 568(1): 291-303.

64. Fregni F, Boggio PS, Nitsche M, Bermpohl F, Antal A, Feredoes E, et al. Anodal transcranial direct current stimulation of prefrontal cortex enhances working memory. Exp Brain Res. 2005; 166(1): 23-30.

65. Keeser D, Padberg F, Reisinger E, Pogarell O, Kirsch $\mathrm{V}$, Palm U, et al. Prefrontal direct current stimulation modulates resting EEG and event-related potentials in healthy subjects: a standardized low resolution tomography (sLORETA) study. Neuroimage. 2013; 55(2): 644-57.

66. Ohn SH, Park CI, Yoo WK, Ko MH, Choi KP, Kim $\mathrm{GM}$, et al. Time-dependent effect of transcranial direct current stimulation on the enhancement of working memory. Neuroreport. 2008; 19(1): 43-7.

67. Mulquiney PG, Hoy KE, Daskalakis ZJ, Fitzgerald PB. Improving working memory: exploring the effect of transcranial random noise stimulation and transcranial direct current stimulation on the dorsolateral prefrontal cortex. Clin Neurophysiol. 2011; 122(12): 2384-9.

68. Mylius V, Jung M, Menzler K, Haag A, Khader PH, Oertel WH, et al. Effects of transcranial direct current stimulation on pain perception and working memory. Europ J Pain. 2012; 16(7): 974-82.

69. Teo F, Hoy KE, Daskalakis ZJ, Fitzgerald PB. Investigating the role of current strength in tDCS modulation of working memory performance in healthy controls. Front Psychiatry. 2011; 2: 45. doi: 10.3389/ fpsyt.2011.00045.

70. Lally N, Nord CL, Walsh V, Roiser JP. Does excitatory fronto-extracerebral tDCS lead to improved working memory performance? F1000Research. 2013; 2: 219. doi: 10.12688/f1000research.2-219.v2. 
71. Andrews SC, Hoy KE, Enticott PG, Daskalakis ZJ, Fitzgerald PB. Improving working memory: the effect of combining cognitive activity and anodal transcranial direct current stimulation to the left dorsolateral prefrontal cortex. Brain Stimul. 2011; 4(2): 84-9.

72. Berryhill ME, Jones KT. tDCS selectively improves working memory in older adults with more education. Neurosci Lett. 2012; 521(2): 148-51.

73. Kincses TZ, Antal A, Nitsche MA, Bartfai O, Paulus W. Facilitation of probabilistic classification learning by transcranial direct current stimulation of the prefrontal cortex in the human. Neuropsychologia. 2004; 42(1): 113-7.

74. Manenti R, Brambilla M, Petesi M, Ferrari C, Cotelli M. Enhancing verbal episodic memory in older and young subjects after non-invasive brain stimulation. Front Aging Neurosci. 2013; 5: 49. doi: 10.3389/fnagi.2013.00049.

75. Zwissler B, Sperber C, Aigeldinger S, Schindler S, Kissler J, Plewnia C. Shaping memory accuracy by left prefrontal transcranial direct current stimulation. J Neurosci. 2014; 34(11): 4022-6.

76. Brunoni AR, Valiengo L, Baccaro A, Zanão TA, de Oliveira JF, Goulart A, et al. The sertraline vs. electrical current therapy for treating depression clinical study: results from a factorial, randomized, controlled trial. JAMA Psychiatry. 2013; 70(4): 383-91.

77. BrunoniAR, Zanao TA, Ferrucci R, PrioriA, Valiengo L, De Oliveira JF, et al. Bifrontal tDCS prevents implicit learning acquisition in antidepressant-free patients with major depressive disorder. Prog Neuropsychopharmacol Biol Psychiatry. 2013; 43: 146-50.

78. Fregni F, Boggio PS, Nitsche MA, Marcolin MA, Rigonatti SP, Pascual-Leone A. Treatment of major depression with transcranial direct current stimulation. Bipolar Disord. 2006a; 8(2): 203-4.

79. Fregni F, Boggio PS, Nitsche MA, Rigonatti SP, Pascual-Leone A. Cognitive effects of repeated sessions of transcranial direct current stimulation in patients with depression. Depress Anxiety. 2006b; 23(8): 482-4.

80. Oliveira JF, Zanao TA, Valiengo L, Lotufo PA, Bensenor IM, Fregni F, et al. Acute working memory improvement after tDCS in antidepressant-free patients with major depressive disorder. Neurosci Lett. 2013; 537: $60-4$

81. Wolkenstein L, Plewnia C. Amelioration of cognitive control in depression by transcranial direct current stimulation. Biol Psychiatry. 2013; 73(7): 646-51.

82. Vercammen A, Rushby JA, Loo C, Short B, Weickert CS, Weickert TW. Transcranial direct current stimulation influences probabilistic association learning in schizophrenia. Schizophr Res. 2011; 131(1-3): 198-205.

83. Hoy KE, Arnold SL, Emonson MR, Daskalakis ZJ, Fitzgerald PB. An investigation into the effects of tDCS dose on cognitive performance over time in patients with schizophrenia. Schizophr Res. 2014; 155(1-3): 96-100.

84. Goder R, Baier PC, Beith B, Baecker C, SeeckHirschner M, Junghanns K, et al. Effects of transcranial direct current stimulation during sleep on memory performance in patients with schizophrenia. Schizophr Res. 2013; 144(1-3): 153-4.

85. Boggio PS, Khoury LP, Martins DC, Martins OE, DeMacedo EC, Fregni F. Temporal cortex direct current stimulation enhances performance on a visual recognition memory task in Alzheimer disease. J Neurol Neurosurg Psychiatr. 2009; 80(4): 444-7.

86. Cotelli M, Manenti R, Brambilla M, Petesi M, Rosini S, Ferrari C, et al. Anodal tDCS during face-name associations memory training in Alzheimer's patients. Front Aging Neurosci. 2014; 6: 38. doi: 10.3389/ fnagi.2014.00038.

87. Boggio PS, Ferrucci R, Rigonatti SP, Covre P, Nitsche M, Pascual-Leone A, et al. Effects of transcranial direct current stimulation on working memory in patients with Parkinson's disease. J Neurol Sci. 2006; 249(1): 31-8.

88. Pereira JB, Junque C, Bartres-Faz D, Marti MJ, SalaLlonch R, Compta Y, et al. Modulation of verbal fluency networks by transcranial direct current stimulation (tDCS) in Parkinson's disease. Brain Stimul. 2013; 6(1): $16-24$.

89. Kang EK, Baek MJ, Kim S, Paik NJ. Non-invasive cortical stimulation improves post-stroke attention decline. Restor Neurol Neurosci. 2009; 27(6): 645-50.

90. Jo JM, Kim YH, Ko MH, Ohn SH, Joen B, Lee KH. Enhancing the working memory of stroke patients using tDCS. Am J Phys Med Rehabil. 2009; 88(5): 404-9.

91. Park SH, Koh EJ, Choi HY, Ko MH. A double-blind, sham- controlled, pilot study to assess the effects of the concomitant use of transcranial direct current stimulation with the computer assisted cognitive rehabilitation to the prefrontal cortex on cognitive functions in patients with stroke. J Korean Neurosurg Soc. 2013; 54(6): 484-8. 
92. De Smet HJ, Paquier P, Verhoeven J, Marien P. The cerebellum: its role in language and related cognitive and affective functions. Brain Lang. 2013; 127(3): 334-42.

93. Stoodley CJ. The cerebellum and cognition: evidence from functional imaging studies. Cerebellum. 2012; 11(2): 352-65.

94. Baumann O, Mattingley JB. Functional topography of primary emotion processing in the human cerebellum. NeuroImage. 2012; 61(4): 805-11.

95. Minks E, Kopickova M, Marecek R, Streitova H, Bares M. Transcranial magnetic stimulation of the cerebellum. Biomed Pap Med Fac Univ Palacky Olomouc Czech Repub. 2010; 154(2): 133-9.

96. Ferrucci R, Marceglia S, Vergari M, Cogiamanian F, Mrakic-Sposta S, Mameli F, et al. Cerebellar transcranial direct current stimulation impairs the practice-dependent proficiency increase in working memory. J Cogn Neurosci. 2008; 20(9): 1687-97.

97. Priori A. Brain polarization in humans: a reappraisal of an old tool for prolonged non-invasive modulation of brain excitability. Clin Neurophysiol. 2003; 114(4): 589-95.

98. Boehringer A, Macher K, Dukart J, Villringer A, Pleger B. Cerebellar transcranial direct current stimulation modulates verbal working memory. Brain Stimul. 2013; 6(4): 649-53.

99. Pope PA, Miall RC. Task-specific facilitation of cognition by cathodal transcranial direct current stimulation of the cerebellum. Brain Stimul. 2012; 5(2): 84-94.

100. O'Halloran CJ, Kinsella GJ, Storey E. The cerebellum and neuropsychological functioning: a critical review. J Clin Exp Neuropsychol. 2012; 34(1): 35-56.

101. Ferrucci R, Brunoni AR, Parazzini M, Vergari M, Rossi E, Fumagalli M, et al. Modulating human procedural learning by cerebellar transcranial direct current stimulation. Cerebellum. 2013; 12(4): 485-92.

102. Rogers TD, Dickson PE, Heck DH, Goldowitz, D, Mittleman G, Blaha CD. Connecting the dots of the cerebro-cerebellar role in cognitive function: neuronal pathways for cerebellar modulation of dopamine release in the prefrontal cortex. Synapse. 2012; 65(11): 1204-12.

103. Sehm B, Ragert P. Why non-invasive brain stimulation should not be used in military and security services. Front Hum Neurosci. 2013; 7: 553. doi: 10.3389/fnhum.2013.00553.

104. Clark VP, Coffman BA, Mayer AR, Weisend MP, Lane TD, Calhoun VD, et al. TDCS guided using fMRI significantly accelerates learning to identify concealed objects. Neuroimage. 2012; 59(1): 117-28.

105. Sehm B, Schaefer A, Kipping J, Margulies D, Conde $\mathrm{V}$, Taubert $\mathrm{M}$, et al. Dynamic modulation of intrinsic functional connectivity by transcranial direct current stimulation. J Neurophysiol. 2012; 108(12): 3253-63.

106. Pavlidou A, Edwards, M, Lange J, Schnitzler A, Bell J. Anodal stimulation of premotor cortex facilitates the recognition of different forms of movements. In Proceedings of the Neuro Visionen 8 Conference, 95, (Aachen). 2012.

107. Falcone B, Coffman BA, Clark VP, Parasuraman R. Transcranial direct current stimulation enhances perceptual sensitivity and 24-hour retention in a complex threat detection task. PLoS One. 2012; 7(4):e34993. doi: 10.1371/journal.pone.0034993.

108. McKinley RA, Bridges N, Walters CM, Nelson J. Modulating the brain at work using noninvasive transcranialstimulation. Neuroimage.2012;59(1):129-37.

109. Parasuraman R, McKinley RA. Using noninvasive brain stimulation to accelerate learning and enhance human performance. Hum Factors. 2014; 56: 1-9.

110. Nelson JT, McKinley RA, Golob EJ, Warm JS, Parasuraman R. Enhancing vigilance in operators with prefrontal cortex transcranial direct current stimulation (tDCS). Neuroimage. 2014; 85: 909-17.

111. McKinley RA, McIntire L, Bridges N, Goodyear C, Weisend MP. Acceleration of image analyst training with transcranial direct current stimulation. Behav Neurosci. 2013; 127(6): 936-46.

112. McIntire LK, McKinley RA, Goodyear C, Nelson J. A comparison of the effects of transcranial direct current stimulation and caffeine on vigilance and cognitive performance during extended wakefulness. Brain Stimul. 2014; 7(4): 499-507.

113. Nelson JT, Tepe V. Neuromodulation research and application in the u.s. department of defense. Brain Stimul. 2015; 8(2): 247-52.

114. Penolazzi B, Di Domenico A, Marzoli D, Mammarella N, Fairfield B, Franciotti R, et al. Effects 


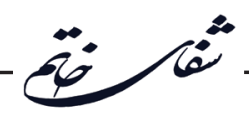

of transcranial direct current stimulation on episodic memory related to emotional visual stimuli. PloS One. 2010; 5(5): e10623. doi: 10.1371/journal. pone. 0010623 .

115. Asthana M, Nueckel K, Mühlberger A, Neueder D, Polak T, Domschke K. et al. Effects of transcranial direct current stimulation on consolidation of fear memory. Front Psychiatry. 2013; 4: 107. doi: 10.3389/ fpsyt.2013.00107.
116. Saunders N, Downham R, Turman B, Kropotov J, Clark R, Yumash R, et al. Working memory training with tDCS improves behavioral and neurophysiological symptoms in pilot group with posttraumatic stress disorder (PTSD) and with poor working memory. Neurocase. 2015; 21(3): 271-8.

117. Priori A, Hallett M, Rothwell J. Repetitive transcranial magnetic stimulation or transcranial direct current stimulation? Brain Stimul. 2009; 2(4): 241-5. 